\title{
The Tevatron Tune Tracker PLL - Theory, Implementation and Measurements
}

\author{
Cheng-Yang Tan \\ Accelerator Division/Tevatron
}

\begin{abstract}
The Tevatron tune tracker is based on the idea that the transverse phase response of the beam can be measured quickly and accurately enough to allow us to track the betatron tune with a phase locked loop (PLL). The goal of this paper is to show the progress of the PLL project at Fermilab. We will divide this paper into three parts: theory, implementation and measurements. In the theory section, we will use a simple linear model to show that our design will track the betatron tune under conditions that occur in the Tevatron. In the implementation section we will break down and examine each part of the PLL and in some cases calculate the actual PLL parameters used in our system from beam measurements. And finally in the measurements section we will show the results of the PLL performance.
\end{abstract}




\section{INTRODUCTION}

The Tevatron tune tracker is a Run II project whose goal is to be able to track the proton tune up the ramp and through the squeeze for every store. The holy grail is to eventually build a tune and chromaticity feedback system around the tune tracker in order to control these two critical parameters up the ramp. The idea behind the tune tracker is that the phase response of the beam can be quickly measured and that the phase null of the response is at the peak of the resonance. By following this null, any motion of the resonance peak can be tracked. Tracking with this method is called the PLL (phase locked loop) method. This method is also of interest for the LHC (Large Hadron Collider) and so part of the funding of this project also comes from LARP (LHC Accelerator Research Program). As part of the LARP collaboration, BNL (Brookhaven National Laboratory) has also been tasked with looking at tune tracking with a PLL as a possible method of doing tune feedback up the ramp at the LHC.

Tune tracking with a PLL is not new. BNL ${ }^{1,2,3}$ has had many years of experience with this method. What Fermilab brings to the table is that we have a working machine - the Tevatron — that accelerates bunches of protons (and anti-protons) to higher energy than RHIC (Relativistic Heavy Ion Collider). This presents an opportunity to test the PLL on beam that is at a different regime than at RHIC.

The PLL which we designed for the Tevatron has the capability of doing multitone (three tones) PLL and pulsed PLL. Due to the compressed time frame of this project, we have only built a PLL for measuring the horizontal tune and not all the parts of the design have been implemented yet. Our main goal at the time was to build as much of the system as necessary to show that the tune tracker will work when in the continuously or pulsed beam excitation modes. As of this writing, although this project has been active for about 10 months (September 2003 to end of July 2004), a lot has already been done and the 
results will be presented here.

This paper is divided into four sections and two appendices. The four sections are Theory, Implementation, Measurements and Epilogue. In the Theory section, we will show the derivation of a linear theory of the PLL so that we can show that our design will lock to the betatron tune in the cases of a phase jump, frequency jump and frequency ramp. In the Implementation section, we will show how the PLL parameters are found from actual beam measurements. We will also discuss the software portion of the PLL, kicker feedback, Schottky detector, automatic gain control and phase compenstaton. In the Measurements section, we will show the bench measurements of the PLL electronic characteristics as well as the tracking of actual beam using both the continuous and pulsed excitation methods. The Epilogue section contains future plans and conclusion from the work done so far.

Two other parts of this project have helped speed up our development of the system considerably:

(i) The complete description of the PLL in software. This has allowed us to simulate the behaviour of the PLL under different excitation and beam conditions which has helped us understand and debug the actual hardware. This software model of the PLL will not be discussed in this paper.

(ii) The "fake beam frequency response" circuit which has allowed us to test the PLL on the bench before using any precious Tevatron study time. The theory of this circuit will be discussed in Appendix $I$. 
Theory 


\section{THEORY}

The goal of this section is to analyze the PLL performance with a linear mathematical model and to show that our final implementation will work. In order to get analytic solutions, we will make two beam phase response approximations:

(i) The beam response is a damped simple harmonic oscillator. We will ignore synchrotron tune lines and just look at the betatron tune line only. This is a reasonable approximation because the goal of the PLL is to lock to the betatron tune.

(ii) We will linearize the phase response close to resonance. We can do this if the PLL excitation frequency never moves too far away from resonance.

After we have the beam phase response approximation, we will calculate the transfer function $\mathcal{H}$ which relates the phase of the excitation to the phase of the beam resonance. This is exactly what our PLL does: if the PLL is designed correctly then when the beam resonance moves (like ramping etc.), the transfer function $\mathcal{H}$ will move the excitation phase to follow this change.

In order to characterize the steady state properties of the PLL, we will calculate the error transfer function $\mathcal{H}_{e}$ of the PLL. The error phase is defined to be the phase difference between the excitation phase and the phase of the beam resonance. We will show that for the cases when the beam resonance does a phase jump, frequency jump and frequency ramp, the PLL will eventually lock to the beam resonance.

In this paper we will write the time domain phases in capitals $\Theta(t)$ and the $s$-domain phases in lower case $\theta(s)$.

Finally, at the end of this section, we will also show the theory behind the pulsed PLL. The technique of pulse exciting the beam will enable us to always lock to the centre of the tune line rather than to some arbitrary synchrotron line. 


\section{LINEARIZATION OF THE BEAM PHASE RESPONSE}

Let us assume that the beam frequency response is that of a simple harmonic oscillator (sho). The magnitude and phase of the sho is shown in Figure 1(a). Next we can linearize the phase response of the sho if we are close to $\omega_{0}$ like in Figure 1(b). This means that we can approximate the phase around $\omega_{0}$ as a straight line with slope $-K$. It is well known that at resonance $\omega_{0}=\dot{\Theta}_{0}$ the phase shift should be $-90^{\circ}$, however, it is easier for our calculations if we make the phase shift zero at resonance by adding a $90^{\circ}$ phase shifter to the beam. See Figure 1(c).

For example, if the excitation sine wave before going into the beam is at phase $\Theta_{\text {in }}$, then after the beam, the output phase $\Theta_{\text {out }}$ is related to $\Theta_{\text {in }}$ by a linear phase shift, i.e.

$$
\Theta_{\text {out }}=\Theta_{\text {in }}-K\left(\dot{\Theta}_{\text {in }}-\dot{\Theta}_{0}\right)
$$

Clearly from (1), the excitation sine wave will not be phase shifted when $\dot{\Theta}_{\text {in }}=\dot{\Theta}_{0}=\omega_{0}$. This is exactly when phase locking occurs.

The Laplace transform of (1) is

$$
\begin{aligned}
\theta_{\text {out }} & =\theta_{\text {in }}-K s\left(\theta_{\text {in }}-\theta_{0}\right)+K\left(\Theta_{\text {in }}(0)-\Theta_{0}(0)\right) \\
& =\theta_{\text {in }}-K s\left(\theta_{\text {in }}-\theta_{0}\right) \\
& =(1-K s) \theta_{\text {in }}+K s \theta_{0}
\end{aligned}
$$

where we have selected the initial condition $\Theta_{\text {in }}(0)=\Theta_{0}(0)=0$. This choice means that the excitation does not start until $t>0$. 

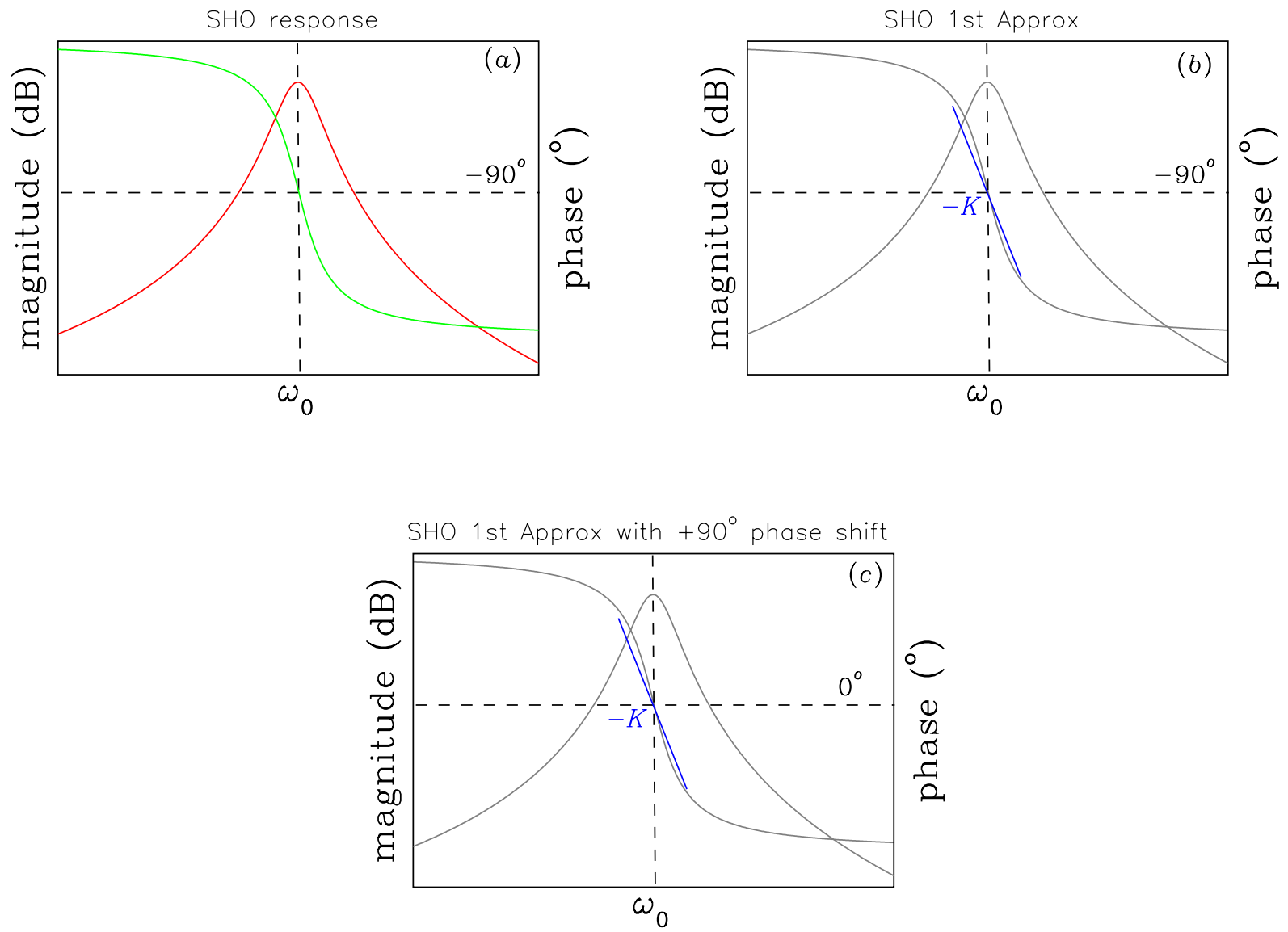

Figure 1 The stages of how we obtain the beam phase response approximation.

\section{PLL TRANSFER FUNCTION}

Referring to Figure 2, the frequency response $\mathcal{H}$ which relates $\theta_{0}$ to $\theta_{2}$ is

$$
\theta_{2}=\mathcal{H} \theta_{0}
$$

The motivation for finding this relationship rather than others is because in real life when the resonant frequency $\dot{\Theta}_{0}$ of the beam response moves we want to know how well the excitation $\dot{\Theta}_{2}$ of the PLL tracks it. 


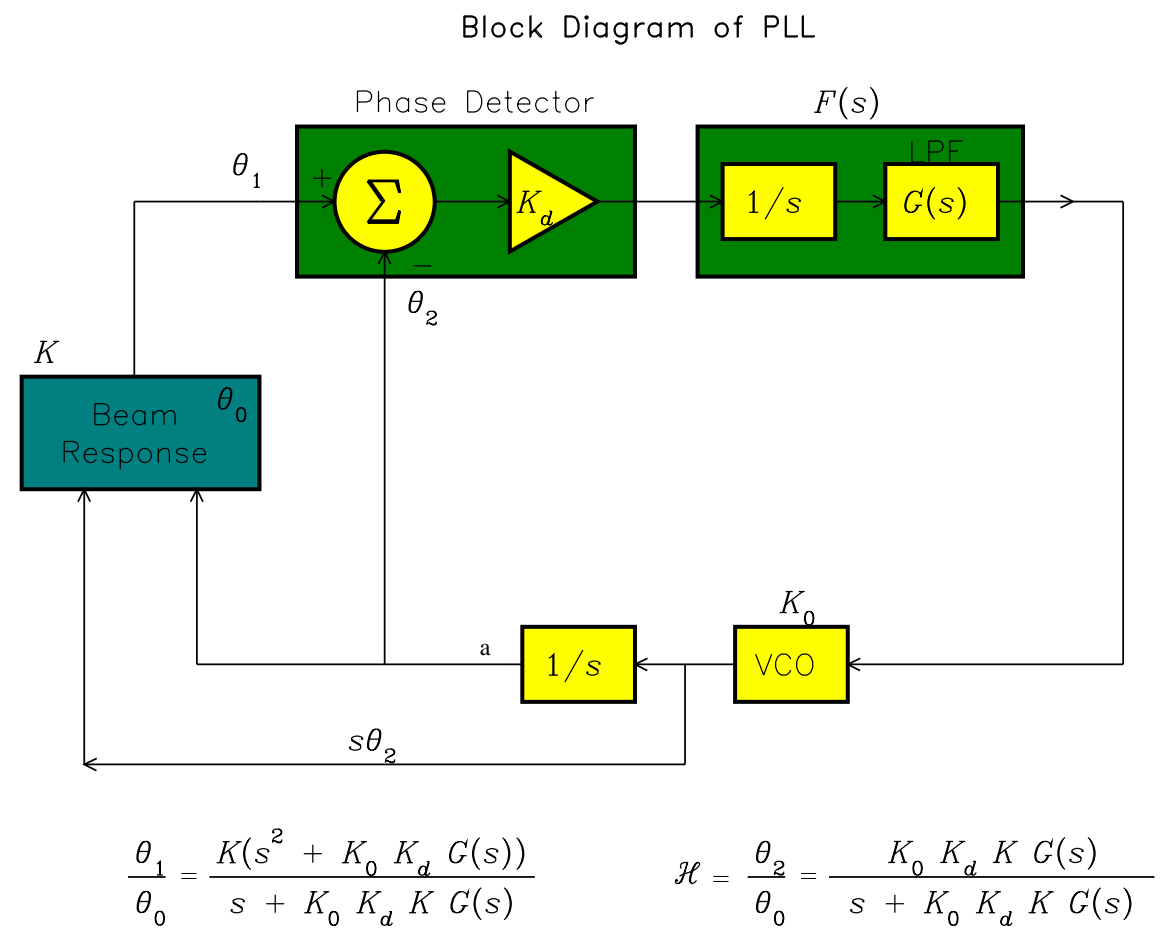

Figure 2 This is the block diagram of the PLL.

At point a of Figure 2, we have

$$
\left.\begin{array}{rl}
\theta_{2} & =\frac{K_{0} K_{d}}{s} F(s)\left(\theta_{1}-\theta_{2}\right) \\
\theta_{2} & =\frac{K_{0} K_{d} F(s) \theta_{1}}{s+K_{0} K_{d} F(s)}
\end{array}\right\}
$$

The output $\theta_{1}$ after going through the beam using the approximate beam response (2) is

$$
\theta_{1}=(1-K s) \theta_{2}+K s \theta_{0}
$$

By substituting $\theta_{1}$ from (5) into (4), we have

$$
\frac{\theta_{2}}{\theta_{0}}=\frac{K_{0} K_{d} K F(s)}{1+K_{0} K_{d} K F(s)} \equiv \mathcal{H}(s)
$$

which gives us the required relationship. Substituting $F(s)=G(s) / s$ into (6), we get

$$
\mathcal{H}(s)=\frac{K_{0} K_{d} K G(s)}{s+K_{0} K_{d} K G(s)}
$$


Note that the integrator $1 / s$ is important for the PLL to track the tune in certain conditions. This will be discussed in section No Integrator.

For completeness, a similar expression which relates $\theta_{1}$ to $\theta_{0}$ is

$$
\frac{\theta_{1}}{\theta_{0}}=\frac{K\left(s^{2}+K_{0} K_{d} G(s)\right)}{s+K_{0} K_{d} K G(s)}
$$

\section{Normalized Form}

Let us choose $G(s)$ to be a passive lag filter

$$
G(s)=\frac{g\left(1+s \tau_{2}\right)}{1+s\left(\tau_{1}+\tau_{2}\right)}
$$

where $\tau_{1}=R_{1} C, \tau_{2}=R_{2} C$ and $g$ is the gain of the filter with dimensions of $1 /$ time $(g$ has the dimensions which ensure that $F(s)$ is dimensionless). The passive lag filter circuit and Bode plot is shown in Figure 3.
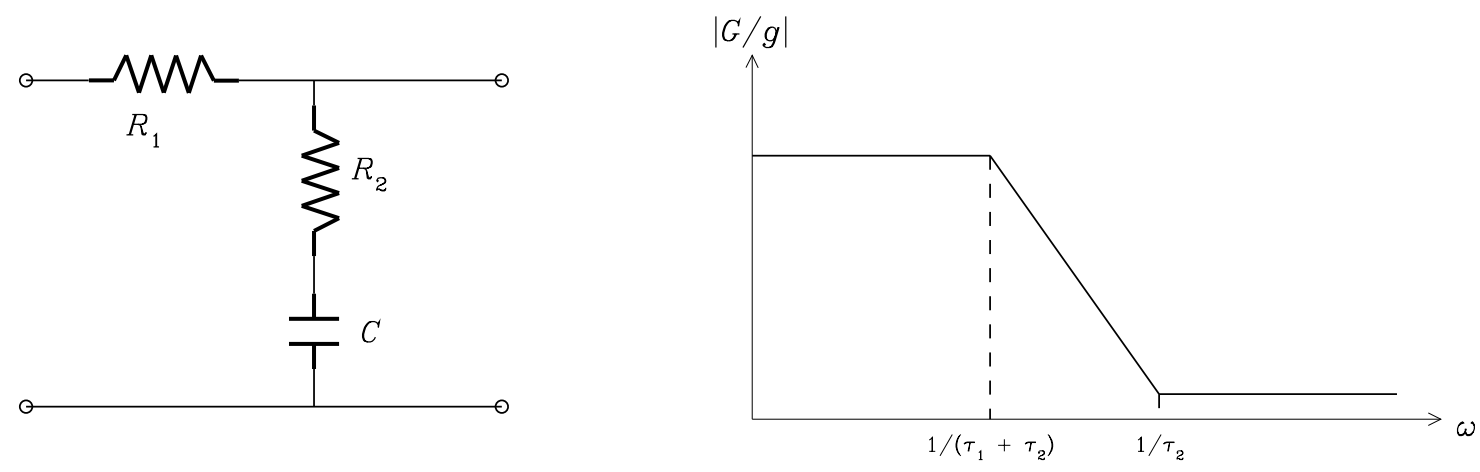

Figure 3 The passive lag filter circuit and its Bode diagram.

Then $\mathcal{H}(s)$ becomes

$$
\mathcal{H}(s)=\frac{\frac{g K_{0} K_{d} K \tau_{2} s}{\tau_{1}+\tau_{2}}+\frac{g K_{0} K_{d} K}{\tau_{1}+\tau_{2}}}{s^{2}+\frac{\left(1+g K_{0} K_{d} K \tau_{2}\right) s}{\tau_{1}+\tau_{2}}+\frac{g K_{0} K_{d} K}{\tau_{1}+\tau_{2}}}
$$


We can identify the usual quantities

$$
\left.\begin{array}{rl}
\omega_{n} & =\sqrt{\frac{g K_{0} K_{d} K}{\tau_{1}+\tau_{2}}} \quad \Rightarrow \omega_{n}^{2}=\frac{g K_{0} K_{d} K}{\tau_{1}+\tau_{2}} \\
\zeta & =\frac{1+g K_{0} K_{d} K \tau_{2}}{2\left(\tau_{1}+\tau_{2}\right) \omega_{n}} \quad \Rightarrow \zeta=\frac{\omega_{n}}{2}\left(\tau_{2}+\frac{1}{g K_{0} K_{d} K}\right)
\end{array}\right\}
$$

where $\omega_{n}$ is the natural frequency and $\zeta$ is the damping factor. So $\mathcal{H}$ written with these quantities is

$$
\begin{aligned}
\mathcal{H}(s) & =\frac{s \omega_{n}\left(2 \zeta-\frac{\omega_{n}}{g K_{0} K_{d} K}\right)+\omega_{n}^{2}}{s^{2}+2 s \zeta \omega_{n}+\omega_{n}^{2}} \\
& \approx \frac{2 \zeta \omega_{n} s+\omega_{n}^{2}}{s^{2}+2 s \zeta \omega_{n}+\omega_{n}^{2}} \quad \text { if } \quad \omega_{n} / g K_{0} K_{d} K \ll 2 \zeta
\end{aligned}
$$

(A quick sanity check shows that when $s \rightarrow 0 \mathcal{H} \rightarrow 1$ ). The Bode plot of $\mathcal{H}(s)$ for different $\zeta$ values is shown in Figure 4.

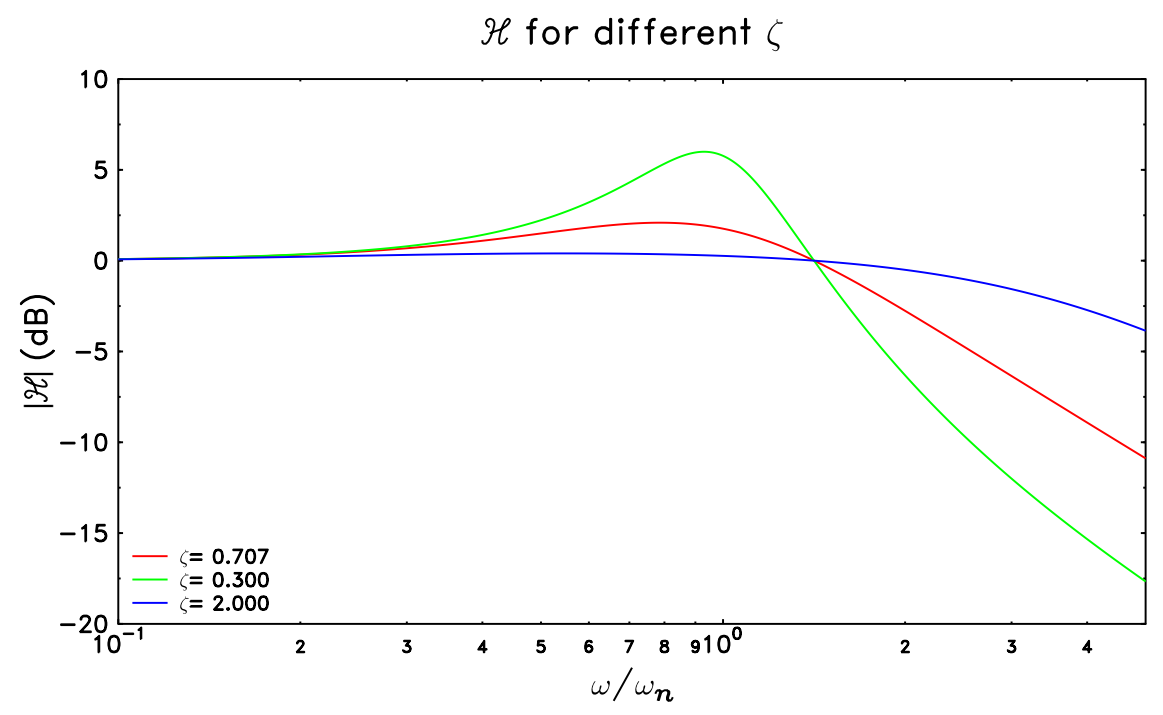

Figure 4 This is the Bode plot of $\mathcal{H}$ for different values of $\zeta$.

Again for completeness the normalized transfer function that relates $\theta_{0}$ to $\theta_{1}$ is

$$
\frac{\theta_{1}}{\theta_{0}}=\frac{K s^{3}+\frac{\omega_{n}^{2} s^{2}}{g K_{0} K_{d}}+2 s \zeta \omega_{n}+\omega_{n}^{2}}{s^{2}+2 s \zeta \omega_{n}+\omega_{n}^{2}} \quad \text { if } \quad \omega_{n} / g K_{0} K_{d} K \ll 2 \zeta
$$




\section{ERROR TRANSFER FUNCTION}

We will calculate the error transfer function $\mathcal{H}_{e}$ in this section. Our definition of the error $\theta_{e}$ in the phase lock is

$$
\theta_{e}=\theta_{0}-\theta_{2}
$$

This is a natural definition because we are interested in the error between the beam response centre frequency and our excitation.

So with this definition and using (4) for $\theta_{2}$ and (7) for $\theta_{1}=\mathcal{H} \theta_{0}$, we obtain

$$
\frac{\theta_{e}}{\theta_{0}}=\frac{s}{s+K_{0} K_{d} K G(s)} \equiv \mathcal{H}_{e}
$$

Clearly $s \rightarrow 0 \theta_{e} \rightarrow 0$ if $G(0) \neq 0$ which is true for low pass filters.

For the special case of the passive lag filter we can write $\mathcal{H}_{e}$ in normalized form

$$
\mathcal{H}_{e}(s)=\frac{s\left(s+\frac{\omega_{n}^{2}}{g K_{0} K_{d} K}\right)}{s^{2}+2 s \omega_{n} \zeta+\omega_{n}^{2}}
$$

The Bode plot of $\mathcal{H}_{e}(s)$ for different $\zeta$ values is shown in Figure 5.

For the case when $\omega_{n} / g K_{0} K_{d} K \ll 2 \zeta$, we can use the approximation $\mathcal{H}$ from (12) which gives

$$
\mathcal{H}_{e}(s)=\frac{s^{2}}{s^{2}+2 s \omega_{n} \zeta+\omega_{n}^{2}}
$$

Phase Step in Beam Response

If we have a phase step $\Delta \phi$ in $\Theta_{0}(t)$ then

$$
\theta_{0}(s)=\frac{\Delta \Phi}{s}
$$

Therefore the error $\theta_{e}$ from (15) is

$$
\theta_{e}=\frac{\Delta \Phi}{s+K_{0} K_{d} K G(s)}
$$




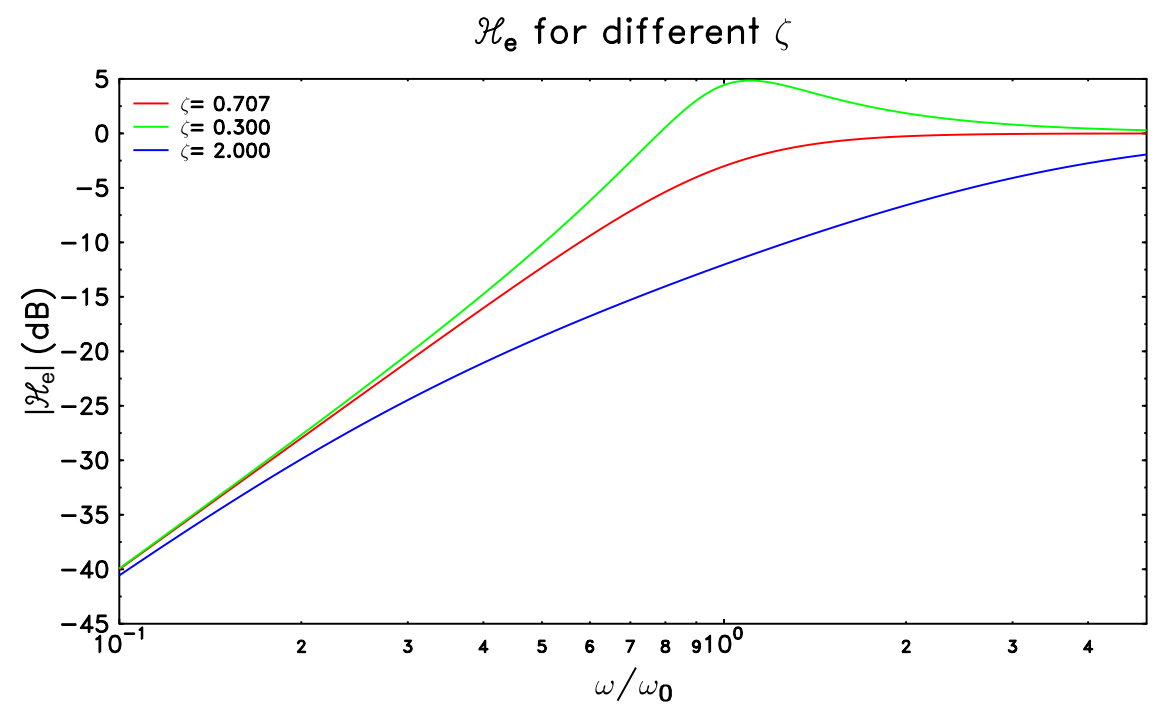

Figure 5 This is the Bode plot of $\mathcal{H}_{e}$ for different values of $\zeta$ for $K_{0} K_{d} K=10^{6}$ and $g=1$ time $^{-1}$.

Using the final value theorem

$$
\Theta_{e}(t=\infty)=\lim _{s \rightarrow 0} s \theta_{e}(s)=\lim _{s \rightarrow 0} \frac{s \Delta \Phi}{s+K_{0} K_{d} K G(s)}=0
$$

if $G(0) \neq 0$. This means that the PLL will converge to the right frequency when the beam response centre frequency has a phase step.

\section{Frequency Step in Beam Response}

If we have a frequency step $\Delta \omega$ in $\Theta_{0}(t)$ then

$$
\theta_{0}(s)=\frac{\Delta \omega}{s^{2}}
$$

Therefore the error $\theta_{e}$ from (15) is

$$
\theta_{e}=\frac{\Delta \omega}{s\left(s+K_{0} K_{d} K G(s)\right)}
$$


Again we can apply the final value theorem and find that

$$
\begin{aligned}
\Theta_{e}(t=\infty) & =\lim _{s \rightarrow 0} s \theta_{e}(s)=\lim _{s \rightarrow 0} \frac{\Delta \omega}{s+K_{0} K_{d} K G(s)}=\frac{\Delta \omega}{g K_{0} K_{d} K} \\
& =0 \quad \text { if } g K_{0} K_{d} K \gg \Delta \omega \text { and } G(0)=g
\end{aligned}
$$

This means that if there is a frequency step in the beam response centre frequency the PLL will converge to it eventually.

\section{Frequency Ramp in Beam Response}

If we have a frequency ramp $\Delta \dot{\omega}$ in $\Theta_{0}(t)$ then

$$
\theta_{0}(s)=\frac{\Delta \dot{\omega}}{s^{3}}
$$

For this part of the exercise we will use the special case of the passive lag filter. If $\omega_{n} / g K_{0} K_{d} K \ll 2 \zeta$ then we can use the approximation of $\mathcal{H}_{e}$ from (17)

$$
\mathcal{H}_{e}(s)=\frac{s^{2}}{s^{2}+2 s \omega_{n} \zeta+\omega_{n}^{2}}
$$

Therefore the error $\theta_{e}$ is

$$
\theta_{e}=\frac{\Delta \dot{\omega}}{s\left(s^{2}+2 s \omega_{n} \zeta+\omega_{n}^{2}\right)}
$$

and from the final value theorem

$$
\Theta_{e}(t=\infty)=\lim _{s \rightarrow 0} s \theta_{e}(s)=\lim _{s \rightarrow 0} \frac{\Delta \dot{\omega}}{s^{2}+2 s \omega_{n} \zeta+\omega_{n}^{2}}=\frac{\Delta \dot{\omega}}{\omega_{n}^{2}}
$$

Thus for a frequency ramp in the beam response there is an error that is not zero. If we demand that $\Theta_{e} \leq 1$ then the maximum rate of change of frequency that does not cause the PLL to lose lock is

$$
\Delta \dot{\omega}_{\max }=\omega_{n}^{2}
$$




\section{NO INTEGRATOR}

The integrator $1 / s$ in $F(s)$ of Figure 2 is important for the PLL to track in one

important condition: frequency ramp. The transfer function $\mathcal{H}^{\prime}$ which relates $\theta_{2}$ and $\theta_{0}$ without the integrator is

$$
\mathcal{H}^{\prime}(s)=\frac{K_{0} K_{d} K G(s)}{1+K_{0} K_{d} K G(s)}
$$

For the case of the passive lag filter, it is easy to show that

$$
\mathcal{H}^{\prime}(s)=\frac{\omega_{n}^{2}+2 s \zeta \omega_{n}}{s\left(1+2 \zeta \omega_{n}\right)+\omega_{n}^{2}} \quad \text { if } \omega_{n} / K_{0} K_{d} K g \ll 2 \zeta
$$

The error transfer function $\mathcal{H}_{e}^{\prime}$ is

$$
\mathcal{H}_{e}^{\prime}=\frac{s}{s\left(1+2 \zeta \omega_{n}\right)+\omega_{n}^{2}}
$$

Again, we will consider the three cases:

(i) Phase step $\Delta \Phi / s$. The tracking error converges to zero because as $t \rightarrow \infty$

$$
\Theta_{e}^{\prime}(t=\infty)=\lim _{s \rightarrow 0} \frac{s \Delta \Phi}{s\left(1+2 \zeta \omega_{n}\right)+\omega_{n}^{2}}=0
$$

(ii) Frequency step $\Delta \omega / s^{2}$. The tracking error converges to a finite value because as $t \rightarrow \infty$

$$
\Theta_{e}^{\prime}(t=\infty)=\lim _{s \rightarrow 0} \frac{\Delta \omega}{s\left(1+2 \zeta \omega_{n}\right)+\omega_{n}^{2}}=\frac{\Delta \omega}{\omega_{n}^{2}} \neq 0
$$

(iii) Finally for a frequency $\operatorname{ramp} \Delta \dot{\omega} / s^{3}$. The tracking error does not converge to a finite value because as $t \rightarrow \infty$

$$
\Theta_{e}^{\prime}(t=\infty)=\lim _{s \rightarrow 0} \frac{\Delta \dot{\omega}}{s\left[s\left(1+2 \zeta \omega_{n}\right)+\omega_{n}^{2}\right]} \rightarrow \infty
$$

This means that if the tune frequency is linearly ramped, the PLL will not lock. 


\section{PULSED EXCITATION}

The motivation behind using pulsed excitation rather than a continuous sine wave excitation comes from the observation of synchrotron lines in the tune spectra. See Fig-

ure 6. As we can see from these figures, the PLL can in principle lock onto any of these synchrotron lines.

In order to overcome this problem and always lock to the synchrotron line that is at the centre of the betatron tune, we have to somehow remove the effect of the synchrotron lines - if we are able to do this, we can then easily find the "true" tune. After some analysis, we realize that when we excite the beam continuously, the phase measurement of the beam w.r.t. the excitation is measured to infinite accuracy i.e. zero resolution bandwidth. In contrast, if we excite the beam for a finite amount of time, i.e. pulsed excitation, the resolution bandwidth becomes non-zero. And if we choose the pulse time such that its Fourier spectrum covers a few synchrotron lines, we can then smooth out the phase response around these synchrotron lines.

With this simple idea in mind, we see that when we turn the excitation on for $\tau \mathrm{s}$, the Fourier transform of a rectangular pulse with this width is

$$
\mathcal{F} \mathcal{T}\left[\operatorname{rect}\left(\frac{t}{\tau}\right)\right]=\tau \operatorname{sinc}\left(\frac{\omega \tau}{2}\right)
$$

with rect() the rectangle function defined as

$$
\operatorname{rect}(t)= \begin{cases}1 & \text { if }|t|<1 / 2 \\ 0 & \text { otherwise }\end{cases}
$$

and the $\operatorname{sinc}()$ function defined as

$$
\operatorname{sinc}(x)=\frac{\sin x}{x}
$$

The Fourier transform $\mathcal{F} \mathcal{T}[\omega]$ is the engineer's definition

$$
\mathcal{F} \mathcal{T}[\omega]=\int_{-\infty}^{\infty} d t e^{-i \omega t} f(t)
$$


Date: 05-03-01 Time: 07:08 PH

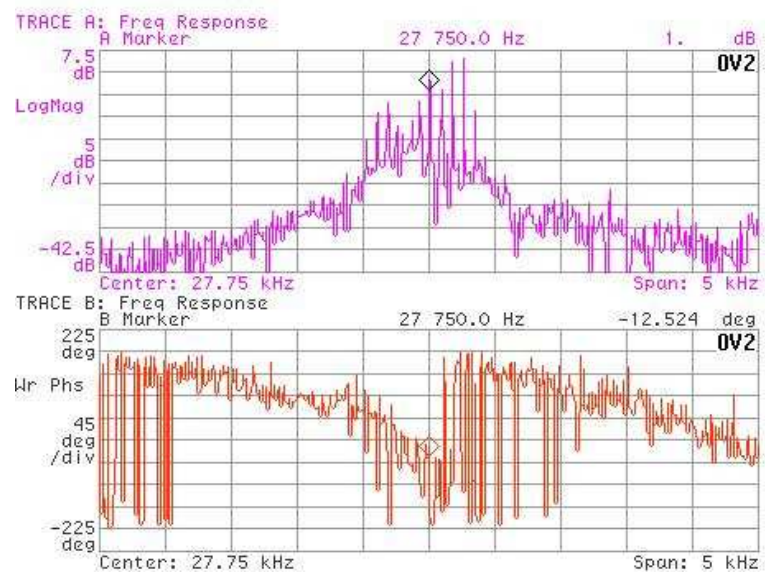

Date: 05-03-01 Time: 07:13 PM
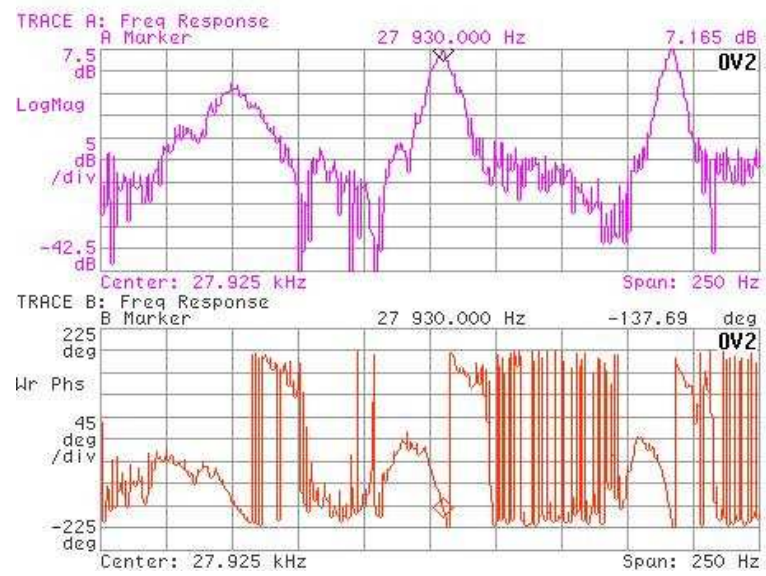

Figure 6 The horizontal frequency response of one coalesced bunch at $150 \mathrm{GeV}$ in the Tevatron. The chromaticity is about 8 units. Clearly seen are the synchrotron lines embedded in a background tune. The zoomed in view shows the problem: our PLL can arbitrarily lock to any one of them.

We can further define the bandwidth $\Delta_{\text {bw }}$ of the $\operatorname{sinc}()$ function in Fourier space to be the width at the zero crossing of its largest lobe shown in Figure 7. This gives us the formula

$$
\Delta_{\text {bw }}(\mathrm{Hz})=\frac{2}{\tau}
$$



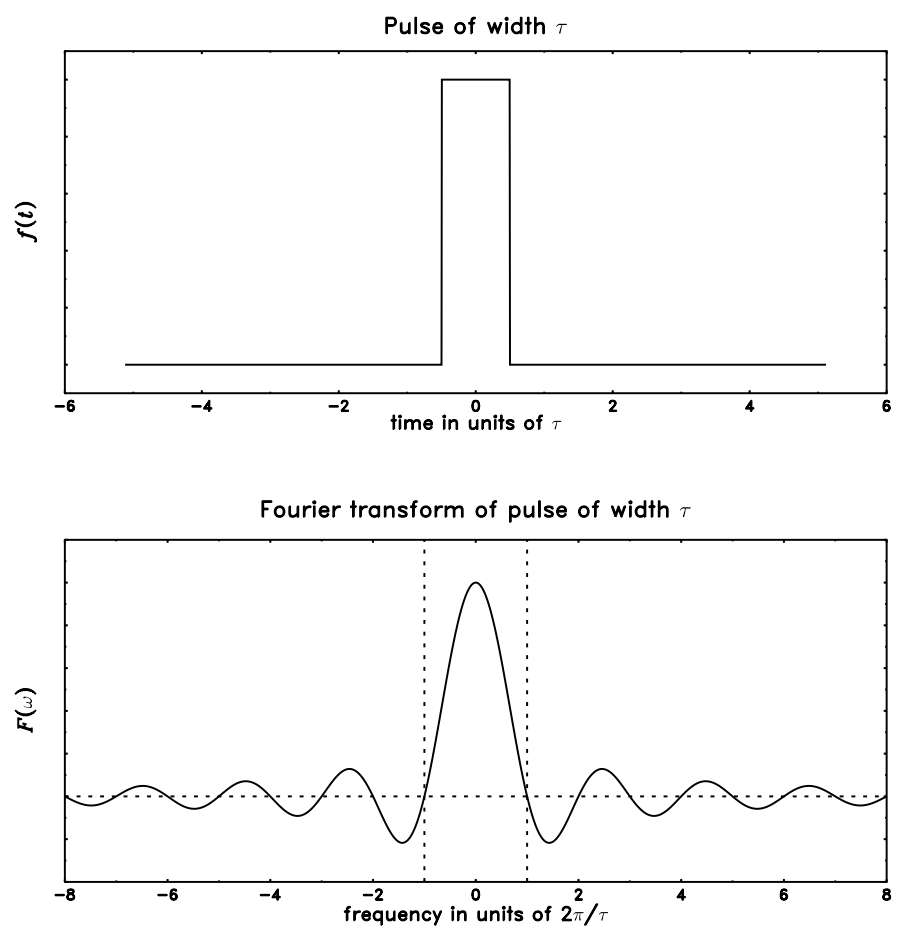

Figure 7 The Fourier transform of a pulse of width $\tau$ is a sinc function. The width of the largest lobe (enclosed by the vertical and horizontal dotted lines) in the bottom graph is $4 \pi / \tau \mathrm{rad} \cdot \mathrm{s}^{-1}$.

The value of $\tau$ is dictated by the synchrotron frequency. At $150 \mathrm{GeV}$, the synchrotron frequency is about $84 \mathrm{~Hz}$. If we choose $\tau=10 \mathrm{~ms}$, we have $\Delta_{\mathrm{bw}}=200 \mathrm{~Hz}$, this means that we will average a little over 2 synchrotron lines.

The amount of "off" time is dictated by the width of the synchrotron line, i.e. its $Q$ value. See Figure 6 . The half-width at half-max $f_{\frac{1}{2}, \frac{1}{2}}$ of a synchrotron line is about $\sim 5 \mathrm{~Hz}$. This means that the $1 / e$ time is about $1 / 2 \pi f_{\frac{1}{2}, \frac{1}{2}} \approx 30 \mathrm{~ms}$ if we assume that the resonance has the shape of a Lorentzian. From empirical observations, a 1/e "off" time is sufficient to smooth out the synchrotron lines to reveal the background tune. See Figure 8. 


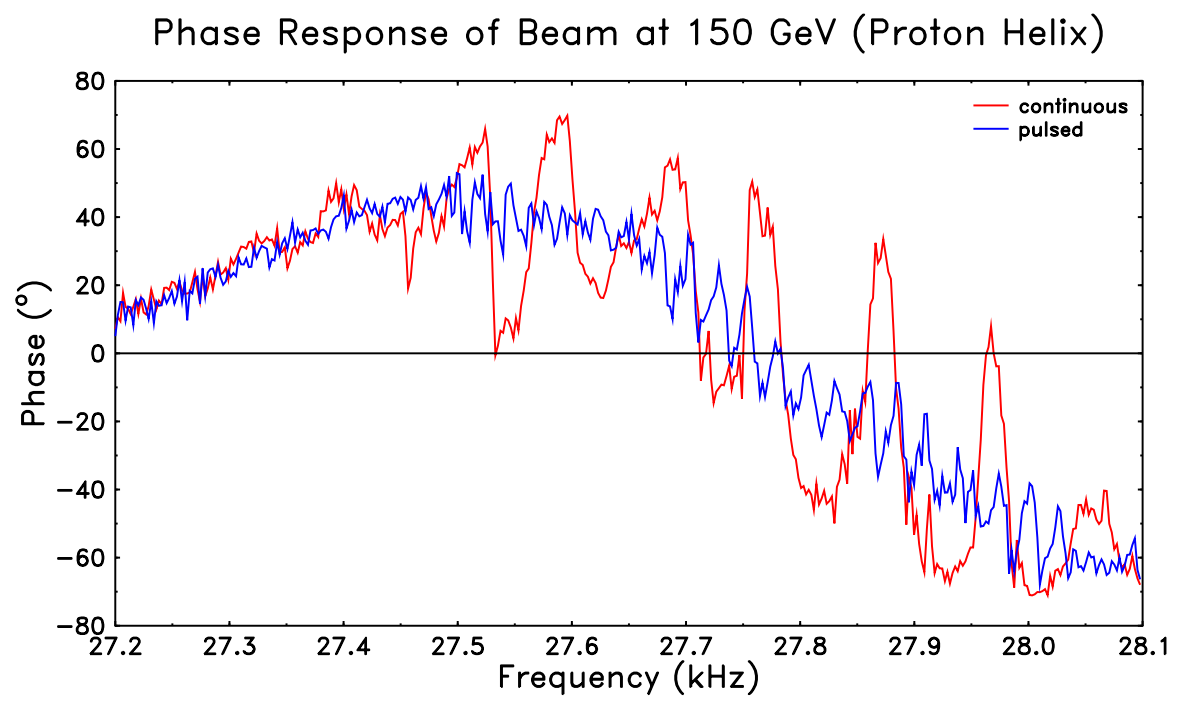

Figure 8 The horizontal phase response for a bunch of coalesced protons on the proton helix at $150 \mathrm{GeV}$ with continuous and pulsed excitation. The chromaticity is about 4 units. The pulsed excitation (10 ms on, $30 \mathrm{~ms}$ off) clearly smoothed out the phase response.

Another Possible Advantage

Another possible advantage of the pulsed excitation over continuous excitation is when we have both planes of the PLL commissioned. There is a possibility that coupling between the two planes may cause either of the PLLs to lose lock because continuous excitation in one plane will cause the other plane to be excited as well. The solution may be as simple as staggering the pulsing between the two planes, i.e. the horizontal pulsed PLL is on while the vertical PLL is off and vice versa may be able to alleviate this potential problem. 


\section{Implementation}




\section{IMPLEMENTATION OVERVIEW}

In this section, we will discuss the implementation overview of the PLL tune tracker.

See Figure 9. The entire PLL tune tracker system can be broken down into the following major parts

( $i$ ) DSP motherboard and daughtercard. The heart of the PLL.

(ii) The software PLLs which live in the DSP. The brains of the PLL.

(iii) The kicker feedback circuit. ${ }^{\dagger}$ This circuit keeps the kicker power at a level that is high enough so that the PLL will still lock to the beam without blowing up the emittance.

(iv) Schottky detector. A resonant detection circuit which is extremely sensitive to the Schottky noise of the beam.

$(v)$ The automatic gain control circuit. This circuit keeps the input the analogue to digital converter (ADC) at a fixed level independent of beam conditions.

(vi) The phase compensator circuit. $\ddagger$ When the Tevatron ramps, the RF frequency increases from 53.103 MHz to $53.104 \mathrm{MHz}$. This increase leads to an additional phase shift in the measured phase of the beam response. The phase compensator circuit is designed to remove this extra phase.

In the following subsections, we will discuss each part separately.

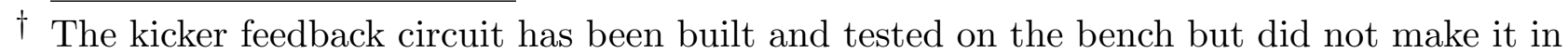
time before the August 2004 shutdown.

$\ddagger$ The phase compensator was not built for these experiments. 
Figure 9 The tune tracker PLL implementation block diagram. 


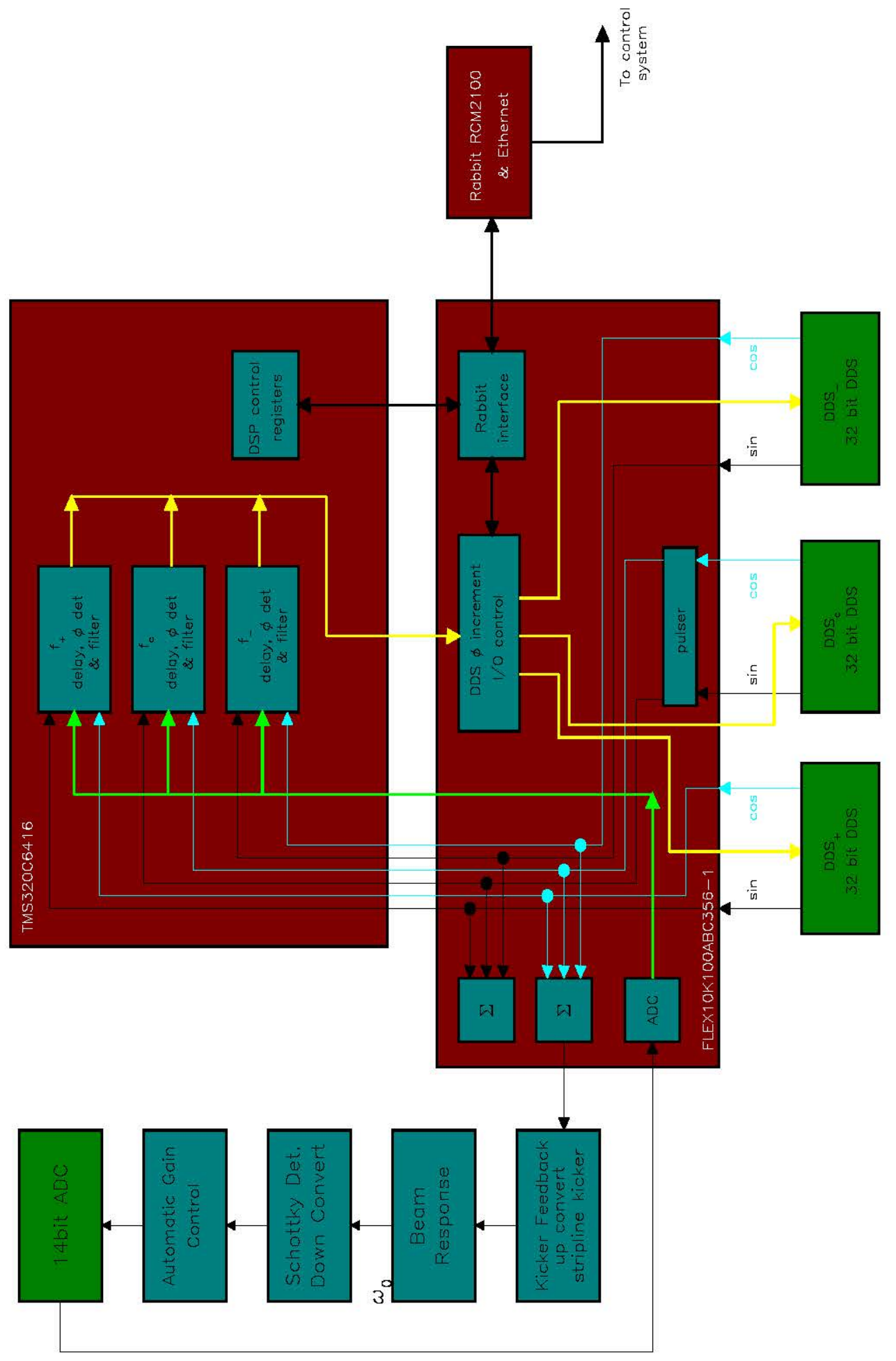




\section{DSP MOTHERBOARD AND DAUGHTERCARD}

The DSP motherboard and daughtercard is shown in Figure 10. The software PLL executes on the TMS320C6416 32-bit fixed-point digital signal processor (DSP) running at $500 \mathrm{MHz}$. We did not design the DSP motherboard but instead bought an off-the-shelf test and evaluation board TMS320C6416TEB from Texas Instruments. This motherboard has slots for plugging in daughtercards like analogue to digital converters (ADCs), digital to analogue converters (DACs) etc. which we used for our PLL tune tracker daughtercard.

The daughtercard for the PLL tune tracker is an inhouse design which at its heart is a 356-pin field programmable gate array (FPGA) made by ALTERA (EPF10K100ABC3561). All the input/output lines from every digital part on the daughtercard goes to or originates from the FPGA. The other integrated circuits on the daughtercard are:

(i) One 14-bit ADC from Texas Instruments (THS14F04) sampling at $300 \mathrm{Khz}$. This is used to digitize the Schottky signal. Note that in the DSP, we are looping over three software PLLs, so effectively, each software PLL is sampling at $100 \mathrm{kHz}$.

(ii) Three 32-bit direct digital synthesizers (DDSs) made by Qualcomm (Q2368) running at $25 \mathrm{MHz}$. Each DDS can produce two separate channels of digital sine and cosine waves of the same frequency at the same time. We designed the daughterboard to contain three DDSs because we want to be able to do multitone PLL, i.e. tune tracking with multiple excitations in order to lock to different synchrotron lines. The Q2368 was selected because unlike newer DDSs it does not contain an onchip DAC. This means that the DDS outputs can be routed back to the FPGA to do hardware addition without an intermediate ADC.

(iii) Two 14-bit DACs from Analog Devices (AD9764). One of the DACs converts the digitally summed sine signal of the three DDSs to analogue while the other does 
the same for the summed cosine digital signal.

Hardware control of the ADC and the DDSs by the DSP comes from writing to and reading from the control registers on the FPGA via the above mentioned slot on the TEB.

The control system controls the DSP over the ethernet by communicating with a microcontroller daughtercard (RCM2100) that has onboard ethernet. The RCM2100 talks to the DSP by writing to and reading from registers on the FPGA. 
Figure 10 The daughter card which plugs directly into an off the shelf DSP card (TMS320C6416TEB) is shown here. 


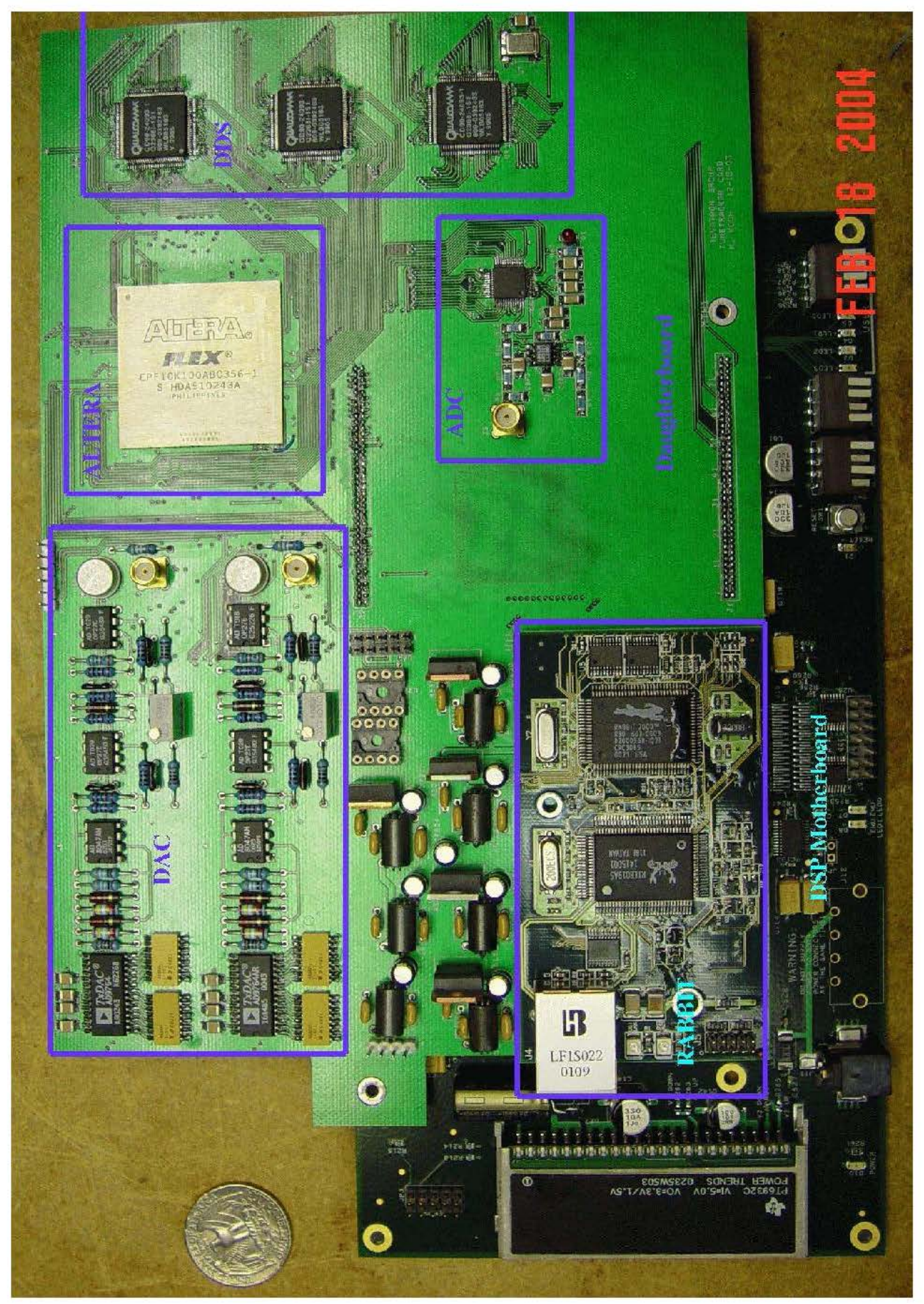




\section{DSP SOFTWARE PLL}

The software running on the DSP is the brains of the operation. The block diagram of the software which is running in the DSP is shown in Figure 11. There are three software PLLs running on the DSP. Two of them are continuous excitation PLLs and one of them is a pulsed excitation PLL. Figure 11 shows that the continuous and pulsed PLLs are nearly identical in structure to the PLL described by Figure 2 in the Theory section. We see that for the continuous excitation PLL the only addition is the gain stage $A$. For the pulsed excitation PLL, the filter and integrator are updated only during the time when the excitation is on. The on/off time of the pulsed excitation has already been discussed in section Pulsed Excitation and will not be touched here. For completeness, the implementation values and units of $g, K_{0}, K_{d}$ and $K$ are summarised in Table 1.

\begin{tabular}{c|c|c|l}
\hline \multicolumn{3}{c}{ Table 1. Implementation Values of Software PLL } \\
\hline Variable & Value & Units & \multicolumn{1}{c}{ Remarks } \\
$g$ & $10^{5}$ & $\mathrm{~s}^{-1}$ & integrator gain \\
$K_{0}$ & 0.0366 & $\mathrm{rad} \cdot \mathrm{s}^{-1} / \mathrm{c} . \mathrm{u}$. & DDS gain \\
$K_{d}$ & 0.25 & $\mathrm{c.u} \cdot / \mathrm{rad}$ & phase detector gain \\
$K$ & $(0.2$ to 2$) \times 10^{-2}$ & $\mathrm{~s}$ & slope of beam phase response \\
$A$ & 1 to 20 & - & programmable gain multiplier \\
\hline \hline
\end{tabular}

The values in Table 1 are calculated like this:

(i) $g$ the integrator gain comes from normalizing the sampling time $d t$ so that $g \times d t=$ 1. For a sampling frequency of $100 \mathrm{kHz}, d t=10^{-5} \mathrm{~s}$, thus $g=10^{5} \mathrm{~s}^{-1}$.

(ii) $K_{0}$ the DDS gain comes from converting a 32-bit number into frequency. For a DDS clock frequency of $25 \mathrm{MHz}$, every computer unit (c.u.) in decimal corresponds to $2 \pi \times\left(25 \times 10^{6}\right) / 2^{32}=0.0366 \mathrm{rad} \cdot \mathrm{s}^{-1} / \mathrm{c} . \mathrm{u}$. 
Continuous Excitation PLL

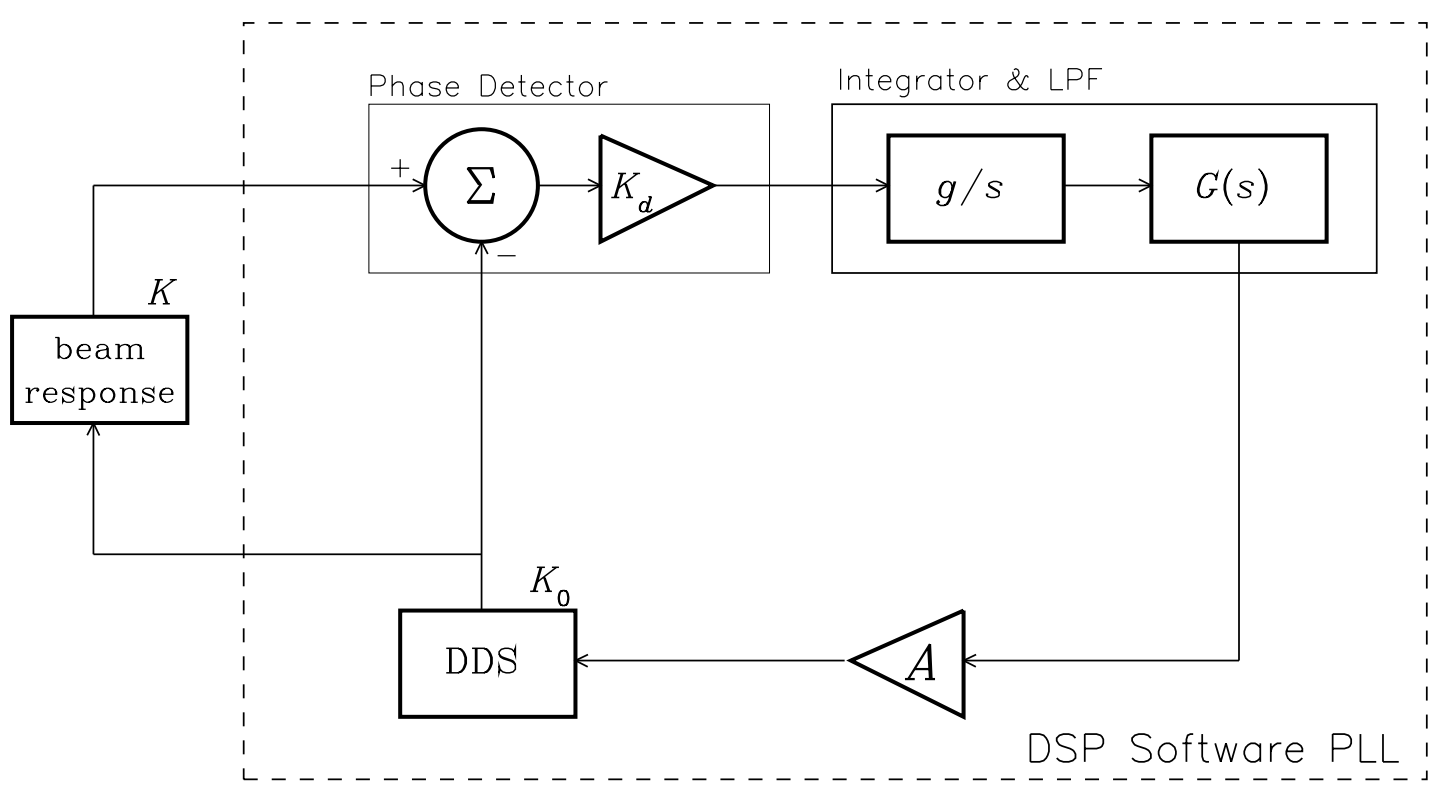

Pulsed Excitation PLL

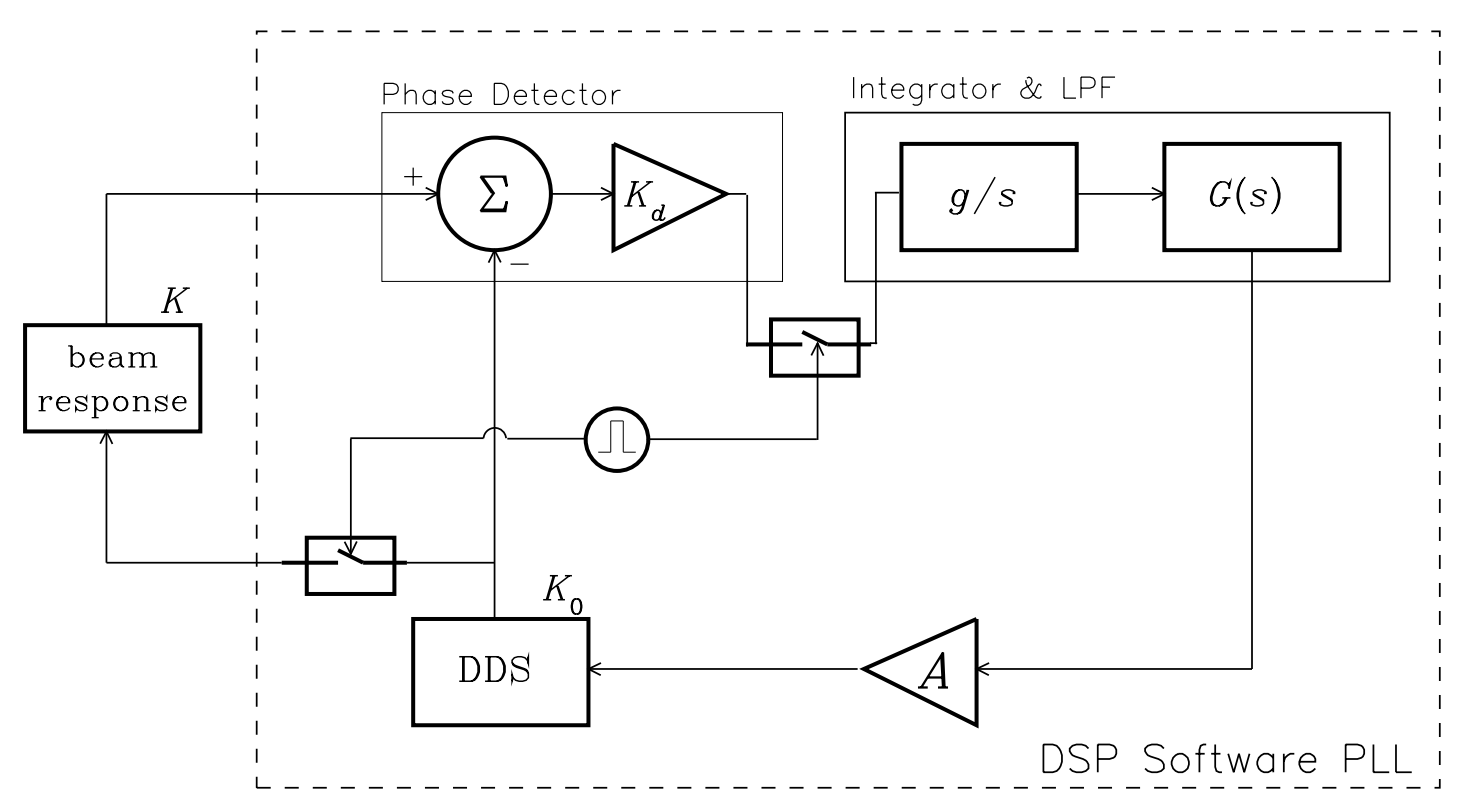

Figure 11 The block diagram of the software PLLs implemented in the DSP. There are three software PLLs, two continuous excitation and one pulsed excitation. 
(iii) $K_{d}$ is the phase detector gain. Our phase detector which will be described in the section Phase Detector returns phase in the decimal range $\pm \pi$. However, since our DSP is a fixed point processor, we need to keep the phase confined to the decimal range \pm 1 . Thus, we divide the phase by 4 to keep it in this range.

(iv) $K$ is the slope of the beam phase response. The phase slope is measured from the phase response measurement shown in Figure 8. The measured smoothed slope from pulsed excitation is about $0.2 \times 10^{-2} \mathrm{~s}$ and from continuous excitation, the slope from one synchrotron line is about $2 \times 10^{-2} \mathrm{~s}$.

With these values out of the way, the most important part to discuss next is the phase detector. Without a good and robust phase detector the entire system will not work. Right after the phase detector is the low pass filter $G(s)$. We will implement $G(s)$ as an infinite impulse response (IIR) filter. The coefficients of this filter are determined by the phase response of the beam. The calculation of these coefficients will be shown after the phase detector discussion.

\section{Phase Detector}

The purpose of the phase detector is to detect the phase difference between the input sine wave and a reference sine wave. Our implementation of the phase detector is shown in Figure 12.

Before going into the mathematics of the block diagram, we will quickly discuss the pur-

pose of the Hilbert transformer (which will be discussed in detail in the next subsection). ${ }^{4}$ The Hilbert transformer is a filter that is able to exactly phase shift an input sine wave by $\pi / 2$, i.e.

$$
\sin \left(\omega t^{\prime}+\theta\right) \rightarrow \text { Hilbert Transformer } \rightarrow \cos \left(\omega t^{\prime}+\theta\right)
$$

Of course, nothing is for free: an $(N+1)$ tap Hilbert Transformer has a delay of $N / 2$ 


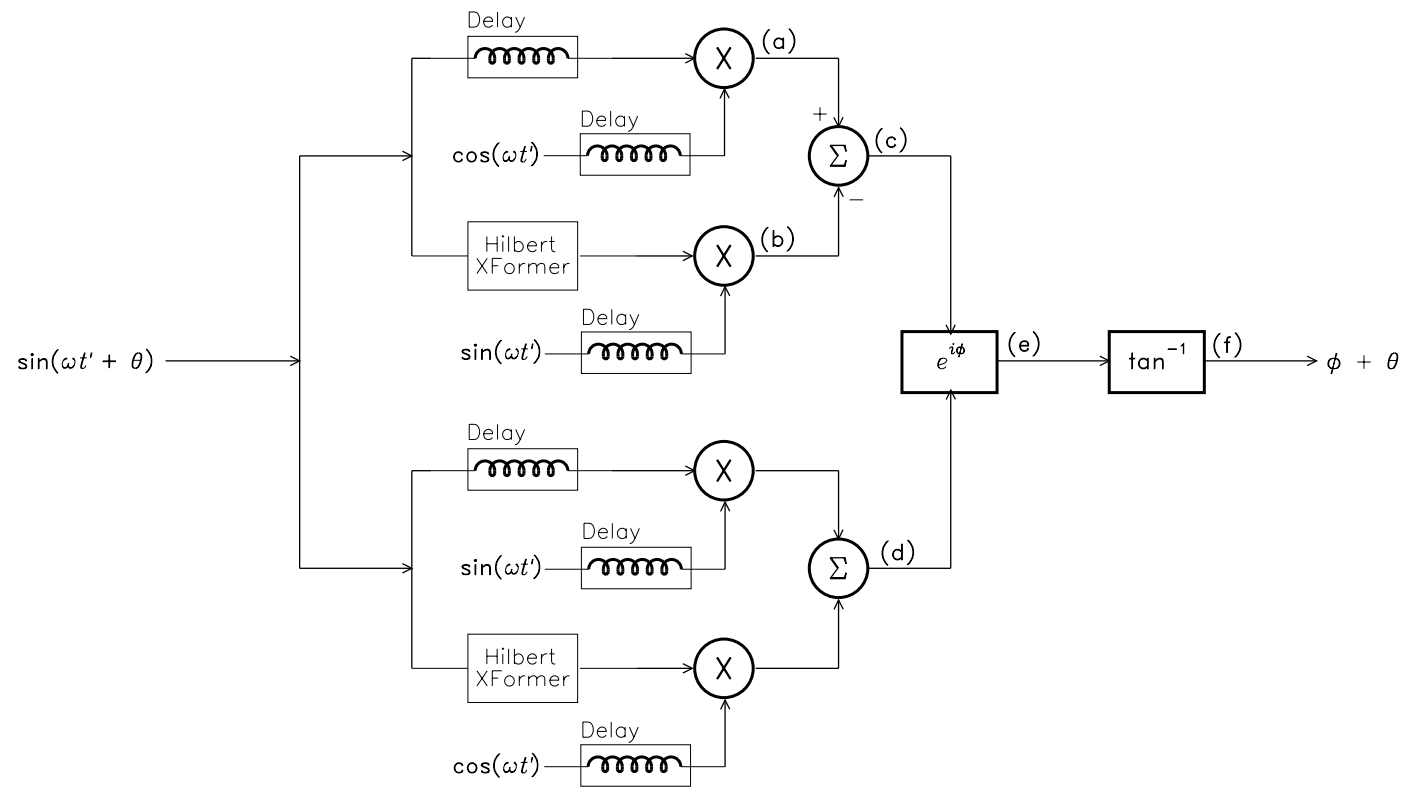

Figure 12 The phase detector. Note that $\phi$ is a user input phase offset, i.e. $\phi$ can be set to zero by the user if a phase offset is not needed.

samples. (This means that for a 51 tap transformer, there is a delay of 25 samples or $250 \mu \mathrm{s}$ at $100 \mathrm{kHz}$ sampling rate). Thus, this requires us to add a delay of exactly $\mathrm{N} / 2$ samples at various points shown in the block diagram.

So we start with the phase detector analysis by looking at point $(a)$ in Figure 12. At time $t^{\prime}$ we have

$$
(a)=\sin (\omega t+\theta) \cos \omega t
$$

where $t$ is earlier than $t^{\prime}$ by $N / 2$ samples. At point (b), we have

$$
(b)=\cos (\omega t+\theta) \sin \omega t
$$

Taking the difference between $(a)$ and $(b)$, we get at $(c)$

$$
\left.\begin{array}{rl}
(c) & =\sin (\omega t+\theta) \cos \omega t-\cos (\omega t+\theta) \sin \omega t \\
& =\sin \theta
\end{array}\right\}
$$


Similarly at $(d)$, we have

$$
\left.\begin{array}{rl}
(d) & =\sin (\omega t+\theta) \sin \omega t+\cos (\omega t+\theta) \cos \omega t \\
& =\cos \theta
\end{array}\right\}
$$

At point $(e)$ we introduce a phase rotation $\phi$ which will allow us take out any phase offsets. See Figure 13. It is obvious that we can think of $\sin \theta$ and $\cos \theta$ as projections of a unit vector $v$ onto the $x$ and $y$ axis of a Cartesian plane. In this plane, $\boldsymbol{v}$ when rotated by $\phi$ is given by the matrix transformation

$$
\left[\begin{array}{cc}
\cos \phi & -\sin \phi \\
\sin \phi & \cos \phi
\end{array}\right]\left[\begin{array}{c}
\cos \theta \\
\sin \theta
\end{array}\right]=\left[\begin{array}{c}
\cos (\theta+\phi) \\
\sin (\theta+\phi)
\end{array}\right] \equiv \boldsymbol{v}^{\prime}
$$

Therefore the angle $\boldsymbol{v}^{\prime}$ makes with the $x$-axis is simply

$$
\tan ^{-1}\left[\frac{\sin (\theta+\phi)}{\cos (\theta+\phi)}\right]=\theta+\phi \quad \text { where }-\pi \leq(\theta+\phi)<\pi
$$

The rotation method naturally limits the result of adding the phase offset $\phi$ to $\theta$ to $\pm \pi$.
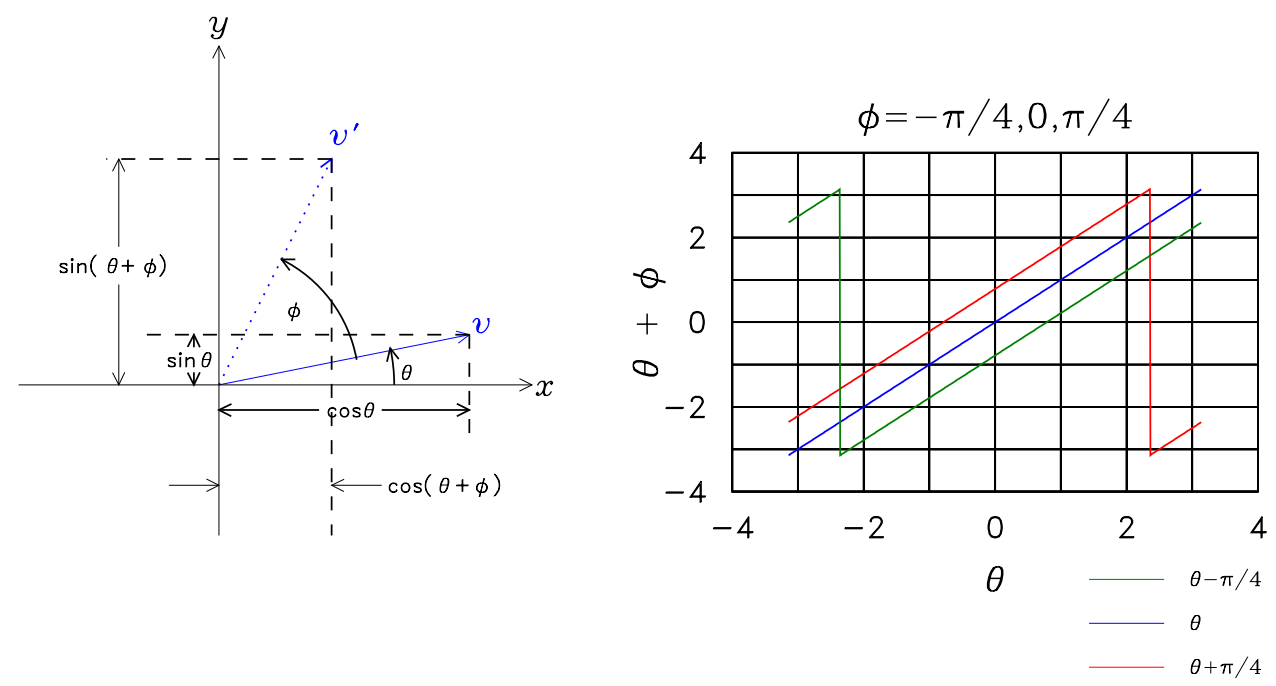

Figure 13 This shows that by rotating the unit vector $\boldsymbol{v}$ by $\phi$ to $\boldsymbol{v}^{\prime}$, we can introduce a phase offset. When we perform the $\tan ^{-1}$ after the rotation, the result is always confined to $\pm \pi$. 


\section{Hilbert Transformer}

The key to the phase detector is the use of a Hilbert Transformer for phase shifting the input sine wave by $\pi / 2$. Our Hilbert Transformer contains 51 taps whose coefficients are shown in Table 4 of Appendix II. The magnitude and phase response is shown in Figure 14. Clearly the Hilbert transformer phase shifts the input by $\pi / 2$ with possibly a small change in magnitude $\pm 0.15 \mathrm{~dB}$, as long as the input frequency is between $0.05 / T_{s}$ to $0.45 / T_{s}$ where $T_{s}$ is the sampling period. For example, when the sampling frequency is $100 \mathrm{kHz}$, the Tevatron tune frequency $\sim 27.5 \mathrm{kHz} \approx 0.55 \times \pi$ lies right in the middle of the Hilbert transformer phase response.
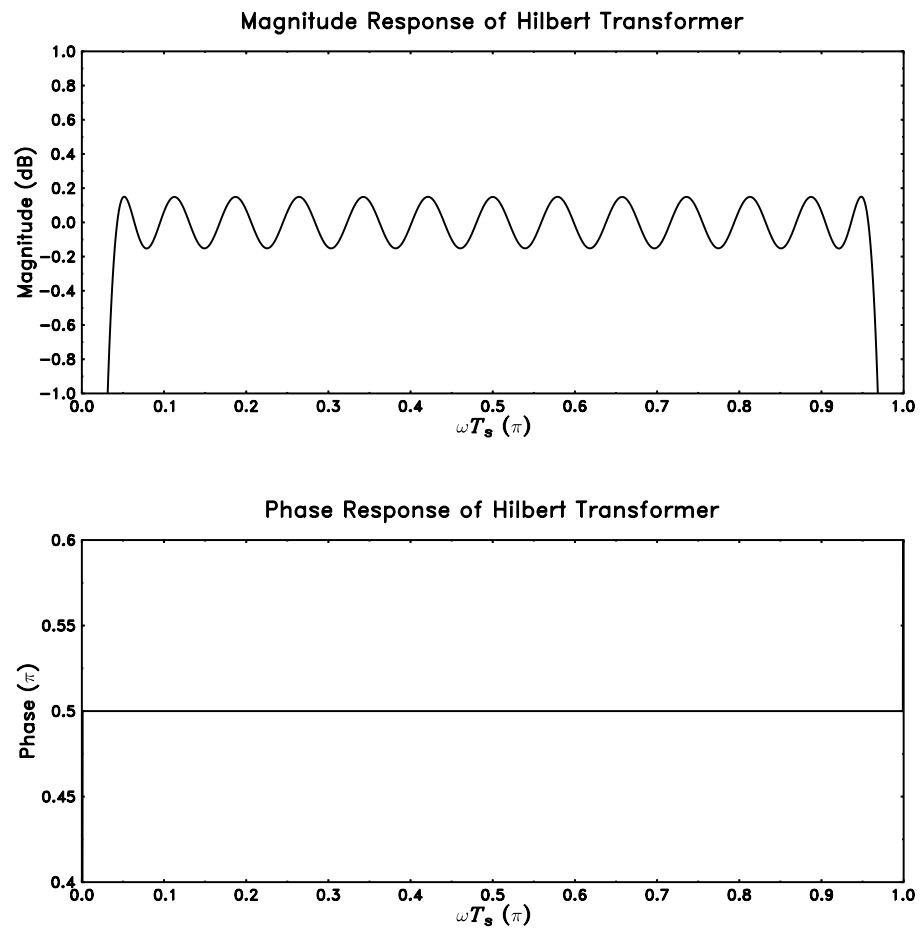

Figure 14 The frequency response of the Hilbert transformer using the coefficients shown in Table 4 . 


\section{PLL Parameters from Beam Phase Response}

The beam phase response will determine the natural frequency $\omega_{n}$, and the form of the low pass filter $G(s)$, These values will then determine the other loop parameters like the loop bandwidth, lock-in time, pull in range etc.

For calculating the natural frequency $\omega_{n}$, we notice that from (7), the PLL transfer function $\mathcal{H}(s)$ is a $2^{\text {nd }}$ order linear phase lock loop if it does not wander too far away from the resonance. If this condition is satisfied we can use the results derived in the Theory section and compare them to the transfer function derived in Best ${ }^{5}$ section 2.3 . We will find that they are the same except for extra factors $g, K$ and $A$ shown in Table 1. This means that we can lift some of the formulæ and arguments used by Best and apply them here.

First, we want the PLL to lock within one single beat if the difference between the beam resonant frequency and the excitation frequency is less than $\Delta \omega_{L}$, called the lock range. We want $\Delta \omega_{L}$ to be at least the width of one synchrotron line which is about $5 \mathrm{~Hz}$ (measured from Figure 6) since the linear theory only works when it is close to resonance i.e.

$$
\Delta \omega_{L} \sim 2 \pi \times 5 \mathrm{rad} \cdot \mathrm{s}^{-1}
$$

The lock range is related to the natural frequency by $\Delta \omega_{L} \approx 2 \zeta \omega_{n}$ if $\omega_{n} / g K_{0} K_{d} K A \ll 2 \zeta .^{\dagger}$ If we want an optimally flat PLL transfer function then $\zeta=0.7$, thus

$$
\left.\begin{array}{rl}
\Delta \omega_{L} & \approx 2 \zeta \omega_{n}=1.4 \times \omega_{n} \\
\therefore \quad \omega_{n} & =2 \pi \times 5 / 1.4=(2 \pi \times 3.5) \mathrm{rad} \cdot \mathrm{s}^{-1}
\end{array}\right\}
$$

\section{Calculating $G(s)$}

In principle, we can calculate the form of $G(s)$ with $\omega_{n}=2 \pi \times 3.5 \mathrm{rad} \cdot \mathrm{s}^{-1}$ found in

$\dagger$ We will see that this condition is not satisfied in Calculating $G(s)$, but we will nevertheless use this as a starting point for $\omega_{n}$. 
(47) and with the choice of $\zeta=0.7$ for an optimally flat PLL transfer function provided that we know $g, K_{0}, K_{d}, K$ and $A$. If we solve for $\tau_{1}$ and $\tau_{2}$ from (11) after we set these values from Table 1 and take $K=0.6 \times 10^{-2} \mathrm{~s}$ (which is the phase slope of the "fake beam response" circuit. See section Closed Loop Response of the Continuous PLL) and $A=3$, we find that $g K_{0} K_{d} K A \approx 16.5 \mathrm{~s}^{-1}$. Fortunately, (11) does not need to satisfy the condition $\omega_{n} / g K_{0} K_{d} K A \ll 2 \zeta$ and thus we can find $\tau_{1}+\tau_{2}$ and $\tau_{2}$

$$
\left.\begin{array}{rl}
\tau_{1}+\tau_{2} & =\frac{g K_{0} K_{d} K A}{\omega_{n}^{2}}=16.5 /(2 \pi \times 3.5)^{2}=0.03 \mathrm{~s} \\
\tau_{2} & =\frac{2 \zeta}{\omega_{n}}-\frac{1}{g K_{0} K_{d} K A} \approx \frac{2 \times 0.7}{2 \pi \times 3.5}-\frac{1}{16.5}=0.003 \mathrm{~s}
\end{array}\right\}
$$

Unfortunately, this filter is rather poor at suppressing high frequency noise because $\tau_{2}$ is only 10 times smaller than $\left(\tau_{1}+\tau_{2}\right)$. See Figure 16 . We can improve it by decreasing $\tau_{2}$ by a factor of 10 and still get approximately the same $\zeta$, i.e. if $\tau_{2}=0.3 \times 10^{-3}$, we have

$$
\left.\begin{array}{rl}
\zeta & =\frac{\omega_{n}}{2}\left(\tau_{2}+\frac{1}{g K_{0} K_{d} K A}\right)=\frac{2 \pi \times 3.5}{2} \times\left(0.3 \times 10^{-3}+\frac{1}{16.5}\right) \\
& =0.67 \approx 0.7
\end{array}\right\}
$$

Thus, with this new value of $\tau_{2}$ the edges of the passive lag filter shown in Figure 3 are

$$
\left.\begin{array}{l}
\omega_{b}=\frac{1}{\tau_{1}+\tau_{2}}=\frac{1}{0.03}=33 \mathrm{~s}^{-1} \\
\omega_{a}=\frac{1}{\tau_{2}}=\frac{1}{0.3 \times 10^{-3}}=3 \times 10^{3} \mathrm{~s}^{-1}
\end{array}\right\}
$$

For the digital realization of this filter, we implement it as an infinite impulse response (IIR) filter shown in Figure 15. The coefficients of the IIR come from

$$
\left.\begin{array}{rl}
y_{n} & =\frac{b}{T_{s}+b} y_{n-1}+\frac{T_{s}+a}{T_{s}+b} x_{n}-\frac{a}{T_{s}+b} x_{n-1} \\
& \equiv \alpha_{-1} y_{n-1}+\beta_{0} x_{n}-\beta_{-1} x_{n-1} \\
& \approx y_{n-1}+0.0113 x_{n}-0.0110 x_{n-1}
\end{array}\right\}
$$

where $a=1 / \omega_{a}$ and $b=1 / \omega_{b}$ and $T_{s}=10 \mu \mathrm{s}$ is the sampling time. Figure 15 and Figure 16 show the realization of $G(s)$ in frequency space. 


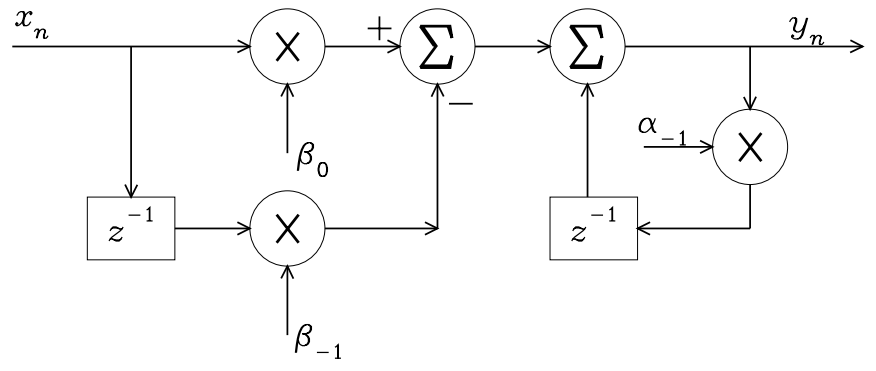

Figure 15 The equivalent IIR circuit of a passive lag filter drawn in the usual $z$-transform way. $z^{-1}$ represents a one sample delay.
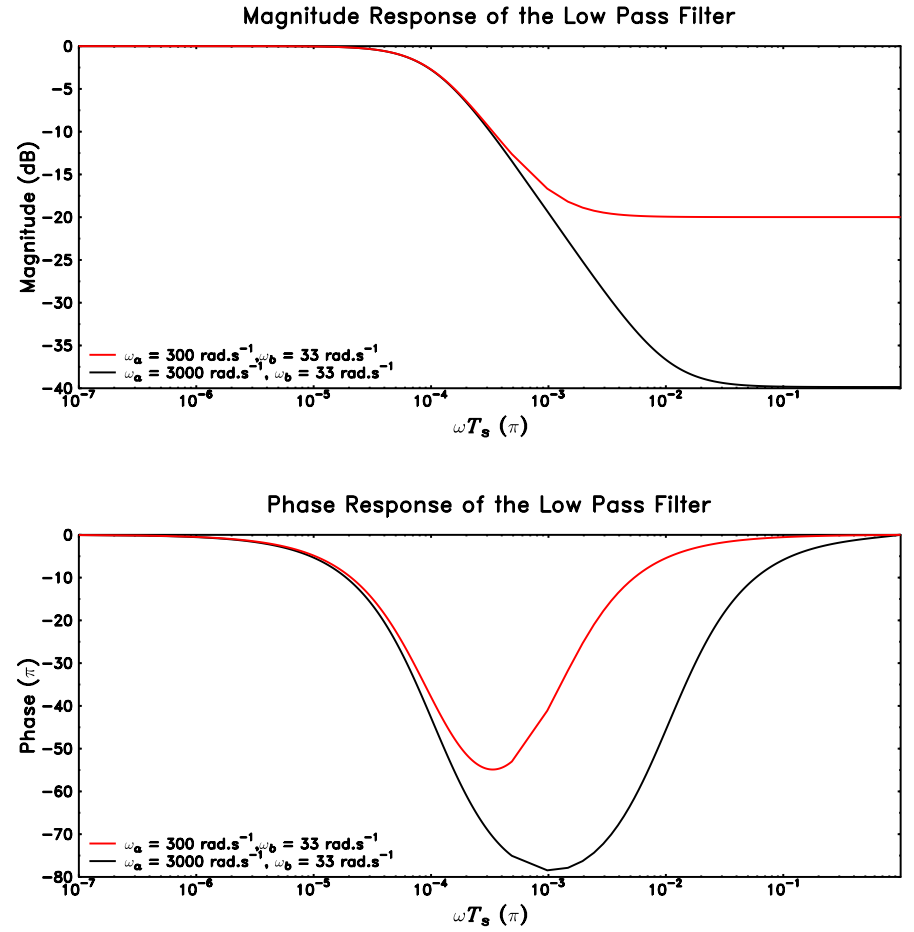

Figure 16 The frequency response of the low pass filter with the coefficients given by (48) (red) and (51) (black). 


\section{KICKER FEEDBACK}

The goal of the kicker feedback circuit is to keep the $S / N$ ratio to $\geq 6 \mathrm{~dB}$ which we will discuss in Minimum Kicker Power. The kicker power will be adjusted by the feedback loop so that the $S / N$ ratio is maintained. In our design the kicker feedback circuit consists of two major parts:

(i) Fourier component detector. The Fourier component detector picks out the Fourier component at the kicker frequency.

(ii) $S / N$ ratio detector. The $S / N$ ratio detector measures the ratio between the power from the kicker and the total power (less the kicker power) in the bandwidth which contains the tune. In this case, our bandpass filter is centred around $27.5 \mathrm{kHz}$ with a bandwidth of $5 \mathrm{kHz}$.

\section{Fourier Component Detector}

To extract out the PLL excitation Fourier component from the Schottky signal (see Figure 17), we start at the input from the AGC (which is from the Schottky detector). We must AC couple this into the multipliers because we do not want any DC contributions after multiplication. We assume that this input $y_{\text {in }}\left(\omega^{\prime}\right)$ has the form $y_{\text {in }}\left(\omega^{\prime}\right)=A \sin \left(\omega^{\prime} t+\phi\right)$. Let us consider first the case when $\omega^{\prime}=\omega$ the PLL excitation frequency, then at $(a)$

$$
\left.\begin{array}{rl}
(a) & =A \sin (\omega t) \sin (\omega t+\phi) \\
& =-\frac{A}{2}[\cos (2 \omega t+\phi)-\cos \phi]
\end{array}\right\}
$$

The $100 \mathrm{~Hz}$ low pass filter will leave us with the DC component of (52), i.e. $\frac{A}{2} \cos \phi$, if $(2 \omega>2 \pi \times 100) \mathrm{s}^{-1}$. After we square this, we obtain at $(c)$

$$
(c)=\frac{A^{2}}{4} \cos ^{2} \phi
$$




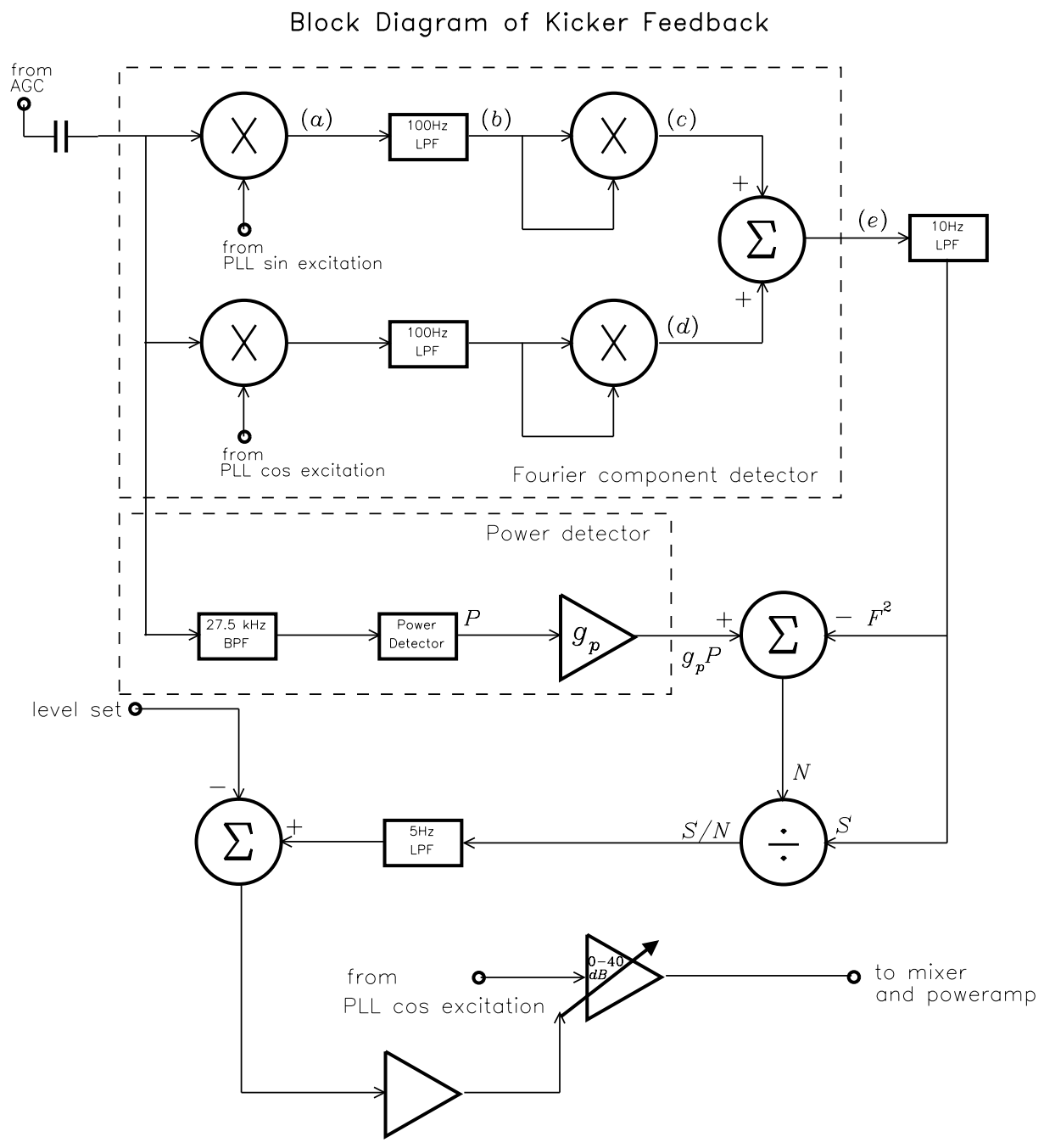

Figure 17 The block diagram of the kicker feedback circuit.

A similar argument shows that at $(d)$, we have

$$
(d)=\frac{A^{2}}{4} \sin ^{2} \phi
$$

Going through the summer,

$$
(c)+(d)=(e)=\frac{A^{2}}{4} \equiv F^{2}
$$

which is the Fourier component of the PLL excitation from the Schottky signal. 
For the case when $\omega^{\prime} \neq \omega$, we have at $(a)$,

$$
\left.\begin{array}{rl}
(a) & =A \sin (\omega t) \sin \left(\omega^{\prime} t+\phi\right) \\
& =-\frac{A}{2}\left\{\cos \left(\left[\omega^{\prime}+\omega\right] t+\phi\right)-\cos \left(\left[\omega-\omega^{\prime}\right] t-\phi\right)\right\}
\end{array}\right\}
$$

If $\left(\omega^{\prime}-\omega\right)>(2 \pi \times 100) \mathrm{s}^{-1}$, the low pass filter will suppress this. This means that the Fourier component detector will have contributions from $\pm 100 \mathrm{~Hz}$ around the PLL kicker frequency.

\section{Power Detector}

The power detector shown in Figure 17 integrates the power in the frequency band centred at $27.5 \mathrm{kHz}$ and a bandwidth of $5 \mathrm{kHz}$. The power detector is based on the Analog Devices AD8361 ${ }^{6}$ which measures the root mean square voltage from the input signal. To obtain power from this, we simply square the output of the AD8361.

\section{Normalized Signal to Noise Ratio $\overline{S / N}$}

We define the normalized signal to noise ratio $\overline{S / N}$ to be

$$
\overline{S / N}=\frac{F^{2}}{g_{p} P-F^{2}}
$$

where $F^{2}$ is the output of the Fourier detector and $P$ the output from the power detector and $g_{p}$ is the gain at the output of the power detector. Its value is adjusted so that when there is no noise at the AGC input, $g_{p} P \equiv 2 \times F^{2}$. Therefore,

$$
(\overline{S / N})_{\text {no noise }}=\frac{F^{2}}{2 \times F^{2}-F^{2}}=1
$$

The reasons for our $\overline{S / N}$ definition are as follows:

(i) We want to remove any contribution of $F^{2}$ from noise because (a) it is a coherent signal and (b) in most cases when the PLL is locked $F^{2}$ is larger than the 
background noise by at least $6 \mathrm{~dB}$ and thus it can overwhelm any background noise.

(ii) The usual definition of signal to noise will go to infinity when there is no noise. Our definition prevents this circumstance and normalizes this case to 1 i.e. it is guaranteed that $0<\overline{S / N} \leq 1$.

\section{Closing the Loop}

The loop is closed by feeding the $\overline{S / N}$ into a $5 \mathrm{~Hz}$ low pass filter and then into a summer where the level can be set by the user. The error signal from the summer is used to set the variable gain amplifier which sets the level of the PLL kicker signal sent to the beam.

\section{Minimum Kicker Power}

The minimum power of the kick is determined from measurements (see Figure 18), we find that at $150 \mathrm{GeV}$ the noise power from 1 bunch of coalesced protons on the proton helix $^{\dagger}$ is $-10.3 \mathrm{dBm}$ over $1 \mathrm{kHz}$. The signal to noise ratio $S / N$ between the noise power in this $1 \mathrm{kHz}$ band and the beam response to the coherent kick of the PLL must satisfy the following inequality for a reliable lock

$$
S / N=\left(\frac{P_{s}}{P_{n}}\right) \geq 4
$$

where $P_{s}$ is the power of the kick. If we choose $S / N=4$, then (59) can be solved for $P_{s}$

$$
\left.\begin{array}{rl}
P_{s} & =4 \times P_{n} \\
& =(6-10.3) \mathrm{dBm} \\
& =-3.7 \mathrm{dBm}
\end{array}\right\}
$$

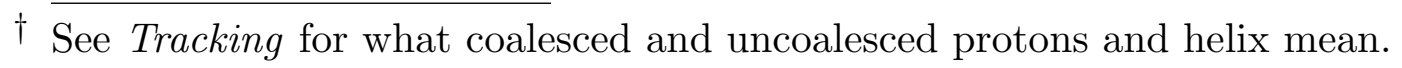


When we kick the beam with $180 \mathrm{~mW}$ of power, we find that the power transferred to the beam is $-3.89 \mathrm{dBm}$ at the kicker sine frequency $27.78 \mathrm{kHz}$ which is about $1 \mathrm{~dB}$ larger than needed. Note that for one uncoalesced bunch, we found that the kicker power can be dramatically reduced from $180 \mathrm{~mW}$ to $7.5 \mathrm{~mW}$ to keep $S / N \approx 4$. We think that this can be explained by going back into the coalescing process which may have introduced much more coherent motion compared to uncoalesced protons. This coherent motion is also rather persistent up the ramp and through the squeeze compared to uncoalesced beam. ${ }^{\dagger}$

$\dagger$ Coherent motion that persists for intense bunches may be interesting physics. See for example ICFA HB2004 conference talks at http://www.gsi.de/search/events/conferences/ICFAHB2004/scientificprogram_e.html 

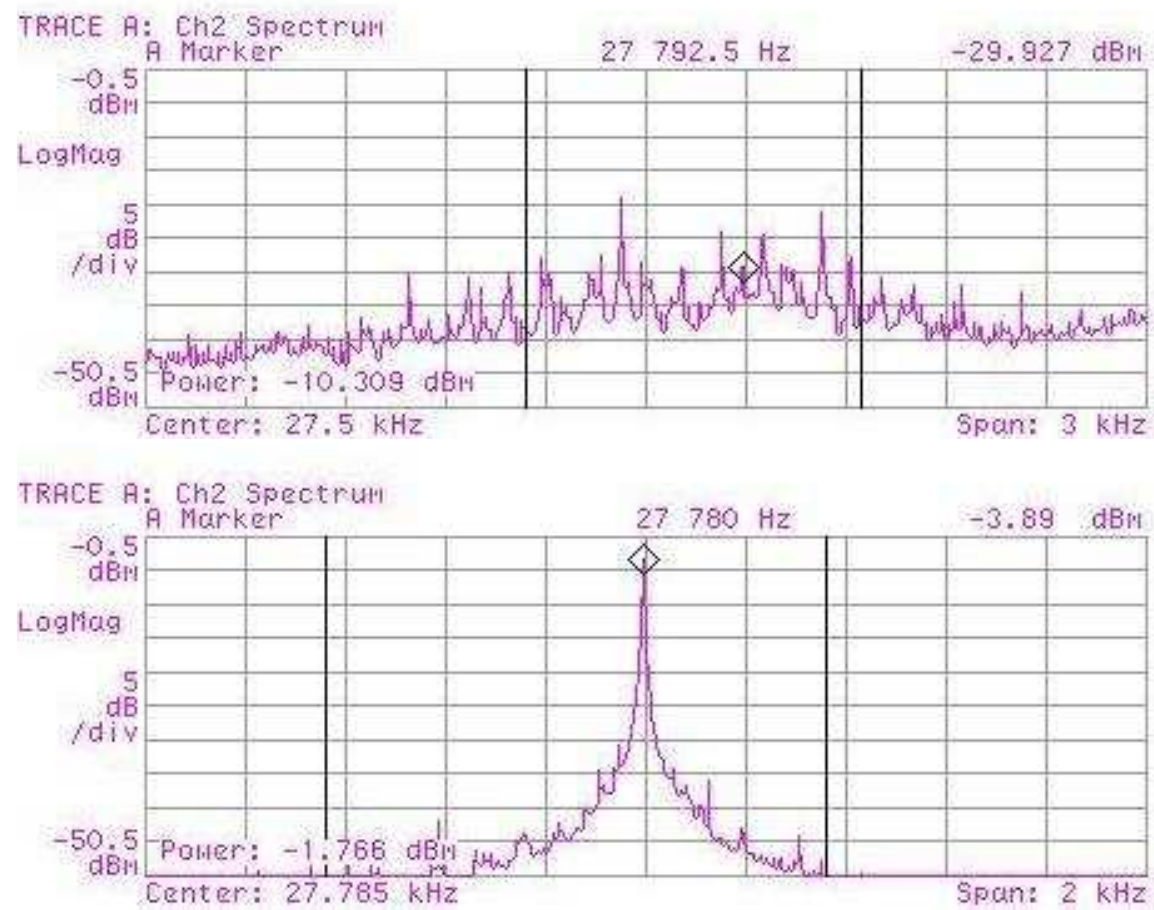

Figure 18 The measured noise power in over $1 \mathrm{kHz}$ which encloses both the horizontal and vertical tunes at $150 \mathrm{GeV}$ for 1 coalesced bunch of protons on the proton helix is shown in the top trace. The effect of kicking the beam with a $180 \mathrm{~mW}$ sine wave is shown in the bottom trace.

\section{SCHOTTKY DETECTOR}

The Schottky detectors used by the PLL tune tracker were built by Martin et $a l^{7}$ in the late 1980s. These detectors are the primary workhorses used routinely for tuning and measuring the betatron tunes in the Tevatron. We will not go into depth on how these detectors work and the reader is referred to the references for the finer details.

A cartoon of one Schottky detector is shown in Figure 19. It is basically a pair of $1 \mathrm{~m}$ 


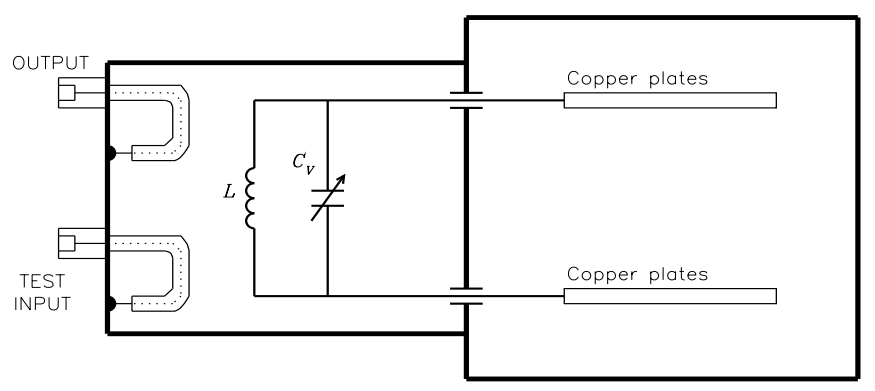

Figure 19 The Schottky detector resonant structure.

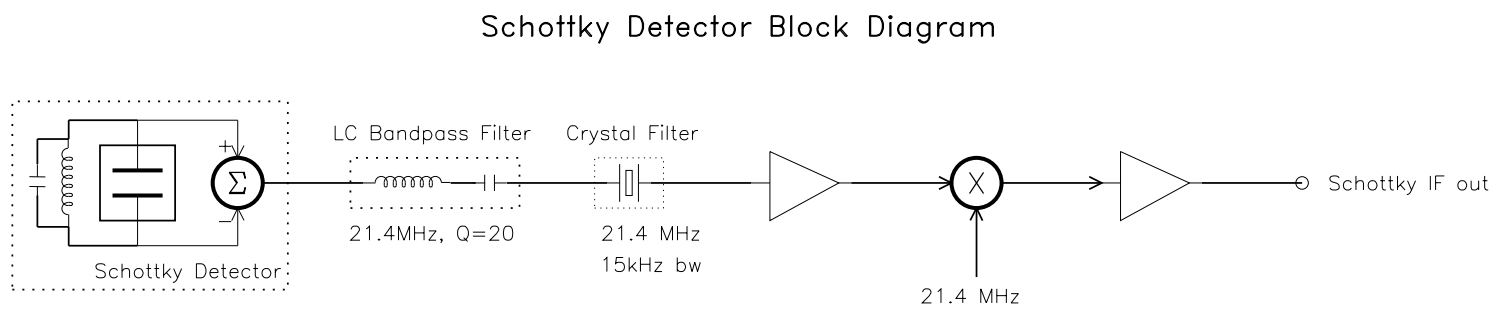

Figure 20 Simplified block diagram of the Schottky electronics.

long striplines contained in a structure with a square cross section. Each stripline consists of a copper bar which can be moved closer or further away from the beam by stepper motors thus changing its sensitivity to the Schottky signal. Its resonant frequency can also be tuned by using the variable capacitor $C_{v}$. Presently these detectors are tuned to resonate around $21.4 \mathrm{MHz}$ with a loaded $Q_{L}=370$. Historically, the resonant frequency was chosen because it is one of the standard IF frequencies (the others being $455 \mathrm{kHz}$ and $10.7 \mathrm{MHz}$ respectively) used in radio communications. Thus unwanted revolution harmonics can be easily filtered out using off-the-shelf narrow band filters. A simplified block diagram of the Schottky electronics is shown in Figure 20. 


\section{AUTOMATIC GAIN CONTROL}

The block diagram of the automatic gain control $^{8}$ (AGC) is shown in Figure 21. The goal of the AGC is to keep the signal to the PLL within its range (which is $\pm 1.2 \mathrm{~V}$ ).

Block Diagram of AGC

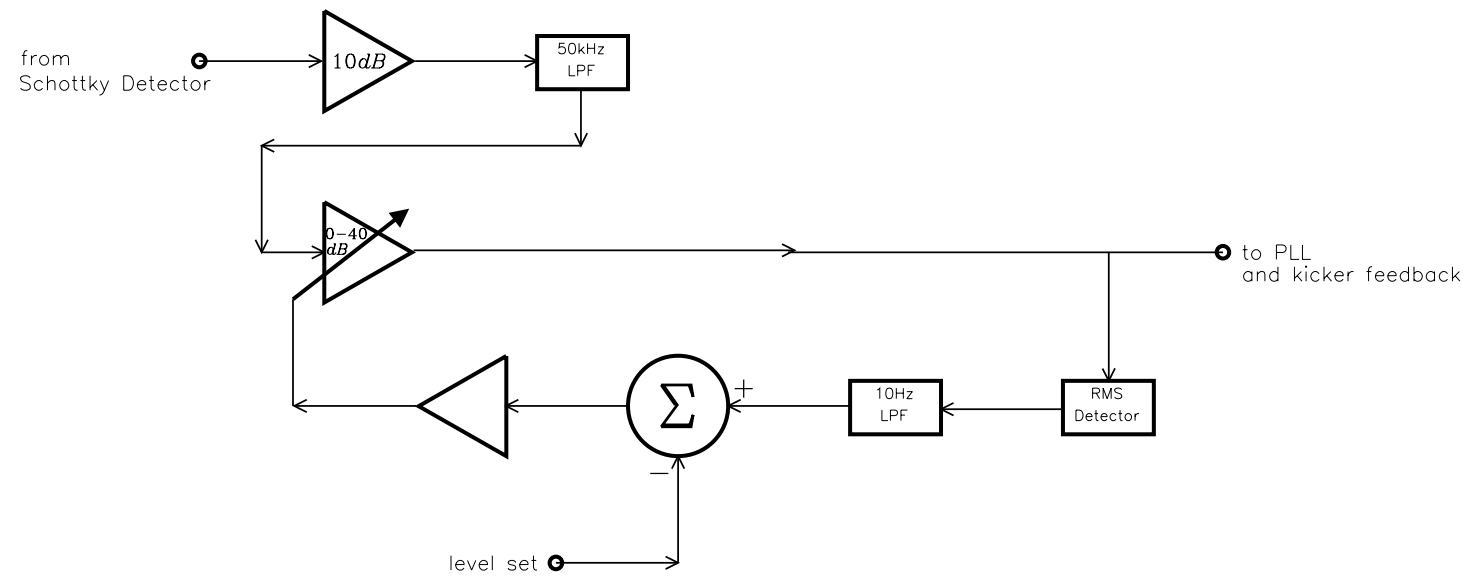

Figure 21 The block diagram of the AGC.

The $10 \mathrm{~Hz}$ low pass filter is to keep fast changes in size of the Schottky detector signal from rapidly changing the gain of the amplifier. This filter prevents a phenomenon called "gain pumping" which is an amplitude modulation of the output. Thus only slow changes which are $<10 \mathrm{~Hz}$ can cause the loop to change the gain of the amplifier. The output signal level is set by the user at the port labelled "level set" in the figure.

One important consideration in designing the AGC is to keep phase shift introduced by the low pass filters to a minimum in the bandwidth of interest. This is to keep the measured phase response of the beam from changing too much in the bandwidth of interest - if for example, the phase shift at resonance is $+10^{\circ}$ then the resonance peak that we measure will be lower than the actual resonance. For the bandwidth from $26 \mathrm{kHz}$ to $29 \mathrm{kHz}$ the 

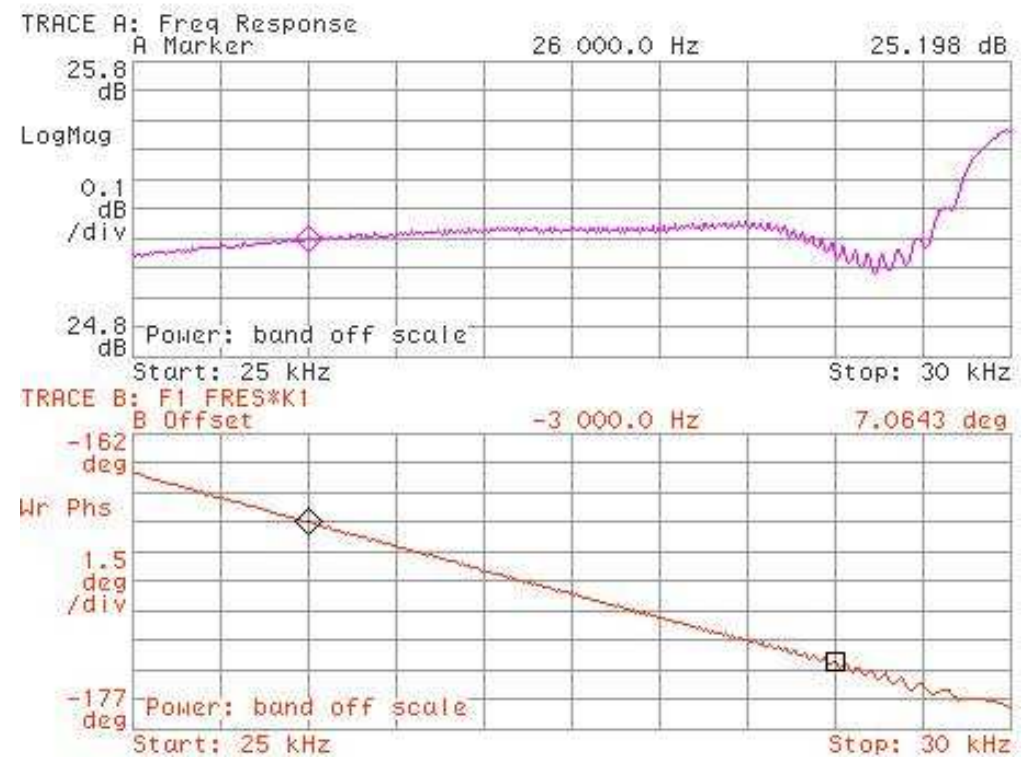

Figure 22 The open loop frequency response of the AGC. The phase shift of $7^{\circ}$ lies between the markers from 26 to $29 \mathrm{kHz}$ which corresponds to 0.544 to 0.61 tune units.

open loop phase shift is shown in Figure 22. And in this case there is a $7^{\circ}$ phase difference between the band edges. The resonance peak reported by the PLL compensates for these phase shifts in software. See section Phase Corrections. 


\section{FIXED DELAY FOR PHASE CORRECTION UP THE RAMP}

If the path length between the two legs into the phase detector is different we will find that when we ramp - because the RF frequency changes, the phase response of the beam will get an additional phase shift $\Delta \theta_{D} \cdot{ }^{9}$ The solution to this problem is to make the path lengths into the phase detector equal so that the output of the phase detector is independent of the RF frequency. First, let us show that there is indeed a phase shift when the RF frequency changes.

Figure 23 shows the relevant parts of the PLL in this discussion. It is an equivalent block diagram of the phase detector, kickers, Schottky pickup, beam response and cables with time delays drawn out explicitly when the PLL is kicking the beam at the betatron frequency $\omega_{\beta}$, i.e. at resonance. The red leg of the phase detector starts from the kicker source then goes into a mixer for up conversion. The phase at $(a)$ is

$$
(a)=\left(\omega_{\beta}+\omega_{\mathrm{osc}}\right) t-\omega_{\mathrm{osc}} \Delta t_{k}
$$

where $-\omega_{\text {osc }} \Delta t_{k}$ comes from the phase shift of the local oscillator after going through a cable of length $\Delta t_{k}$. The negative sign comes from causality.

Next, we go through the equivalent circuit which contains the kicker, beam response, Schottky pickup and electronics, filters and cables. This equivalent circuit has a delay $\Delta t_{s}$ (mainly coming from the Schottky pickup and the crystal filter electronics). Therefore the phase after this is

$$
(b)=\left(\omega_{\beta}+\omega_{\mathrm{osc}}\right) t-\omega_{\mathrm{osc}} \Delta t_{k}-\left(\omega_{\beta}+\omega_{\mathrm{osc}}\right) \Delta t_{s}+\theta_{0}
$$

where $\theta_{0}$ is the phase shift which comes from the beam phase response.

Finally at $(c)$, after down conversion

$$
(c)=\omega_{\beta} t-\left(\omega_{\beta}+\omega_{\mathrm{osc}}\right) \Delta t_{s}-\omega_{\mathrm{osc}}\left(\Delta t_{k}-\Delta t_{p}\right)+\theta_{0} \equiv \theta_{1}
$$




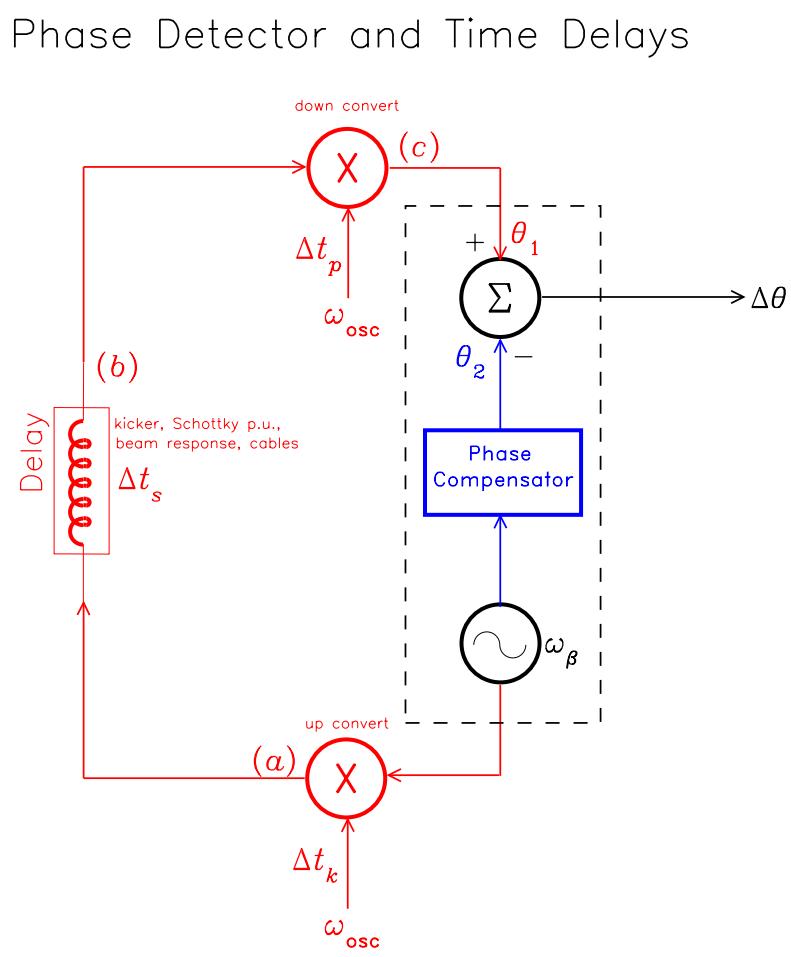

Figure 23 A simplified equivalent circuit of the phase detector, kickers, Schottky pickup, beam response and cables with time delays drawn out explicity.

where $-\omega_{\mathrm{osc}} \Delta t_{p}$ is the phase shift of the local oscillator after going through a cable of length $\Delta t_{p}$.

The blue leg of the phase detector has a phase compensator which we will discuss in the next subsection. Suppose instead that the phase compensator is not present, then

$$
\theta_{2}=\omega_{\beta} t
$$

Note that we do not add in a phase change from the cables which connect the kicker source to the phase detector because the wavelength of the betatron tune $\sim 27.5 \mathrm{kHz}$ is $\sim 10 \mathrm{~km}$.

Finally, the result of the phase $\Delta \theta$ at the output of the phase detector is

$$
\left.\begin{array}{rl}
\Delta \theta & =\theta_{1}-\theta_{2} \\
& =-\omega_{\mathrm{osc}}\left(\Delta t_{k}-\Delta t_{p}+\Delta t_{s}\right)-\omega_{\beta} \Delta t_{s}+\theta_{0}
\end{array}\right\}
$$


When we ramp, the local oscillator frequency changes $\omega_{\text {osc }} \rightarrow \omega_{\text {osc }}^{\prime}$, this means that the output of the phase detector becomes

$$
\Delta \theta^{\prime}=-\omega_{\mathrm{osc}}^{\prime}\left(\Delta t_{k}-\Delta t_{p}+\Delta t_{s}\right)-\omega_{\beta}^{\prime} \Delta t_{s}+\theta_{0}
$$

The phase shift due to the two different local oscillator frequencies is therefore,

$$
\Delta \theta^{\prime}-\Delta \theta=-\left(\omega_{\text {osc }}^{\prime}-\omega_{\text {osc }}\right)\left(\Delta t_{k}-\Delta t_{p}+\Delta t_{s}\right) \equiv \theta_{D}
$$

\section{Example 1}

The measured phase shift of the beam phase response in the Tevatron at $150 \mathrm{GeV}$ and at $980 \mathrm{GeV}$ is $20^{\circ}$ for an oscillator frequency change of $400 \mathrm{~Hz}$. (The actual RF frequency change from $150 \mathrm{GeV}$ to $980 \mathrm{GeV}$ is $53.103 \mathrm{MHz} \rightarrow 53.104 \mathrm{MHz}$, which is $1 \mathrm{kHz}$. However, our oscillator frequency is $21.4 \mathrm{MHz}$ which is $53.1 / 2.5 \mathrm{MHz}$ which means that the frequency change is $1000 / 2.5=400 \mathrm{~Hz}$ ). Using $(67)$, we can calculate the delay in the red leg

$$
\left|\Delta t_{k}-\Delta t_{p}+\Delta t_{s}\right|=\frac{20 \times \pi}{180} \times \frac{1}{2 \pi \times 400}=139 \mu \mathrm{s}
$$

\section{Phase Compensator}

There are at least three methods for building the phase compensator. The first method is obvious and is shown in Figure 24. It requires that we make an equivalent copy of the red leg with the goal that we choose $t_{D}$ so that the red leg and the blue leg have the same path length. The second method comes from (65) and will be discussed in Method II. Finally the method which we have adopted comes from (67) and will be discussed in Method III. 


\section{Phase Compensator}

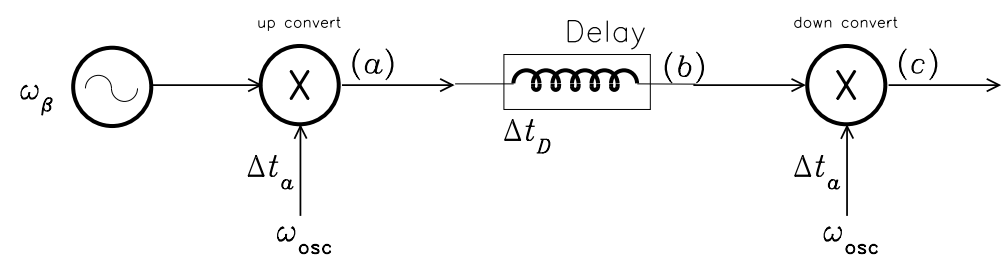

Figure 24 The phase compensator circuit which is an equivalent copy of the red leg in Figure 23.

\section{Method I}

The phase at each point in Figure 24 is

$$
\begin{aligned}
& (a)=\left(\omega_{\beta}+\omega_{\mathrm{osc}}\right) t-\omega_{\mathrm{osc}} \Delta t_{a} \\
& (b)=\left(\omega_{\beta}+\omega_{\mathrm{osc}}\right) t-\omega_{\mathrm{osc}} \Delta t_{a}-\left(\omega_{\beta}+\omega_{\mathrm{osc}}\right) \Delta t_{D} \\
& (c)=\omega_{\beta} t-\left(\omega_{\beta}+\omega_{\mathrm{osc}}\right) \Delta t_{D} \equiv \theta_{2}
\end{aligned}
$$

where the choice of $t_{D}$ is the time delay determined by the following:

$$
\begin{aligned}
\Delta \theta & =\theta_{1}-\theta_{2} \\
& =-\omega_{\mathrm{osc}}\left(\Delta t_{k}-\Delta t_{p}+\Delta t_{s}\right)-\omega_{\beta} \Delta t_{s}+\left(\omega_{\beta}+\omega_{\mathrm{osc}}\right) \Delta t_{D}+\theta_{0} \\
& =-\omega_{\beta}\left(\Delta t_{s}-\Delta t_{D}\right)+\theta_{0} \\
& =\omega_{\beta}\left(\Delta t_{p}-\Delta t_{k}\right)+\theta_{0} \\
& \approx \theta_{0}
\end{aligned}
$$

if we choose $\Delta t_{D}=\Delta t_{k}-\Delta t_{p}+\Delta t_{s}$ then $\Delta \theta$ just reflects the phase response of the beam at resonance. The term $\omega_{\beta}\left(\Delta t_{p}-\Delta t_{k}\right)$ is small because even if the difference in oscillator cable lengths between the mixers is $\sim 100$ ns this term contributes only about $1^{\circ}$ because $\omega_{\beta} \sim 2 \pi \times 27.5 \times 10^{3} \mathrm{~s}^{-1}$. In reality the difference cable length is $\ll 100 \mathrm{~ns}$. Therefore, (70) is independent of the oscillator frequency $\omega_{\mathrm{osc}}$ and only depends on time delays. 


\section{Method II}

The second method is similar to Method I except in its choice of placement of the delay. We notice that $\Delta t_{p}$ is opposite in sign to $\Delta t_{k}$ and $\Delta t_{s}$ in (65). Therefore, if we add delay to the down convert local oscillator, we can have the situation where $\Delta t_{p} \rightarrow \Delta t_{k}+\Delta t_{p}$ and thus the phase contribution from the oscillator in (65) becomes identically zero. The phase that is left behind $-\omega_{\beta} \Delta t_{s}$ is easily compensated by adding a digital delay in the Phase Compensator box shown in Figure 23. The digital delay block diagram is shown in Figure 25.

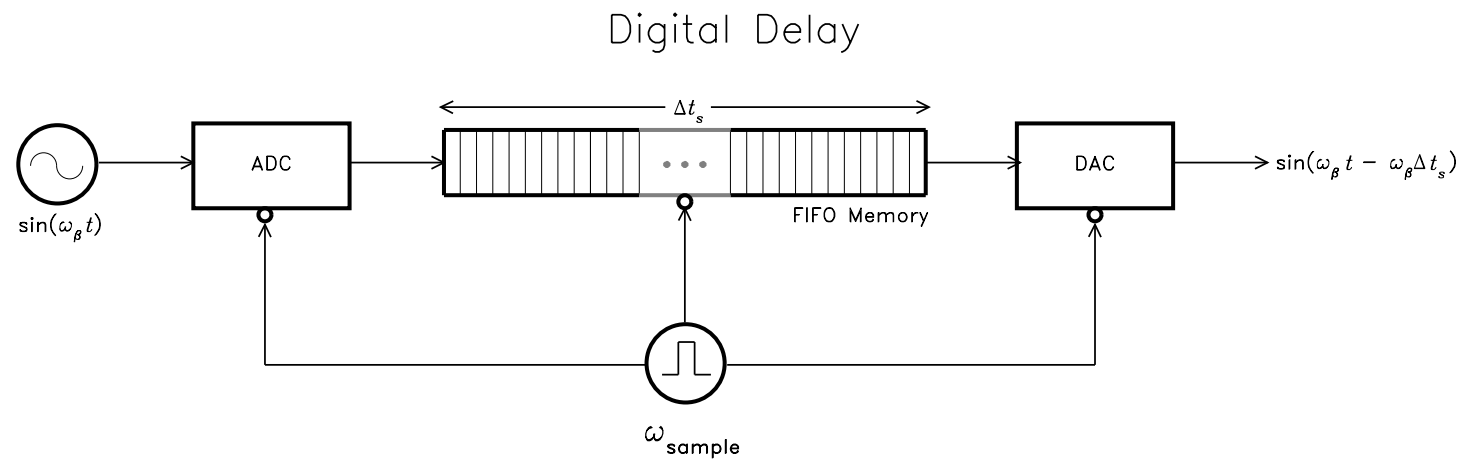

Figure 25 A digital delay with an ADC frontend, FIFO memory and DAC backend.

The ADC frontend samples the input kicker sine wave and then stuffs the result into FIFO memory at a rate given by the sampling clock which runs independently of the low level RF. After shifting through memory for $\Delta t_{s} \mathrm{~s}$, the output of the FIFO is sent to a DAC which results in a time delayed sine wave.

\section{Method III}

The main problem with Method I and Method II is the design of the fixed $139 \mu \mathrm{s}$ 
delay — clearly, in practice, cables cannot be used. To do this digitally like in Figure 25, $\omega_{\text {sample }}$ must be at a minimum $4 \times \omega_{\text {osc }} \approx 2 \pi \times 100 \times 10^{6} \mathrm{~s}^{-1}$ in both methods and that still does not guarantee that we will not be overwhelmed with phase noise because current 14 bit ADCs are limited to sampling frequencies of $<150 \mathrm{MHz}$, i.e. this design can be done but is a challenge. A much better way is to look at (67) and notice that if we introduce a frequency dependent phase shifter in the up convert leg ${ }^{\dagger}$ of the local oscillator we can remove the need for the high speed ADC. We start at $(a)$ of Figure 26

$$
(a)=\left(\omega_{\beta}+\omega_{\mathrm{osc}}\right) t-\omega_{\mathrm{osc}} \Delta t_{k}+\Delta \phi\left(\omega_{\mathrm{osc}}\right)
$$

and continuing through the other points

$$
\begin{aligned}
& (b)=\left(\omega_{\beta}+\omega_{\mathrm{osc}}\right) t-\omega_{\mathrm{osc}} \Delta t_{k}-\left(\omega_{\beta}+\omega_{\mathrm{osc}}\right) \Delta t_{s}+\theta_{0}+\Delta \phi\left(\omega_{\mathrm{osc}}\right) \\
& (c)=\omega_{\beta} t-\left(\omega_{\beta}+\omega_{\mathrm{osc}}\right) \Delta t_{s}-\omega_{\mathrm{osc}}\left(\Delta t_{k}-\Delta t_{p}\right)+\theta_{0}+\Delta \phi\left(\omega_{\mathrm{osc}}\right) \equiv \theta_{1} \\
& \Delta \theta=-\omega_{\mathrm{osc}}\left(\Delta t_{k}-\Delta t_{p}+\Delta t_{s}\right)-\omega_{\beta}\left(\Delta t_{s}-\Delta t_{d}\right)+\theta_{0}+\Delta \phi\left(\omega_{\mathrm{osc}}\right)
\end{aligned}
$$

When the oscillator frequency changes, $\omega_{\text {osc }} \rightarrow \omega_{\text {osc }}^{\prime}$ the phase detector output is

$$
\Delta \theta^{\prime}=-\omega_{\mathrm{osc}}^{\prime}\left(\Delta t_{k}-\Delta t_{p}+\Delta t_{s}\right)-\omega_{\beta}^{\prime}\left(\Delta t_{s}-\Delta t_{d}\right)+\theta_{0}+\Delta \phi\left(\omega_{\mathrm{osc}}^{\prime}\right)
$$

If we choose $\Delta t_{d}=\Delta t_{s}$ in the phase compensator box in Figure 26, the phase shift due to the two different local oscillator frequencies is

$$
\Delta \theta^{\prime}-\Delta \theta=-\left(\omega_{\mathrm{osc}}-\omega_{\mathrm{osc}}^{\prime}\right)\left(\Delta t_{k}-\Delta t_{p}+\Delta t_{s}\right)+\Delta \phi\left(\omega_{\mathrm{osc}}^{\prime}\right)-\Delta \phi\left(\omega_{\mathrm{osc}}\right) \equiv \Delta \theta_{D}
$$

To remove any dependence of $\Delta \theta$ on $\omega_{\text {osc }}$ clearly, we want $\Delta \theta_{D} \equiv 0$. This means that

$$
\Delta \phi\left(\omega_{\mathrm{OSc}}^{\prime}\right)-\Delta \phi\left(\omega_{\mathrm{osc}}\right)=\left(\omega_{\mathrm{osc}}-\omega_{\mathrm{OSc}}^{\prime}\right) \Delta t_{D}
$$

where $t_{D}=\Delta t_{k}-\Delta t_{p}+\Delta t_{s}$. If we choose $\Delta \phi\left(\omega_{\text {osc }}\right)=0$, then we have the following linear relationship between the phase shift and the local oscillator frequency

$$
\Delta \phi\left(\omega_{\mathrm{OSC}}^{\prime}\right)=-\left(\omega_{\mathrm{OSC}}^{\prime}-\omega_{\mathrm{osc}}\right) \Delta t_{D}
$$

$\dagger$ Note this analysis is also valid if we stick the phase shifter in the down convert leg. 


\section{Phase Detector and Time Delays}

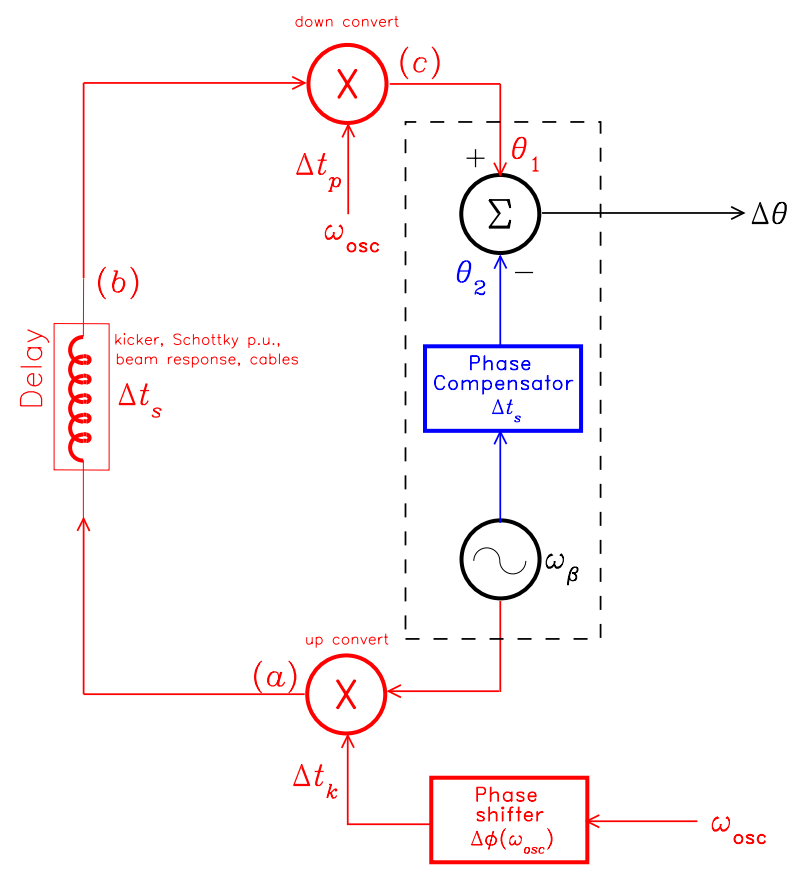

Figure 26 We add in a phase shifter in the local oscillator leg of the up convert mixer and a delay $\Delta t_{d}=\Delta t_{s}$ in the phase compensator box.

Using this relationship, the phase detector will be independent of the local oscillator frequency and hence the ramp frequency.

In the Tevatron, the RF frequency varies proportionately with the ramp current. Thus by measuring the ramp current, we can set the amount of phase shift that will compensate for the ramp. See Figure 27.

\section{Example 2}

Continuing from Example 1, at $150 \mathrm{GeV}$, the phase shifter will be set so that it shifts the local oscillator phase by zero degrees. At $980 \mathrm{GeV}$, the phase shifter will shift the oscillator phase using $(76)$ by $-2 \pi \times 400 \times\left(139 \times 10^{-6}\right)=-20^{\circ}$ in order to keep the 

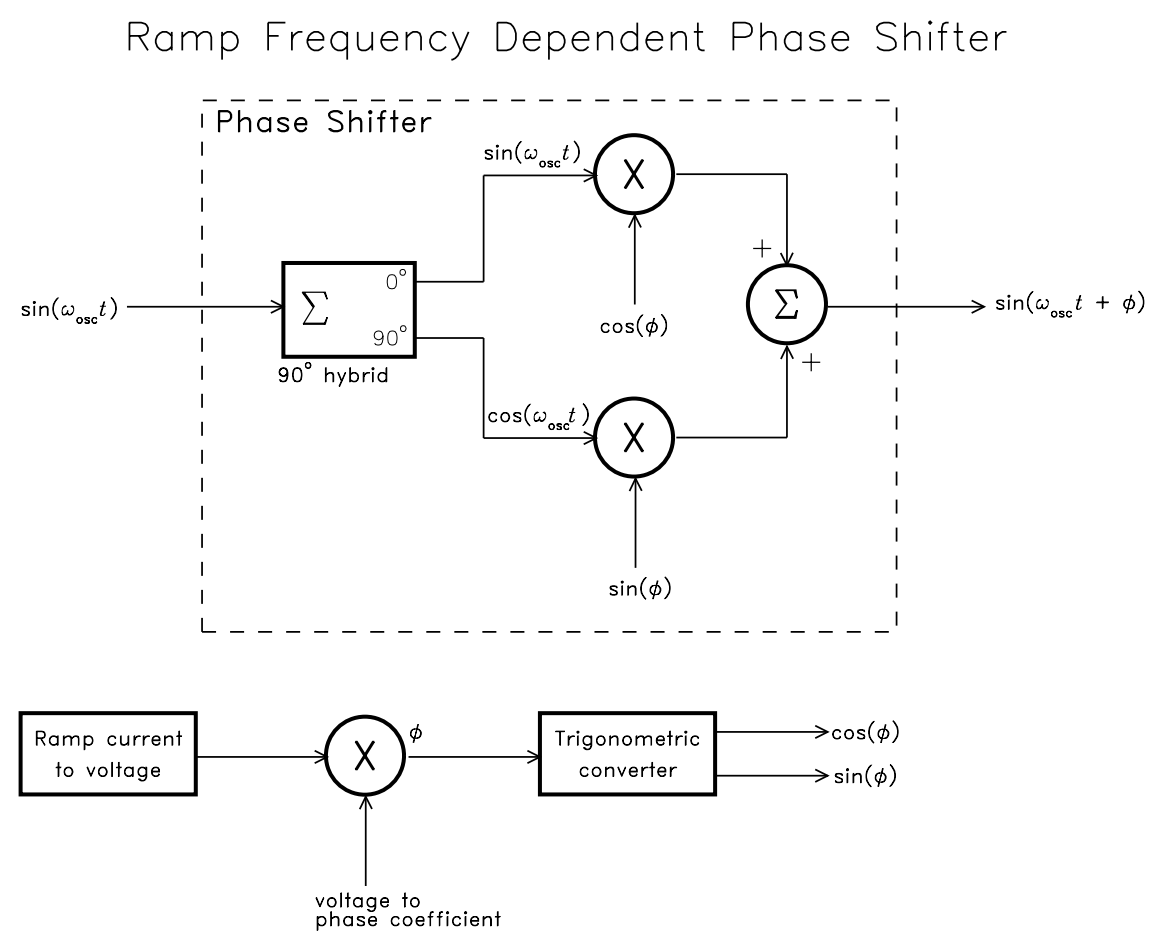

Figure 27 The ramp frequency dependent phase shifter.

phase detector independent of the local oscillator frequency change. 
Measurements 


\section{MEASUREMENTS}

In this section, we will discuss all the measurements that we have made.

(i) The closed loop response of the PLL. This measurement uses the idea that when we have a step change in the resonant frequency of the beam, we can use it to derive the closed loop frequency response $\mathcal{H}$.

(ii) Linear corrections to the reported locked tunes. As was previously mentioned in the Automatic Gain Control section, the filters used in the system will cause small phase shifts that we need to correct.

(iii) The response of the beam with different excitations at $150 \mathrm{GeV}$. We will show the spectra of 1 bunch of coalesced protons on the proton helix when it is kicked continuously and pulsed. The phase response measured by the PLL in these cases is also discussed.

(iv) PLL tracking up the ramp and through the squeeze with different beam conditions. This is, of course, the entire goal of the project. We will show the PLL tracking performatince in four different sets of conditions up the ramp and through the squeeze.

$(v)$ Emittance growth of the beam. The Achilles heel of this method is emittance growth. We have to keep the emittance growth down to acceptable levels without compromising the signal to noise ratio discussed in PLL Parameters from Noise Characteristics. 


\section{CLOSED LOOP RESPONSE OF THE CONTINUOUS PLL}

A practical method for measuring the closed loop frequency response of the PLL parameters will be discussed here. We will not use actual beam for this measurement but instead use the "fake beam response circuit" shown in Appendix I. This circuit allows us to get an idea of what the closed loop response is without using actual beam. There are two reasons for doing this

(i) In real life, access to the Tevatron beam for beam studies is difficult.

(ii) Using the fake beam response circuit allows us to control exactly the conditions of the measurements.

Note that the closed loop frequency response depends on the frequency response of the beam. Clearly from $(7), \mathcal{H}$ depends on the beam phase response slope $K$. Thus the measurements done here only give us $\mathcal{H}$ for one fixed value of $K$. From the frequency response of the fake beam shown in Figure 28 we find that $K=0.6 \times 10^{-2} \mathrm{~s}$ which from Table 1 , is within the range of $K$ for the actual beam.

Theory

We will derive a method where we can measure the closed loop response of the PLL with a digital oscilloscope. ${ }^{10}$ From (3), the relationship between the excitation phase $\theta_{2}$ and the beam phase $\theta_{0}$ is

$$
\theta_{2}=\mathcal{H} \theta_{0}
$$

If there is a step in the beam resonant frequency i.e. $\theta_{0} \rightarrow \theta_{0}+\Delta \omega_{0} / s^{2}$ then the response 

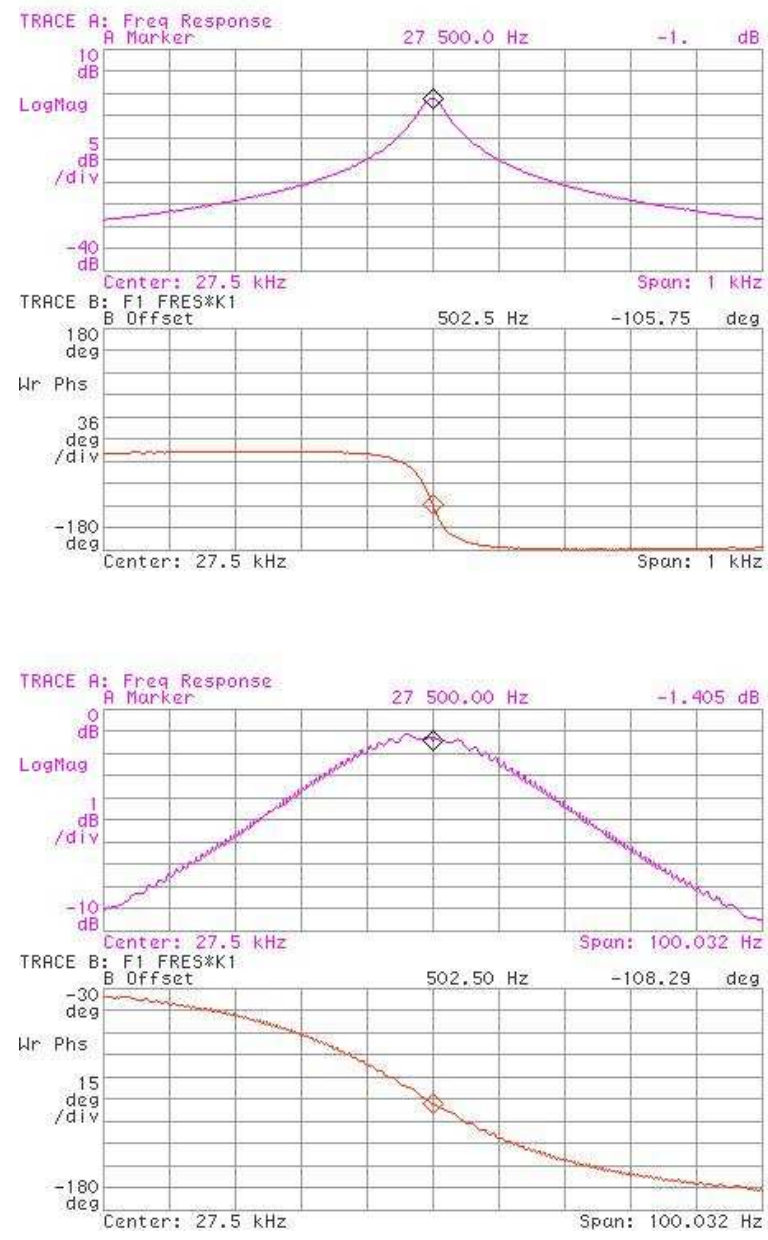

Figure 28 The frequency response of the fake beam circuit. Top picture is with a $1 \mathrm{kHz}$ span and bottom picture is with a $100 \mathrm{~Hz}$ span.

of the PLL excitation $\theta_{2} \rightarrow \theta_{2}+\Delta \theta_{2}$

$$
\left.\begin{array}{rl}
\theta_{2}+\Delta \theta_{2} & =\mathcal{H}\left(\theta_{0}+\frac{\Delta \omega_{0}}{s^{2}}\right) \\
\Rightarrow \quad \Delta \theta_{2} & =\mathcal{H} \frac{\Delta \omega_{0}}{s^{2}}
\end{array}\right\}
$$


Multiplying (78) by $s$, and defining $s \Delta \theta_{2} \equiv \Delta \omega_{2}(s)$, we have

$$
\Delta \omega_{2}(s)=\mathcal{H} \frac{\Delta \omega_{0}}{s}
$$

i.e. we have moved to a relationship between frequencies rather than phases. Therefore, if we differentiate once the change in excitation frequency in the time domain after we step the beam resonant frequency by $\Delta \omega_{0}$, we get

$$
s \Delta \omega_{2}(s)=\mathcal{H} \Delta \omega_{0}
$$

Thus the method becomes clear: to measure $\mathcal{H}$, we need to change the frequency of the beam resonance by a step $\Delta \omega_{0}$, differentiate the PLL excitation frequency that tries to follow this step and then Fourier transform it to obtain $\mathcal{H}$.

\section{Setup and Results}

The setup to measure the closed loop response of the PLL is shown in Figure 29. The frequency source which is used to clock the commutating filter is set up to do frequency shift key (FSK) modulation. And because of the way our commutating filter is implemented, if we want the fake beam to resonate at $f \mathrm{kHz}$, we must clock it at $8 \times f \mathrm{kHz}$. Thus, for the fake beam to start at a resonant frequency of $27.500 \mathrm{kHz}$ and then have a $37.5 \mathrm{~Hz}$ step and repeat every $10 \mathrm{~s}$, the FSK carrier is set to $(8 \times 27.5000) \mathrm{kHz}$, hop frequency to $(8 \times 27.5375) \mathrm{kHz}$ and periodic frequency of $100 \mathrm{mHz}$. This step causes the PLL to change its kicker frequency $\omega_{k}$ in order to follow this step. The results are shown in Figure 30(a) for the cases when the gain multiplier is set to 1, 2, 5, 7 and 10. We differentiate (a) once to get the results shown in Figure 30(b). And finally Fourier transforming it, we get the closed loop frequency response shown in Figure 30(c).

From Figure 30(c), we can see that the natural frequency $\omega_{n}$ and damping factor $\zeta$ of the PLL are dependent on the gain multiplier $A$. These results should be compared to 


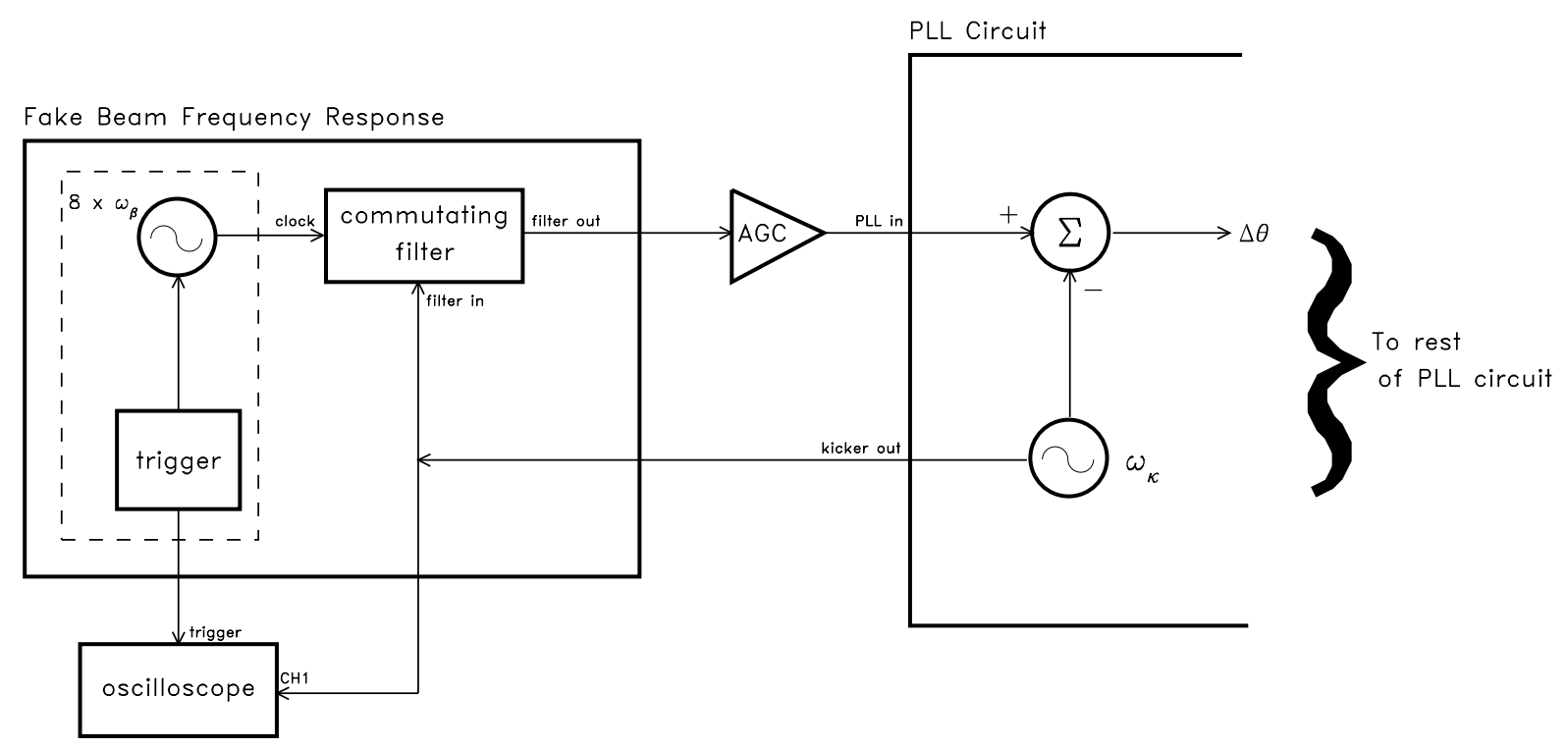

Figure 29 The block diagram of the setup used to measure the closed loop frequency response of the PLL.

Figure 4 and the calculations presented in the section Calculating $G(s)$. We expected that $\omega_{n}=(2 \pi \times 3.5) \mathrm{s}^{-1}$ when $\zeta=0.7$ and $A=3$ from theory but from these measurements, we found that when $A=2$, the measured results are $\zeta=0.7, \omega_{n}=(2 \pi \times 2.2) \mathrm{s}^{-1}$. Table 2 summarizes the measurements for other values of $A$.

\begin{tabular}{c|c|c|c}
\hline \multicolumn{4}{c}{ Table 2. Measured $\omega_{3 \mathrm{~dB}}, \boldsymbol{\omega}_{n}$ and $\boldsymbol{\zeta}$} \\
\hline $\boldsymbol{A}$ & $\boldsymbol{\omega}_{3 \mathrm{~dB}} \times 2 \pi\left(\mathrm{s}^{-1}\right)$ & $\boldsymbol{\omega}_{n} \times 2 \pi\left(\mathrm{s}^{-1}\right)$ & $\boldsymbol{\zeta}$ \\
1 & 1.3 & 1.7 & 0.93 \\
2 & 2.5 & 2.2 & 0.71 \\
5 & 6.2 & 4.7 & 0.34 \\
7 & 7.6 & 6.0 & 0.28 \\
10 & 9.4 & 7.5 & 0.19 \\
\hline \hline
\end{tabular}



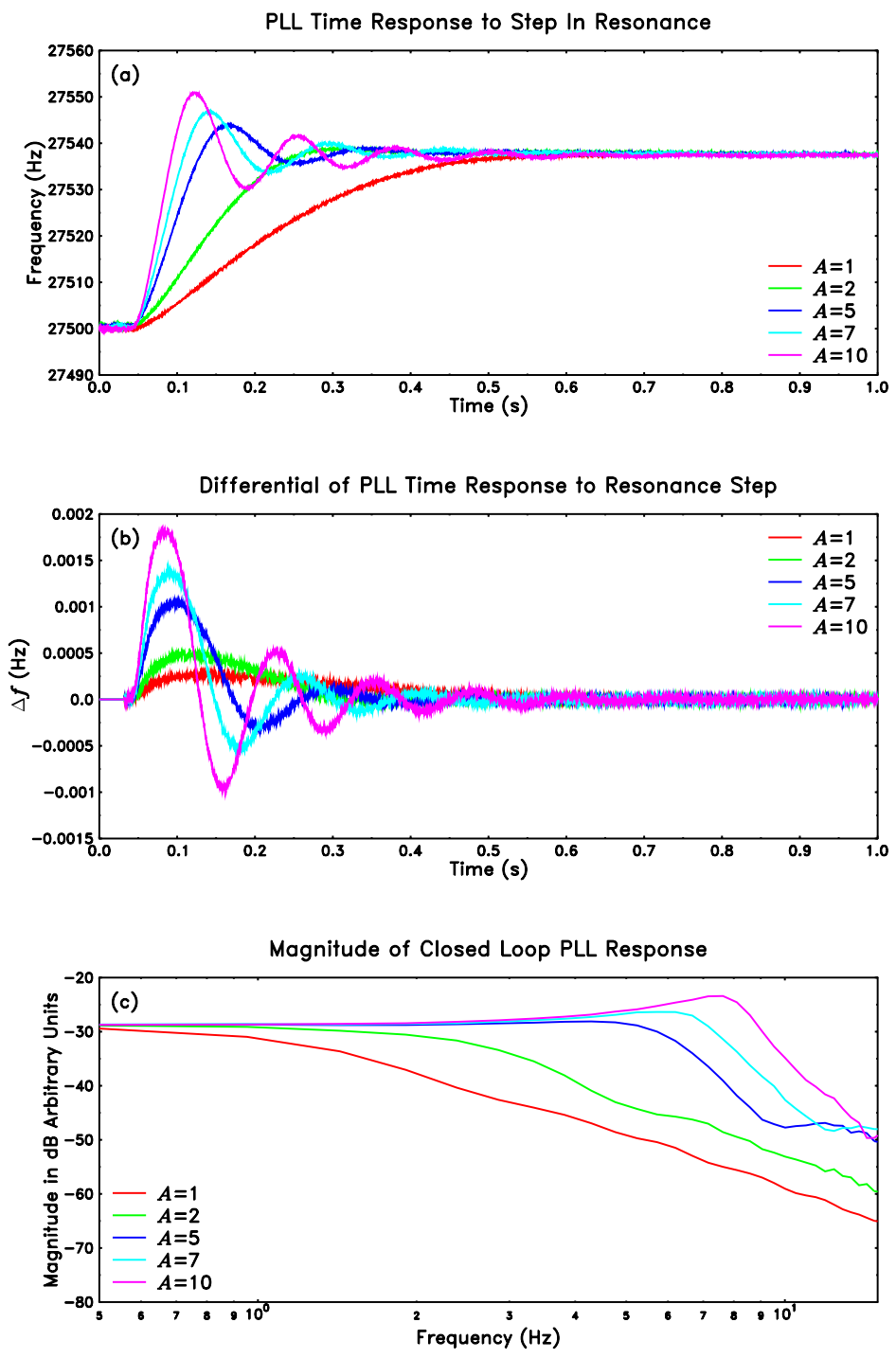

Figure 30 The magnitude of the frequency response of the PLL using the method discussed in Closed Loop Response of the PLL for different gain multipliers $A$. 


\section{PHASE CORRECTIONS}

We need to perform small phase corrections in software because the filters used in the PLL introduce phase shifts, for example in the AGC. See Figure 22. These corrections are not related to the phase shift from change in the RF frequency that was discussed in Fixed Delay for Phase Correction Up the Ramp but come from the excitation sampling different parts of the filters in the PLL circuit.

The corrections are found with the fake beam frequency response circuit because we know exactly where the the fake beam resonance is supposed to be. First, we set the fake beam resonance to $27.500 \mathrm{kHz}$, and then we set the phase offset of the PLL so that it returns exactly $27.500 \mathrm{kHz}$. The fake beam resonance is then moved and the lock frequency $f_{m}$ found by the PLL is plotted against this. See Figure 31 . The linear fit gives the correction for the phase shifts from filters which are

$$
\begin{array}{ll}
f_{t}=1.00465 \times f_{m}-0.127999 & \text { for continuous PLL } \\
f_{t}=1.01673 \times f_{m}-0.459365 & \text { for pulsed PLL }
\end{array}
$$

where $f_{t}$ is the true resonance frequency and $f_{m}$ is the measured PLL lock frequency. Interestingly the slopes are nearly one in both cases, but the intercepts are clearly different. 
PLL Correction for Continuous PLL

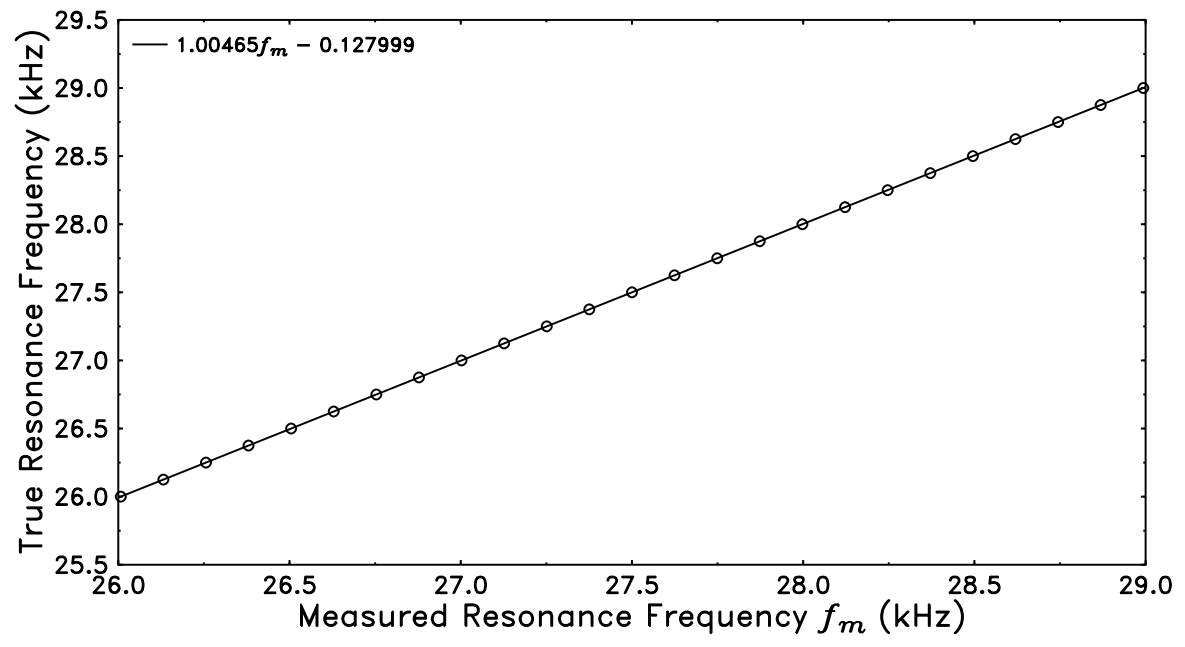

PLL Correction for Pulsed PLL

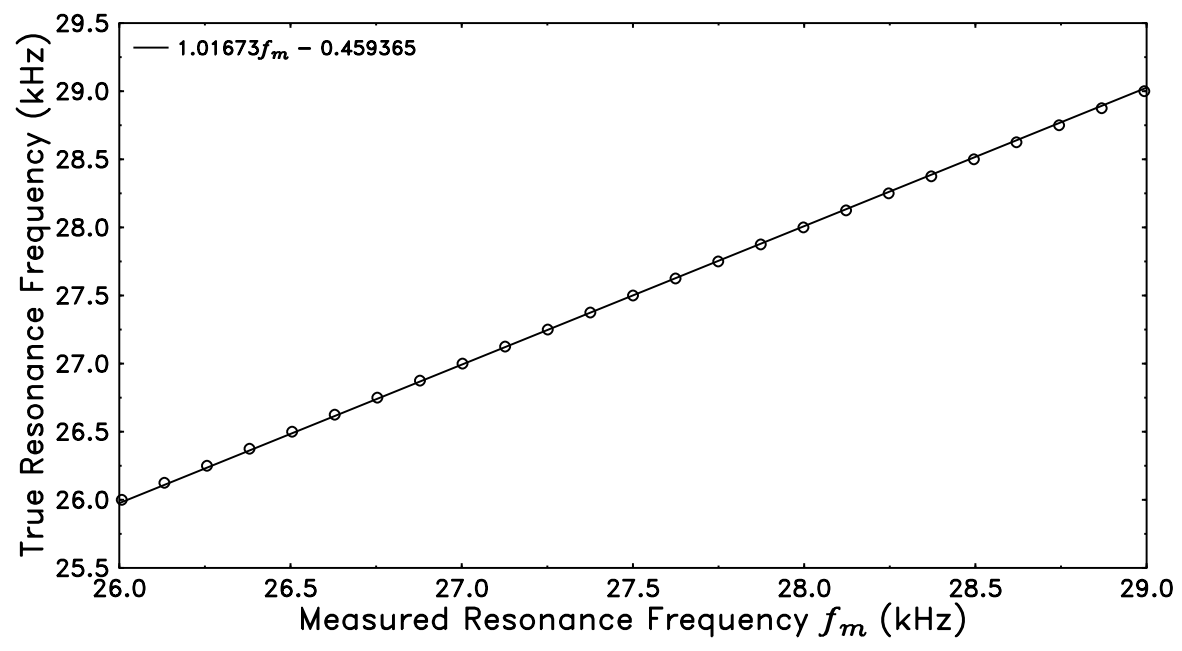

Figure 31 Corrections to the frequency for both continuous and pulsed PLL. 


\section{BEAM RESPONSE FROM EXCITATION}

The behaviour of 1 coalesced bunch of protons on the proton helix at $150 \mathrm{GeV}$ with chromaticity set to about 4 units when excited continuously or pulsed is shown here. The Schottky signal of this bunch when it is not excited is shown in Figure 32(a). The kicker power level is set to $180 \mathrm{~mW}$ which is the minimum power needed to be $6 \mathrm{~dB}$ above noise in $1 \mathrm{kHz}$ of bandwidth (from section Minimum Kicker Power) for continuous excitation. The spectrum when this is applied to the beam is shown in Figure 32(c). The spectrum when the beam is pulse excited (10 ms on and $30 \mathrm{~ms}$ off) is shown in Figure 32(b). Not surprisingly, the beam is less excited with a $25 \%$ duty cycle compared to $100 \%$ duty cycle. The phase response of the beam when continuously excited and pulsed excited is shown in Figure 32(d). Pulsed excitation clearly smooths out the phase response.

Since the pulsed excitation clearly smooths out the synchrotron lines, we want to see whether the pulsed PLL will indeed lock close to the middle of the betatron line. We start with both PLLs locked to the centre of the betatron tune. Then we yank the PLL excitation

frequency by $+350 \mathrm{~Hz}$ (3.5 synchrotron lines) and $-500 \mathrm{~Hz}$ (6 synchroton lines) to cause the PLL to lose lock. After a while, the PLL will eventually lock to some synchrotron line for the continuous PLL while for the pulsed PLL it locks to a place that is close to the centre of the betatron tune. Figure 33 shows the result of this experiment. It is interesting that this effect is not symmetric about the centre. This is easily understood by looking at the phase response shown in Figure 32(d). For the continuous PLL, we use the red curve. We see that when the initial lock is at $27.7 \mathrm{kHz}$ and after we yank the excitation frequency to $28.05 \mathrm{kHz}$, there is only one nice zero crossing to the right of $27.7 \mathrm{kHz}$. While yanking to lower frequencies, there are no zero crossings other than the one at $27.7 \mathrm{kHz}$. The same argument applies for the pulsed PLL. 
Date: 09-06-02 Time: 09: 10 AM

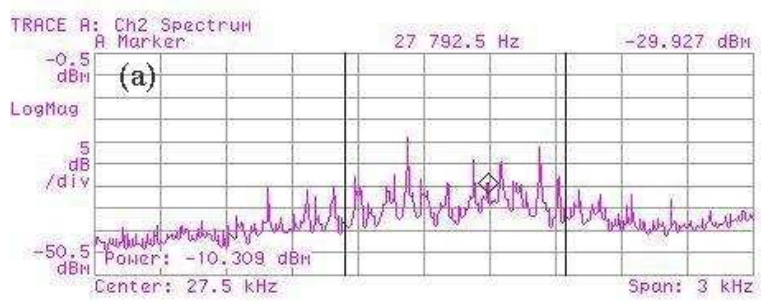

Date: 09-06-02 Time: 07:37 AM

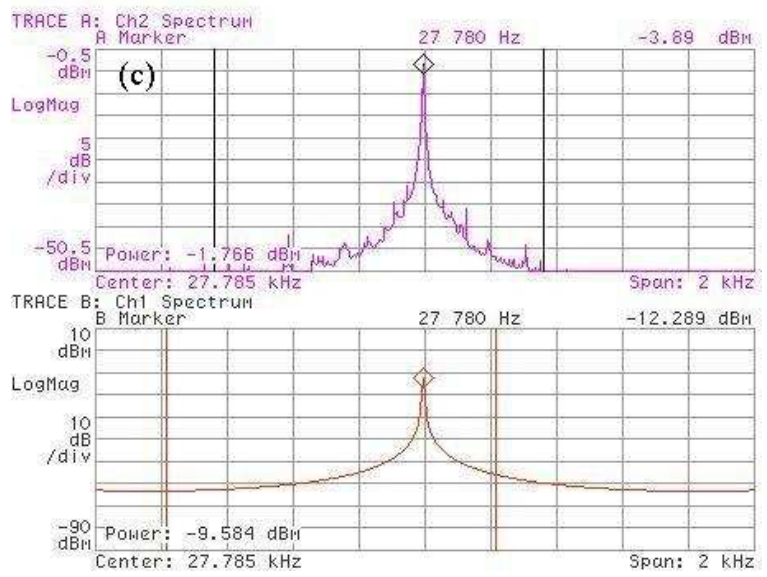

Date: 09-06-02 Time: 07:35 AM

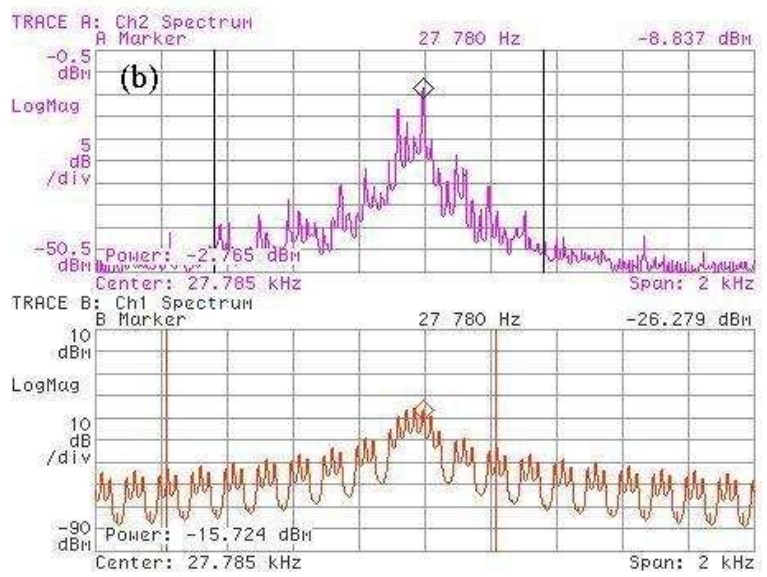

Phase Response of Coalesced Bunch at $150 \mathrm{GeV}$

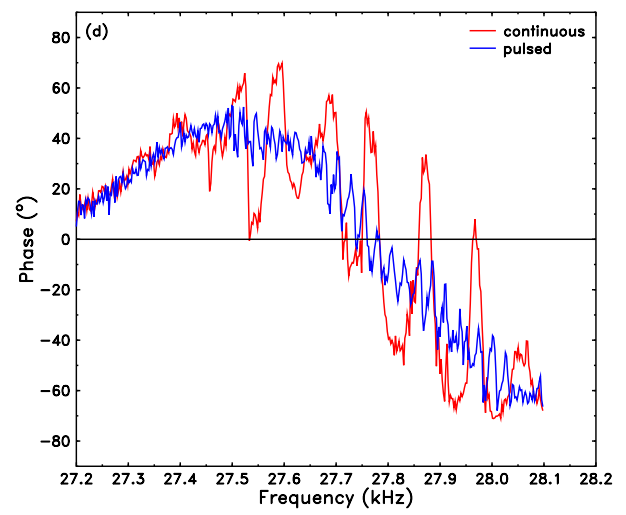

Figure 32 These figures show the spectra of one coalesced protron on the proton helix for different kick conditions. Figures (a), (b) and (c) are the spectra measured by the VSA. Trace A (purple) is the spectrum of the beam signal from the AGC with the kicker off. Trace B (red) is the spectrum of the kicker signal before up conversion. (a) shows the case when the kicker is off. (b) is when the kicker is pulse kicking the beam. (c) is when the kicker is continuously kicking th beam. (d) is the phase response for the case when the beam is continuously kicked (red) and pulse kicked (blue). 


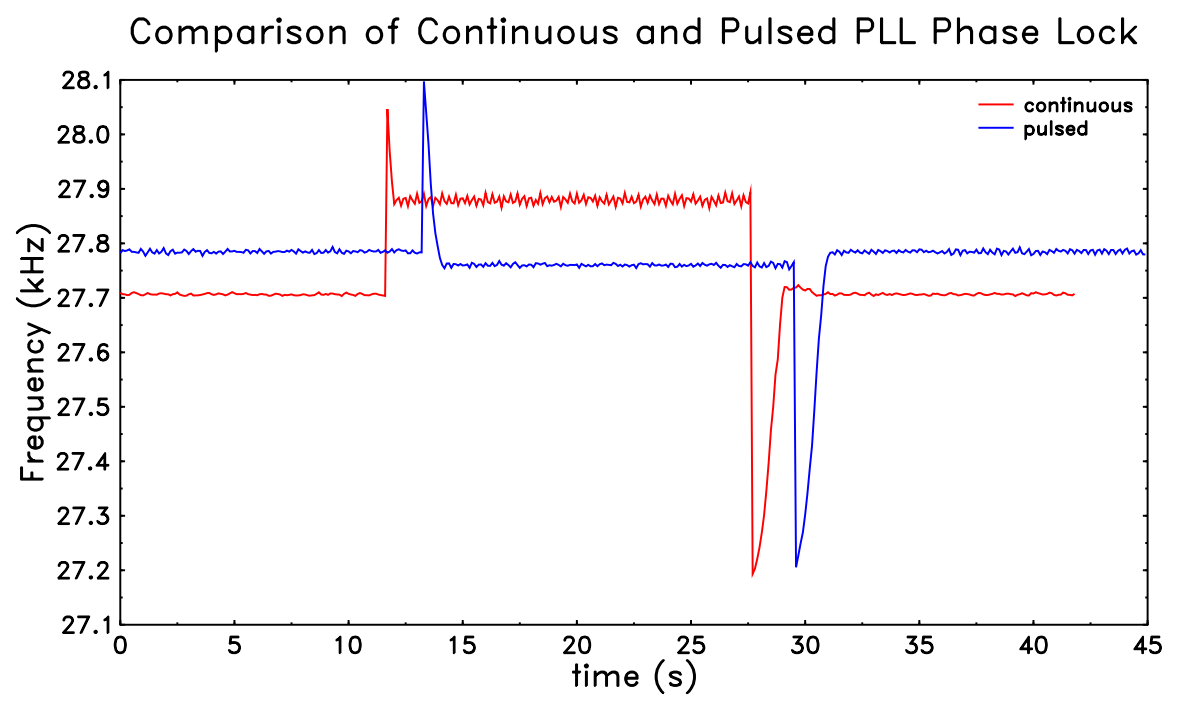

Figure 33 This compares the continuous PLL and pulsed PLL when the lock frequency is yanked by $+300 \mathrm{~Hz}$ and $-500 \mathrm{~Hz}$. Clearly the continuous PLL locks to different synchrotron lines while the pulsed PLL locks to a frequency that is close to the centre of the betatron tune.

\section{TRACKING}

Finally, we get to the results which show that the tune tracker PLL does track the tunes up the ramp and through the squeeze. The Tevatron has two beam conditions:

(i) Uncoalesced beam is when we have $\sim 200 \times 10^{9}$ protons spread out equally in 30 adjacent buckets. This is the usual Tevatron diagnostics beam condition.

(ii) Coalesced beam is when we have $\sim 200 \times 10^{9}$ protons all in one bucket. This is the HEP beam condition for 1 proton bunch. (For HEP, the Tevatron runs with 36 coalesced bunches).

and three electrostatic separator conditions:

(i) Separators off. This means that the helix is off and thus the beam is on the central 
orbit.

(ii) Separators on, in the usual polarity. This means that the protons are on the proton helix and this is the condition during HEP. Separators in this polarity are used to separate the protons and the anti-protons which are in the same beam tube.

(iii) Separators on, opposite polarity. This means that the protons are on the pbar helix. This is only used for beam studies.

For this paper, we will only track the tunes up the ramp and through the squeeze with the following conditions:

(i) Uncoalesced beam, central orbit, with continuous PLL. See Figure 35 and Figure 36.

(ii) Uncoalesced beam, central orbit, with pulsed PLL. See Figure 37 and Figure 38.

(iii) Coalesced beam, proton helix, with continuous PLL. See Figure 39 and Figure 40.

(iv) Coalesced beam, proton helix, with pulsed PLL. See Figure 41 and Figure 42.

Note that the tune tracker did not have all its parts at the time the experiments were performed. The missing parts are the kicker feedback circuit and the phase compensator circuit. Without the kicker feedback circuit, we just kept the kicker power fixed up the ramp and through the squeeze. The absence of the phase compensator is a little bit more of a concern. As we had discussed in Fixed Delay for Phase Correction Up the Ramp, there is a phase shift of $20^{\circ}$ between $150 \mathrm{GeV}$ and $980 \mathrm{GeV}$. This phase shift is sufficient to cause the PLL to smoothly slip between synchrotron lines. Although we did not lose lock in any of the cases we discuss below, the PLL frequency locks to a lower frequency than the actual betatron frequency as the beam is ramped. 


\section{Uncoalesced Protons, Central Orbit, Continuous PLL}

Using continuous excitation on uncoalesced protons on the central orbit, we excite the beam with $7.5 \mathrm{~mW}$ of power with the gain multiplier set to 1 . Figure 35(a) shows how the excitation behaves up the ramp. It is clear that the excitation is strong enough to overwhelm the Schottky noise of the beam and thus we cannot tell whether the PLL is actually tracking anything real. Thus, to avoid being misled, we compared the locked frequency collected here with a store of uncoalesced protons (store 3624) that did not have the PLL turned on. By superimposing the locked frequency data and the waterfall plot from store 3624, we get Figure 35(b). We can see that the white trace which is the locked frequency data does follow the features of the Schottky spectra of store 3624. Going through the squeeze, we again have to superimpose the PLL data onto store 3624 . See Figure 36(b). We see that the white trace tracks through the squeeze spectra rather well.

\section{Uncoalesced Protons, Central Orbit, Pulsed PLL}

For the pulsed PLL, we have the kicker turned on for $10 \mathrm{~ms}$ and off for $30 \mathrm{~ms}$. The kicker power is still at $7.5 \mathrm{~mW}$ while the gain multiplier has been increased to 5 . Clearly looking at Figure 37(a), the PLL locked frequency tracks the Schottky data up the ramp rather well. Comparing the noise levels of the frequency lock with continuous PLL and pulsed PLL, we see that pulsed PLL is at least twice as noisy as continuous PLL. However, because the gain multiplier is different, this is not a fair comparison.

\section{Coalesced Protons, Proton Helix, Continuous PLL}

For coalesced protons on the proton helix, we have to kick the beam with $180 \mathrm{~mW}$ of power in order to satisfy the $S / N \geq 4$ requirement. The gain multiplier is set to 
4. Coalesced protons on the proton helix is the condition that is closest to HEP - the differences are

(i) there is only 1 bunch compared with 36 bunches used for HEP.

(ii) We do not have anti-protons in the study.

Looking at Figure 39(a), we see that the white trace follows HEP store 3699 up the ramp rather well until maybe at $500 \mathrm{GeV}$. This is where we may have departed from the true betatron tune. There are two possibilities:

(i) the anti-protons have shifted the horizontal tune up in the HEP store compared to the PLL store which did not have anti-protons.

(ii) the phase shift from RF change has not been corrected.

Another interesting observation is that the noise of the lock increases as the ramp energy increases. This is clearly seen in the bottom graph of Figure 39, at $300 \mathrm{~s}$ the gain multiplier is reduced from 4 units to 2 units and immediately the noise level went down.

With the gain multiplier set to 2 units and at the same power level, we go through the squeeze. See Figure 40. Tracking through the squeeze produces a much better result. Note that the white trace is stuck to the left of the Schottky spectra because we did not change the phase offset by $20^{\circ}$.

\section{Coalesced Protons, Proton Helix, Pulsed PLL}

For the pulsed PLL, we turn the excitation on for $10 \mathrm{~ms}$ and off for $30 \mathrm{~ms}$. The gain multiplier is set to 16. Although, we see that the white trace follows the Schottky data of store 3699 up the ramp is a lot more noisy when compared to the continuous PLL. In fact, when we turn the gain multiplier down at $980 \mathrm{GeV}$ from 16 to 1 the noise level immediately goes down. The bottom graph of Figure 41 shows this effect at $250 \mathrm{~s}$. Going 


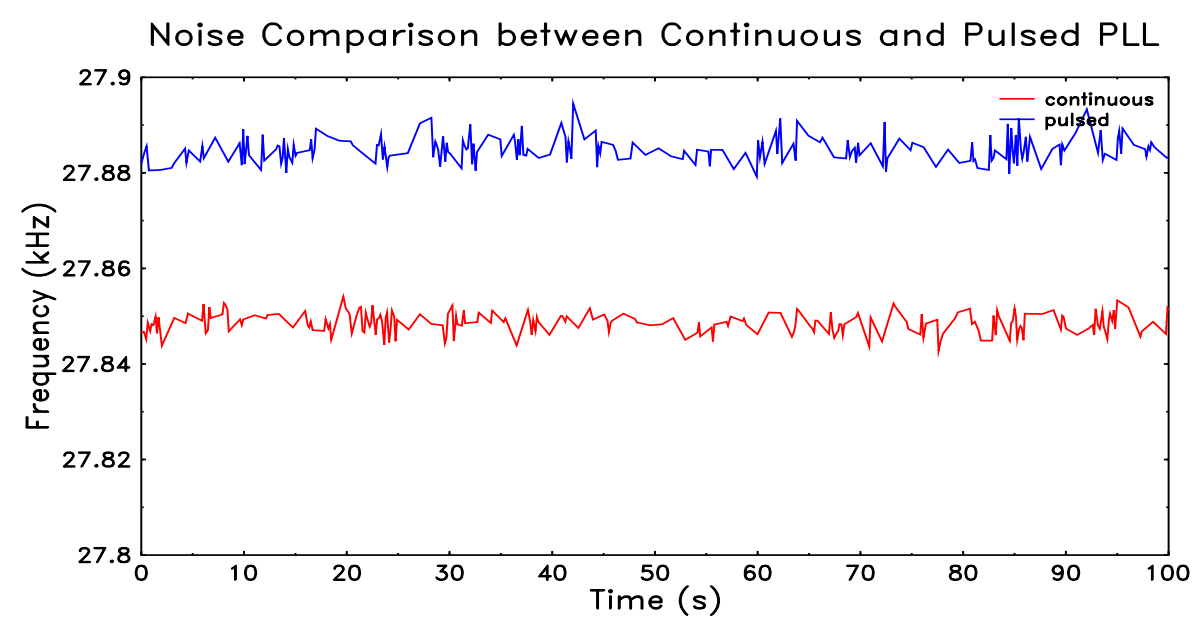

Figure 34 The noise comparision between continuous and pulsed PLL. Gain multiplier is set to 4 for continuous PLL and 16 for pulsed PLL.

through the squeeze with the gain multiplier set to 1 the noise level of the pulse PLL is comparable to the continuous PLL.

\section{Comparison between Continuous and Pulsed PLL}

We can plot the tracking performance of the continuous and pulsed PLL and compare them. From empirical observation, it is always necessary that we run the pulsed PLL at a higher gain than the continuous PLL, which means that the pulsed PLL will always be noisier than the continuous PLL. However, because the optimum gain for either PLLs has not been determined yet, the final verdict on the exact noise difference between the methods has not been settled. We will, nonetheless, venture at a stab at the difference and guess that the noise level is less than a factor of 2 between the two methods after the gain has been optimized. For example, see Figure 34, where we have zoomed in at $150 \mathrm{GeV}$ for coalesced protons on the proton helix. The rms error of the tune is $2 \mathrm{~Hz}$ (or $4 \times 10^{-5}$ tune units) for continuous PLL while for pulsed PLL it is $3 \mathrm{~Hz}$ (or $6 \times 10^{-5}$ tune units). 

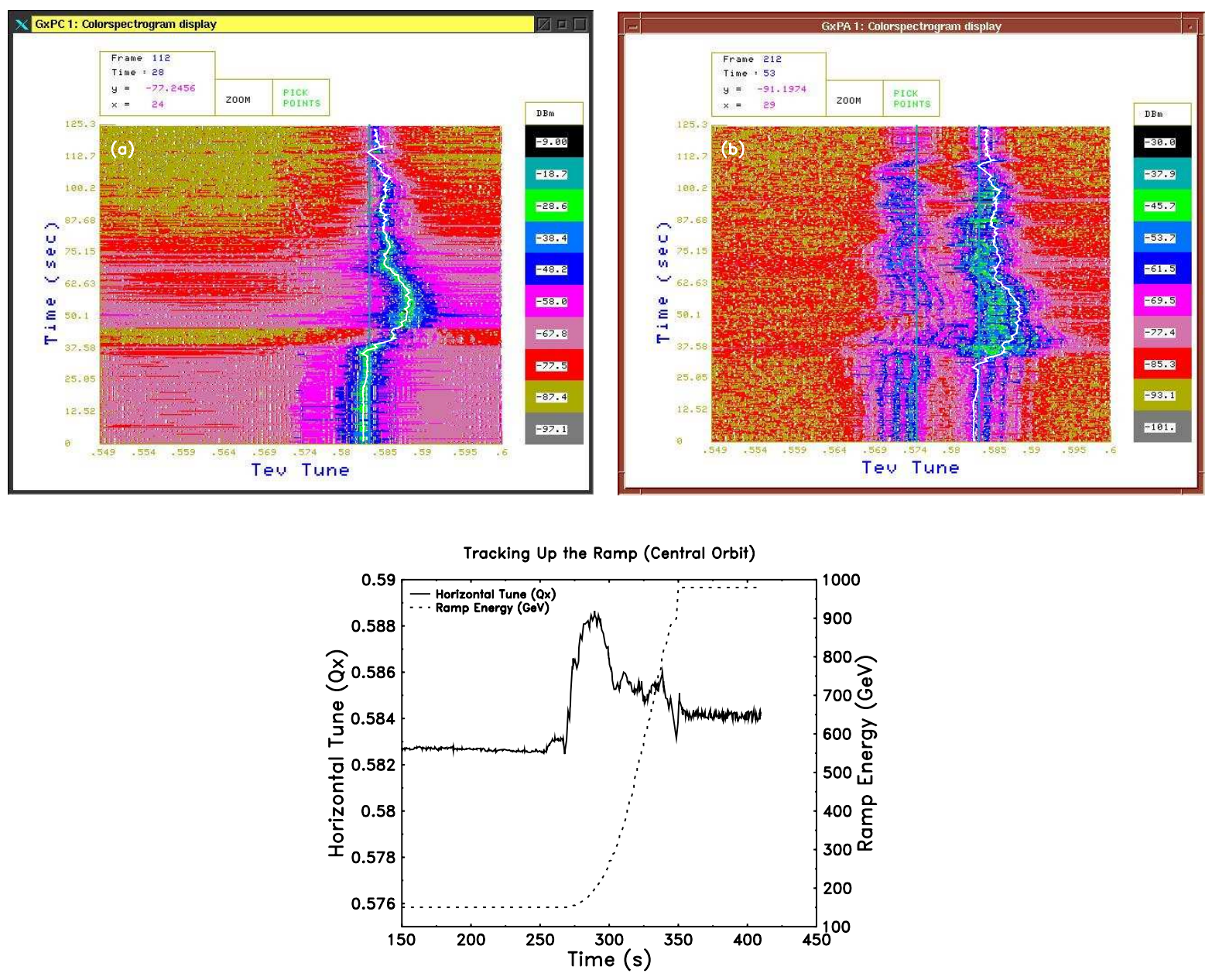

Figure 35 Tracking using continuous PLL up the ramp on the central orbit with uncoalesced beam. Picture (a) is the waterfall spectrum of the Schottky signal with the PLL kicker on. The white trace superimposed in this picture is the PLL track data. Picture (b) is the central orbit waterfall spectrum of store number 3624 without the PLL going up the ramp. Superimposed on this is the data from (a). Finally, the bottom picture is the data from (a) plotted against the Tevatron energy ramp. 

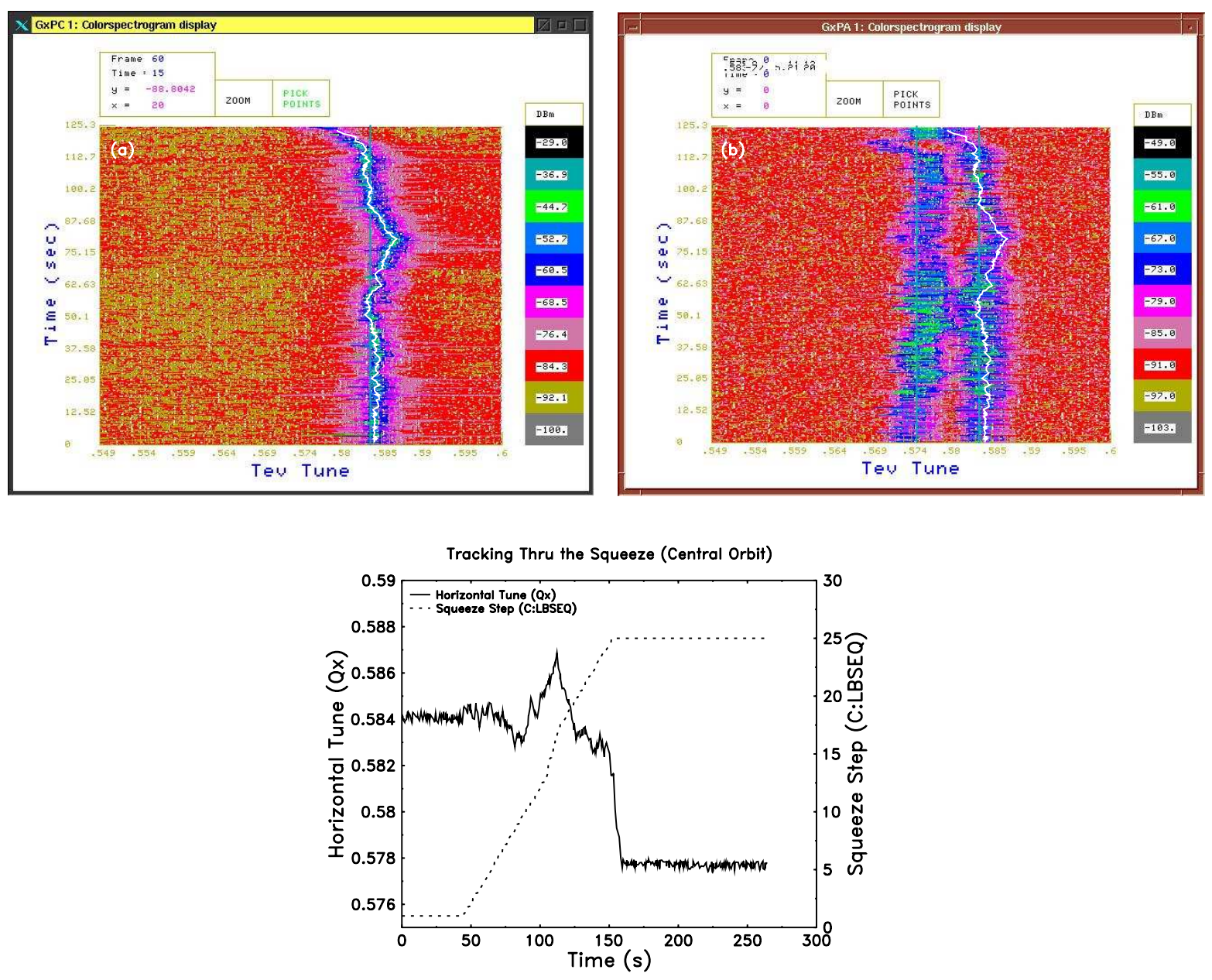

Figure 36 Tracking using continuous PLL through the squeeze on the central orbit with uncoalesced beam. Picture (a) is a waterfall spectrum of the Schottky signal with the PLL kicker on. The white trace superimposed in this picture is the PLL track data. Picture (b) is the central orbit waterfall spectrum of store number 3624 through the squeeze without the PLL. Superimposed on this is the data from (a). Finally, the bottom picture is the data from (a) plotted against the Tevatron squeeze. 

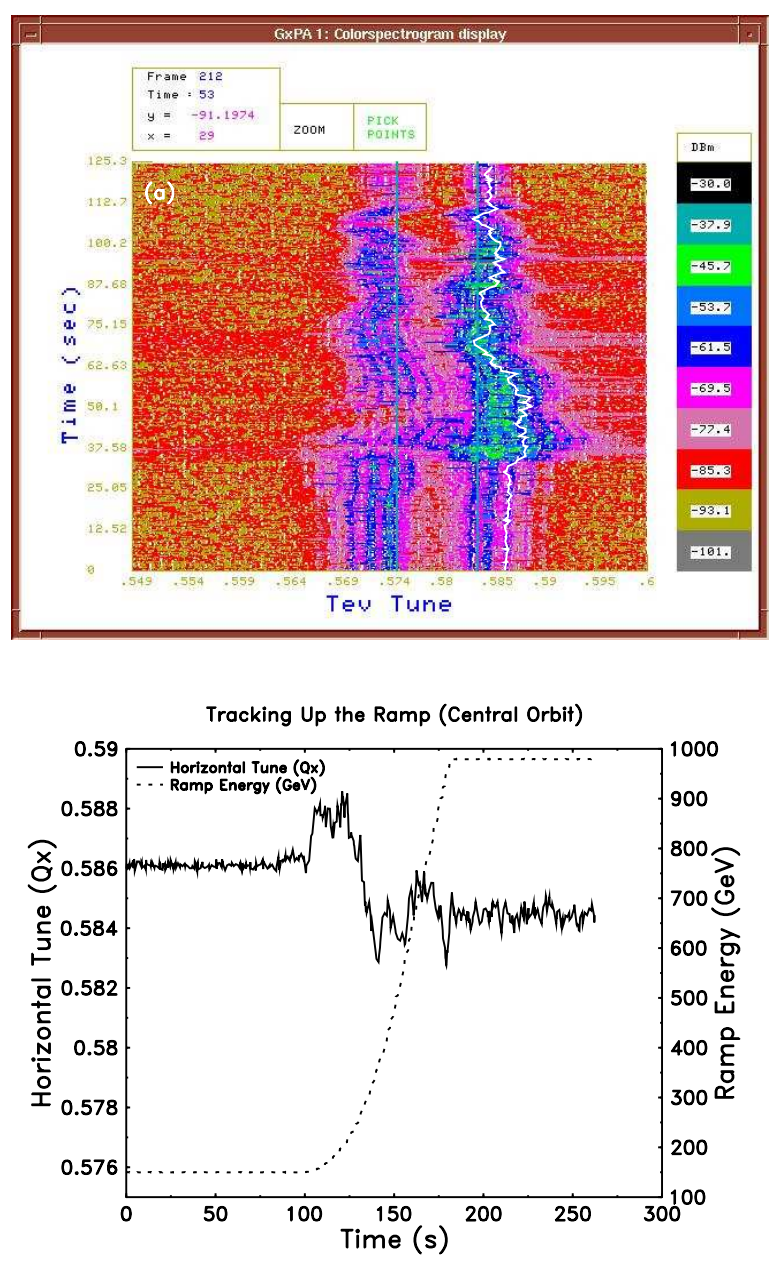

Figure 37 Tracking using pulsed PLL up the ramp on the central orbit with uncoalesced beam. Picture (a) is the central orbit waterfall spectrum of store number 3624 without the PLL going up the ramp. Superimposed on this is the PLL locked frequency data from another store. Finally, the bottom picture is the same PLL tune data plotted against the Tevatron energy ramp. 

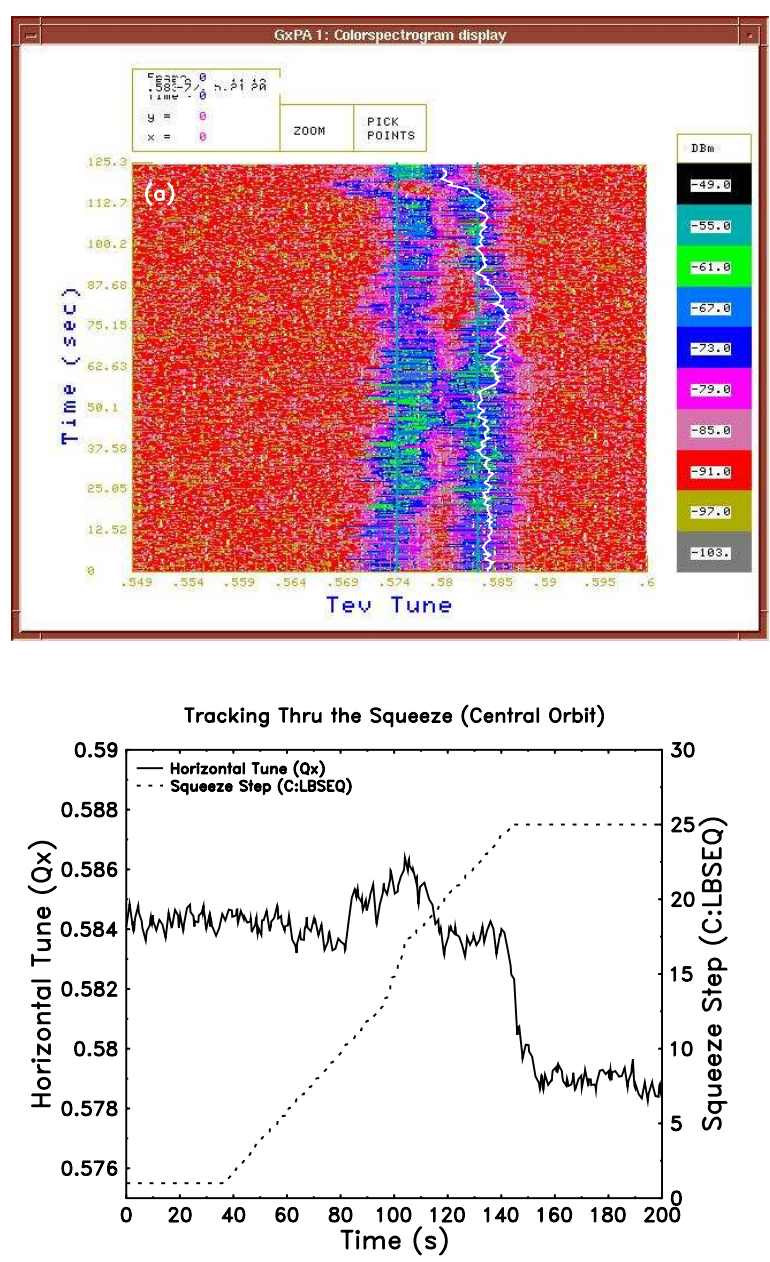

Figure 38 Tracking using pulsed PLL through the squeeze on the central orbit with uncoalesced beam. Picture (a) is the central orbit waterfall spectrum of store number 3624 without the PLL going through the squeeze. Superimposed on this is the PLL locked frequency data data from another store. Finally, the bottom picture is the same PLL tune data plotted against the Tevatron squeeze. 

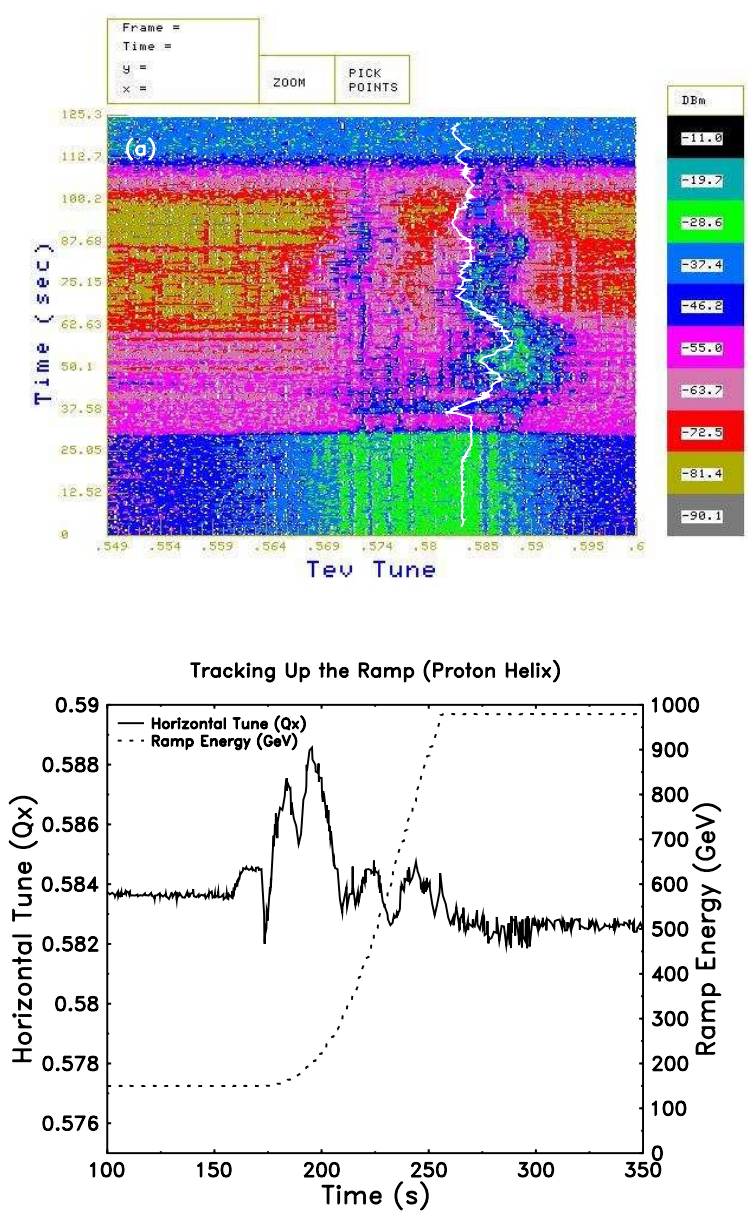

Figure 39 Tracking using continuous PLL up the ramp on the proton helix with one bunch of coalesced protons. Picture (a) is the proton helix waterfall spectrum of store number 3699 without the PLL going up the ramp. Superimposed on this is the PLL locked frequency from another store. Finally, the bottom picture is the same PLL tune data plotted against the Tevatron energy ramp. 

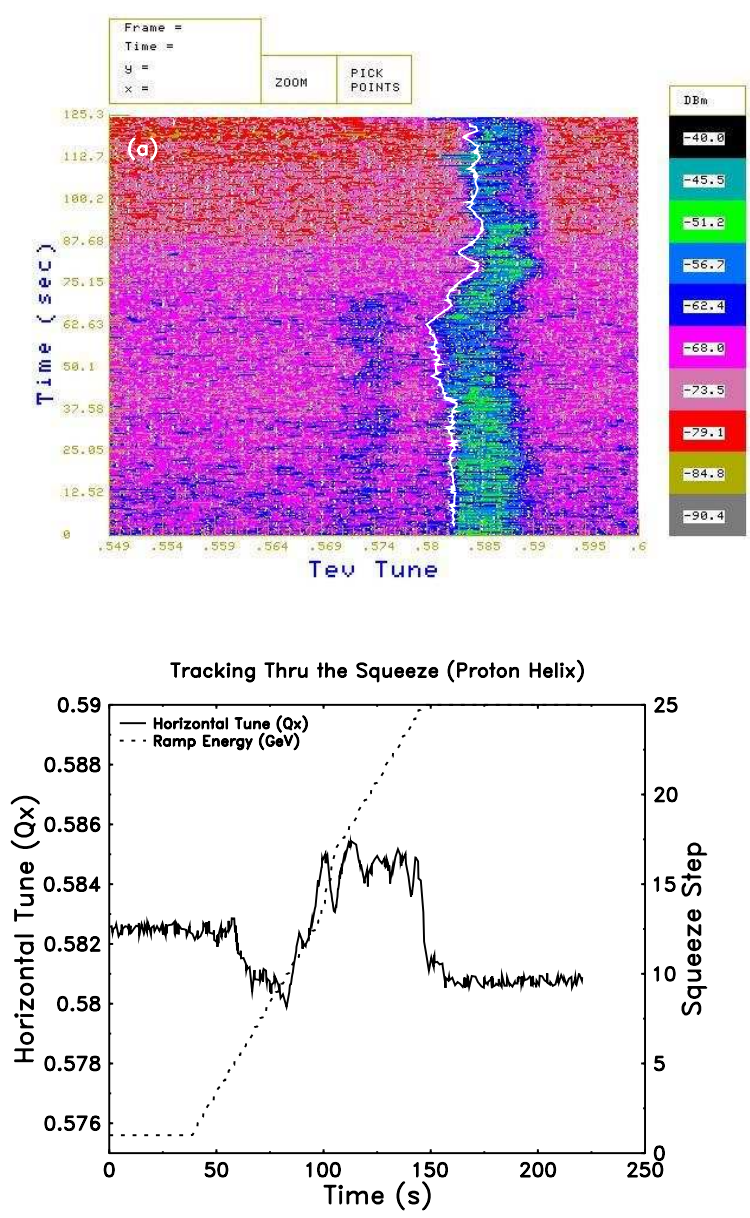

Figure 40 Tracking using continuous PLL through the squeeze on the proton helix with one bunch of coalesced protons. Picture (a) is the proton helix waterfall spectrum of store number 3699 without the PLL going through the squeeze. Superimposed on this is the PLL locked frequency from another store. Finally, the bottom picture is the same PLL tune data plotted against the Tevatron squeeze. 

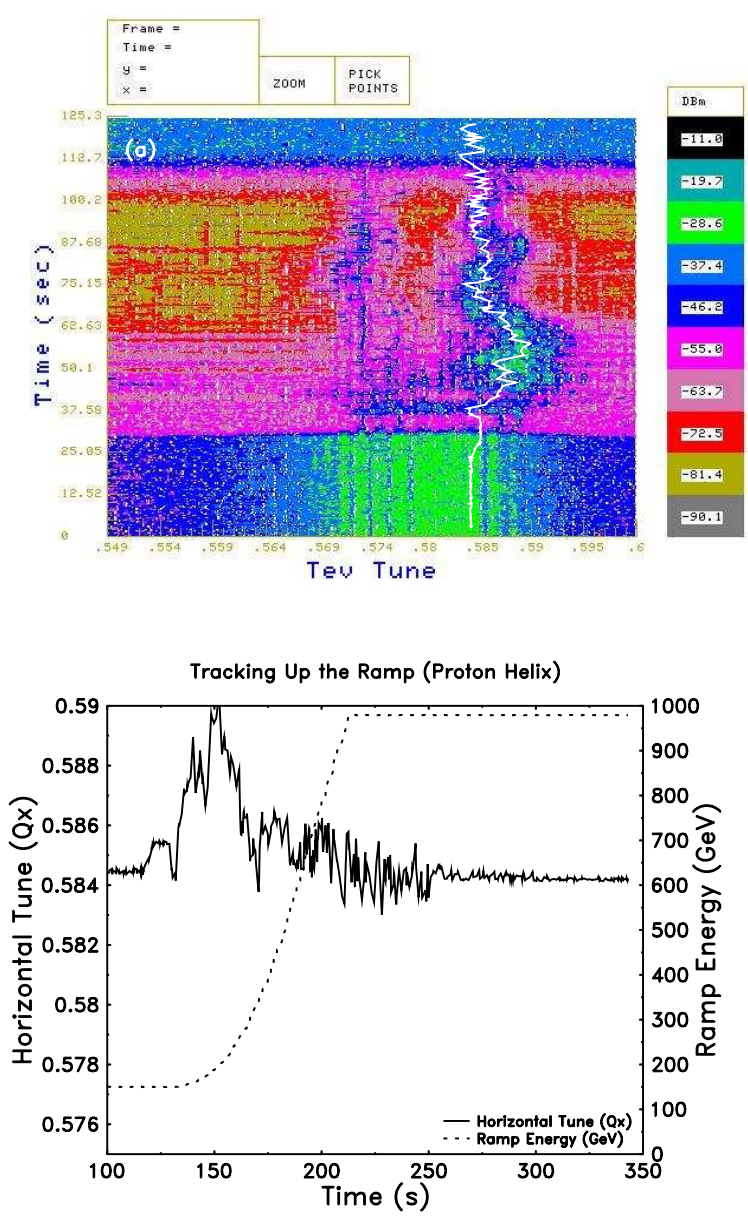

Figure 41 Tracking using pulsed PLL up the ramp on the proton helix with one bunch of coalesced protons. Picture (a) is the proton helix waterfall spectrum of store number 3699 without the PLL going up the ramp. Superimposed on this is the PLL locked frequency data from another store. Finally, the bottom picture is the same PLL tune data plotted against the Tevatron energy ramp. 

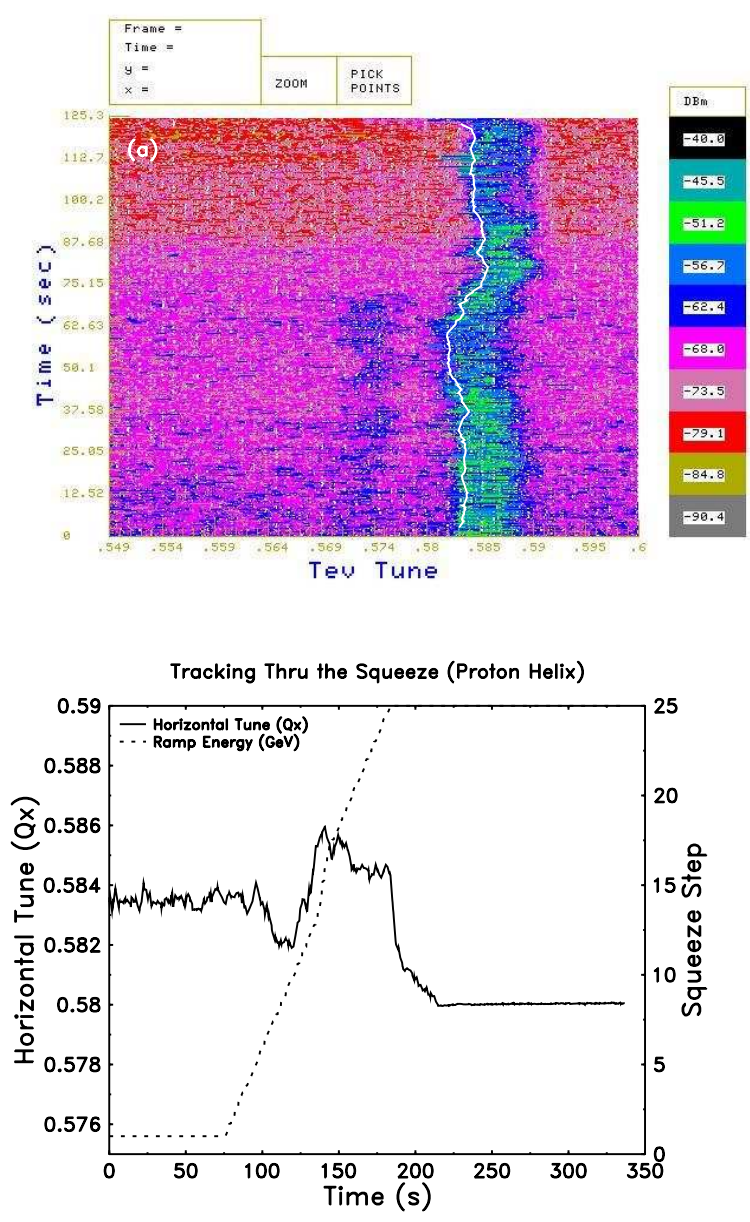

Figure 42 Tracking using pulsed PLL through the squeeze on the proton helix with one bunch of coalesced protons. Picture (a) is the proton helix waterfall spectrum of store number 3699 without the PLL going through the squeeze. Superimposed on this is the PLL locked frequency data from another store. Finally, the bottom picture is the same PLL tune data plotted against the Tevatron squeeze. 


\section{EMITTANCE GROWTH}

The emittance growth of 1 bunch of coalesced protons on the proton helix is measured using flying wires at $150 \mathrm{GeV}$. After injecting this bunch into the Tevatron, the horizontal emittance is measured to be at $8.1 \pi \mathrm{mm} \cdot \mathrm{mrad}$. After 10 minutes, the horizontal emittance grew to $9.86 \pi \mathrm{mm} \cdot \mathrm{mrad}$ without any kicking from the PLL. We then turn the PLL on with the kicker set to $180 \mathrm{~mW}$ for 10 minutes. The horizontal emittance after 10 minutes is $10.6 \pi \mathrm{mm} \cdot \mathrm{mrad}$. This means that the emittance has grown by $0.74 \pi \mathrm{mm} \cdot \mathrm{mrad}$ which is smaller than the first 10 minutes without the kicker. See Figure 43. Although we have not proved that there is no emittance growth due to the PLL kicker, we have at least shown that the emittance does not grow by a factor of 2 during this time. The vertical emittance did not grow appreciably during the 10 minutes of kicking. Table 3 summarizes the results.

\begin{tabular}{c|c|c|l}
\hline \multicolumn{4}{c}{ Table 3. Emittance Growth } \\
\hline $\boldsymbol{\epsilon}_{\boldsymbol{x}}$ start & $\boldsymbol{\epsilon}_{\boldsymbol{x}}$ stop & time (min) & \multicolumn{1}{c}{ Comments } \\
8.10 & 9.86 & 10 & kicker off \\
9.86 & 10.6 & 10 & kicker on and PLL locked \\
\hline \hline
\end{tabular}




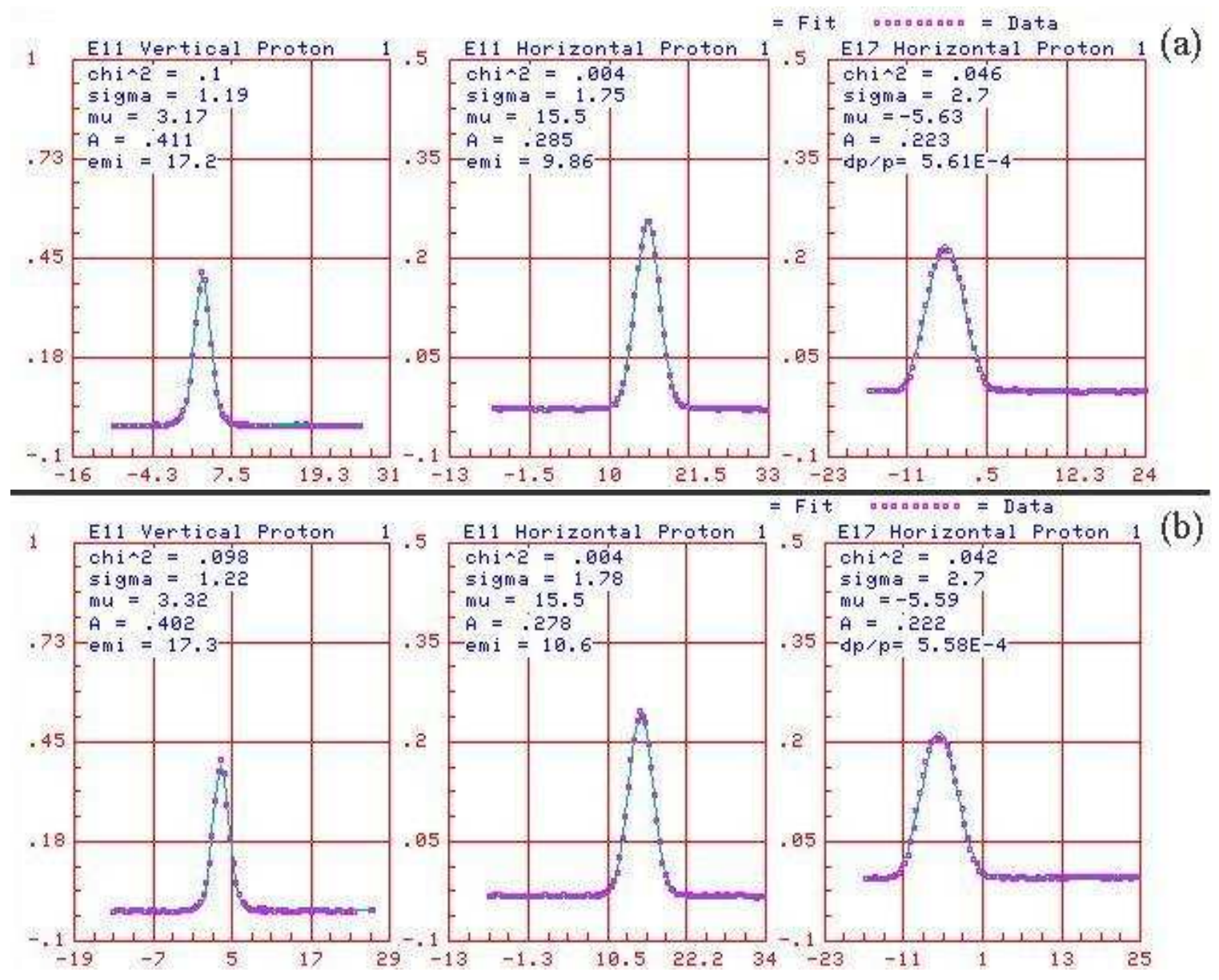

Figure 43 The emittance of one coalesced bunch on the helix before being kicked continuously by the PLL is shown in (a). The emittance after 10 minutes of continuously kicked by the PLL is shown in (b). 
Epilogue 


\section{PLANS FOR 2005}

The Tevatron is shutdown from August 2004 to November 2004. Tune tracker studies will probably resume in January 2005 at the earliest. The plan is to

(i) Implement kicker feedback.

(ii) Implement phase compensation.

(iii) Test out multitone PLL.

(iv) Make a copy of the tune tracker for the vertical plane.

$(v)$ Start work on chromaticity measurement which critically depends on the success of the PLL.

\section{CONCLUSION}

After less than a year of work, the tune tracker system at Fermilab is in good shape to make more progress next year. We have found that the continuous and pulsed methods of excitation do work up the ramp and squeeze. Although there is no compelling reason to use pulsed excitation up the ramp for one plane only, there may be an argument for using it when we have both planes working if coupling turns out to be a problem. We can also think of other scenarios where we can use the pulsed PLL to find the centre of the betatron tune and then use continuous PLL to track it. However, these speculations of how to use both methods will have to wait for the reality of beam next year. From our successful experience up to this point, we believe that the concepts described in this paper can also be used at the LHC. 


\section{ACKNOWLEDGEMENTS}

The author would like to thank the following people:

(i) J. Marriner for suggesting that I take on this project.

(ii) P. Cameron for giving me useful ideas from his experience with the BNL tune tracker PLL.

(iii) K. Koch for drawing up the daughtercard schematic from my chicken scratchings and especially his steady hand when soldering all the surface mount components while fabricating it.

(iv) B. Fellenz for distributing the $21.4 \mathrm{MHz}$ local RF and the Schottky signals to our PLL.

$(v)$ D. Peterson for providing the "fake beam" simulator using the technique of commutating filters.

(vi) J. Steimel for useful discussions.

(vii) The Tevatron group for their support in getting machine studies time for commissioning the tune tracker. 
Appendices 


\section{APPENDIX I}

The goal for building a fake beam frequency response is to relieve us from always asking for machine studies time in order to test out the PLL. We wanted to have a circuit that is simple and has the capability of changing its resonance frequency quickly and easily. This request was quickly fulfilled by a circuit built by D. Peterson called a commutating filter which is a non linear circuit whose frequency response is a comb filter like structure. The positions of the tooths of the comb in frequency space are controlled by an external clock and thus by changing the frequency of this clock, we can easily change where the teeth are. In this circuit, they are at multiples of (clock frequency)/8. The use of this filter is immediately obvious: the lowest frequency tooth in the comb filter can be thought of as the betatron tune. The position of the tooth can be easily changed by changing the clock frequency, thus mimicking frequency changes in the betatron tune. At this time, the fake beam response circuit is limited to representing a betatron tune with zero chromaticity, i.e. no synchrotron lines. An upgraded design is now being built by J. Ning and C.Y. Tan which will be able to mimic synchrotron lines and chromaticity. (Estimated completion Spring 2005).

In the next two sections, we will calculate the frequency response and the bandwidth of the commutating filter and also show the measured frequency response of the actual circuit.

\section{Commutating Filter}

The commutating filter is shown in Figure 44. It consists of a resistor $R$ and $N$ capacitors $C$. Each one of these capacitors is switched into the circuit with a period $T_{s}$ and is left in the circuit for $T_{s} / N$. See Figure 45 . This implies that the switch frequency 


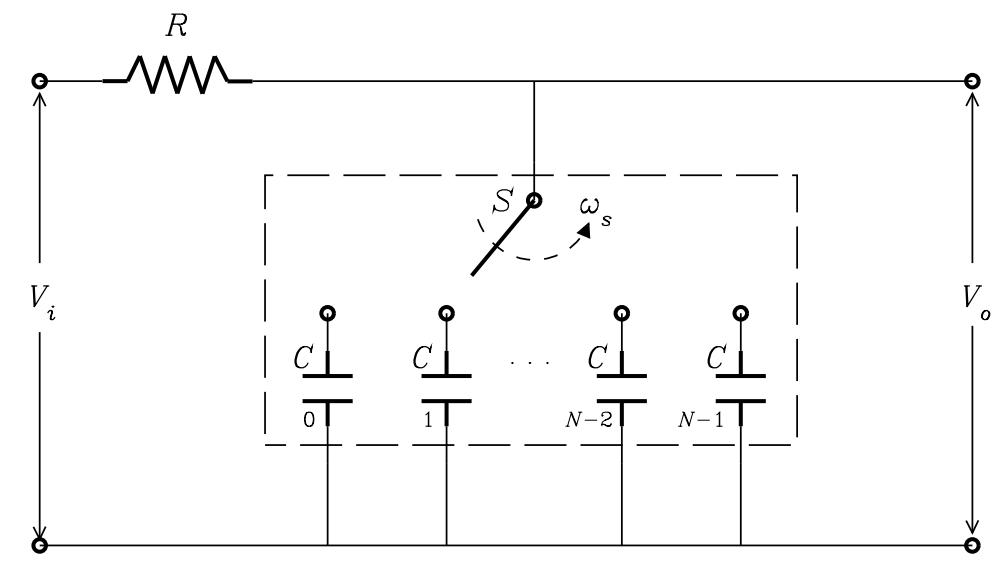

Figure 44 The commutating filter consists of a resistor $R$ and $N$ capacitors $C$ numbered from $0,1, \ldots, N-1$. The switch $S$ rotates at frequency $\omega_{s}$ and connects each capacitor $C$ for a time period of $T_{s} / N$

$\omega_{s}=2 \pi N / T_{s}$.

Let us look at one of the circuits with $R$ and the $n$th capacitor $C$. See Figure 46 . We will calculate $v_{o}$ when $v_{i}=\tilde{v}_{i} e^{i \Omega t} .^{\dagger}$ The differential equation which relates $v_{o}$ to $v_{i}$ is

$$
\left.\begin{array}{c}
I R+\frac{Q}{C}=v_{i} \\
\quad \dot{Q} R+\frac{Q}{C}=v_{i}
\end{array}\right\}
$$

where $I$ is the current in the circuit, $Q$ the charge stored in the capacitor. The initial condition $Q\left(t_{k}\right)=Q_{k}$.

(82) is easily solved for $Q$ and yields

$$
Q(t)=\left(Q_{k}-\frac{\tilde{v}_{i} C e^{i \Omega t_{k}}}{1+i \Omega R C}\right) e^{t_{k} / R C} e^{-t / R C}+\frac{\tilde{v}_{i} C e^{i \Omega t}}{1+i \Omega R C}
$$

$\dagger$ The choice of $e^{i \Omega t}$ rather than $e^{-i \Omega t}$ is because we will be using the engineer's definition of the Fourier transform rather than the physicist's definition. 


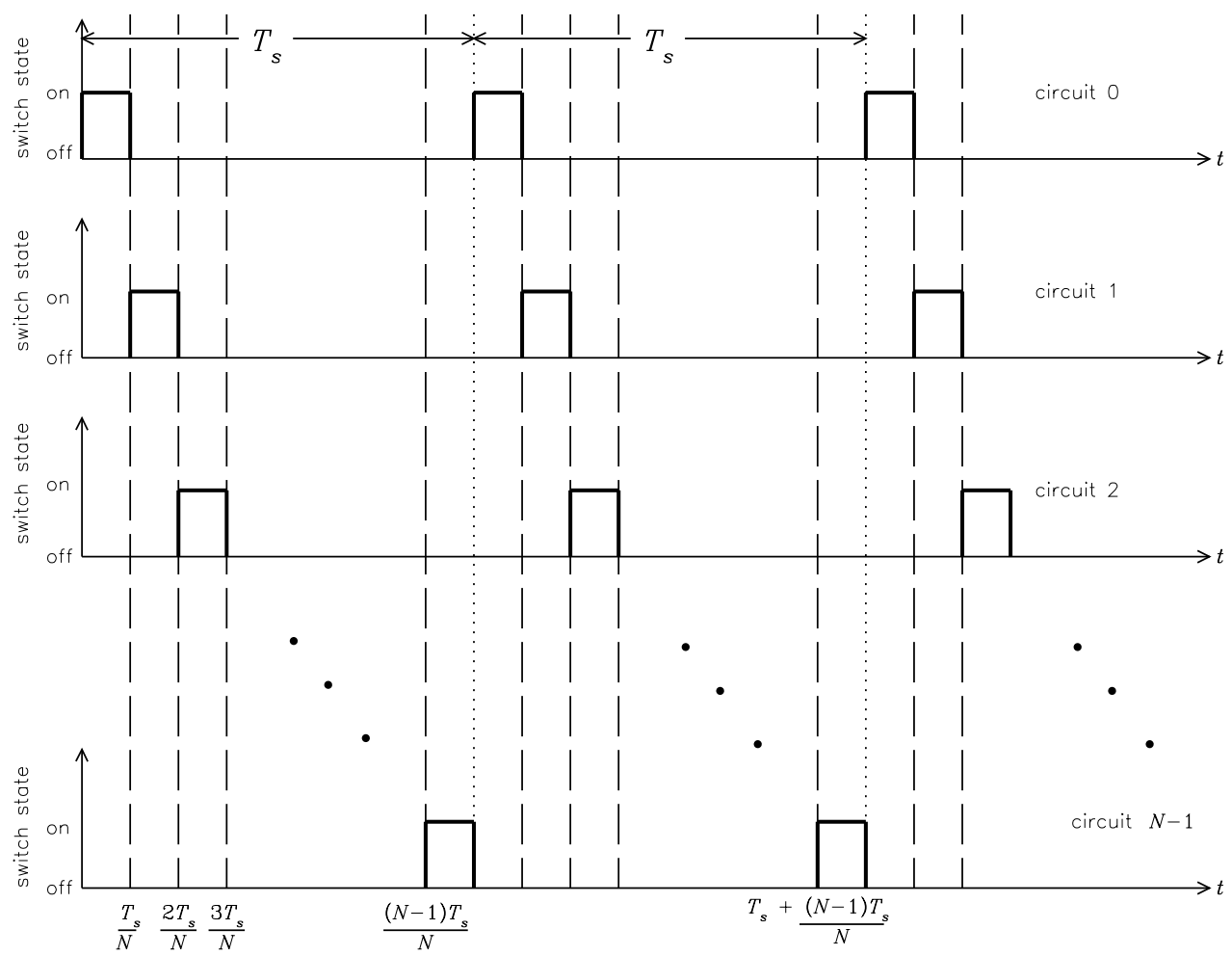

Figure 45 Each capacitor $C$ is connected to $R$ for a time period $T_{s} / N$ and the time between each "on" period is $T_{s}$.

Next, let us look at how the charge $Q$ on the capacitor evolves as a function of time $t$. Looking at Figure 46, let us assume that at $t=n T_{s} / N$, the initial charge is chosen so that

$$
Q(k=0)=\frac{C \tilde{v}_{i} e^{i(n+1) \Omega T_{s} / N}}{1+i \Omega R C} \quad \text { at } t=(n+1) T_{s} / N .
$$

We can do this because we will be looking at the asymptotic value of $Q$. Continuing on, for $k=1$, the initial charge is given by $Q(k=0)$ and so at $t=T_{s}+(n+1) T_{s} / N$

$$
Q(k=1)=\frac{C \tilde{v}_{i}}{1+i \Omega R C}\left[\left(e^{i(n+1) \Omega T_{s} / N}-1\right) e^{-T_{s} / N R C}+e^{i \Omega T_{s}\left(1+\frac{n+1}{N}\right)}\right]
$$

And in general at $t=k T_{s}+(n+1) T_{s} / N$

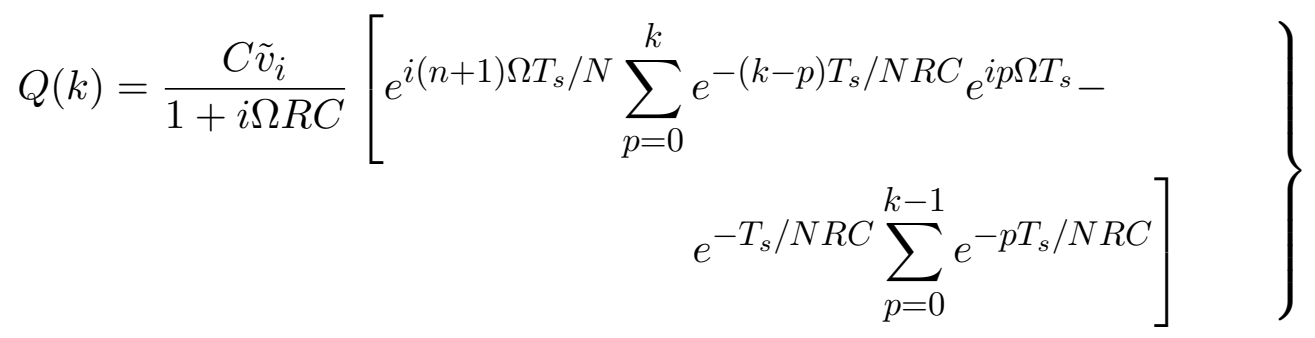



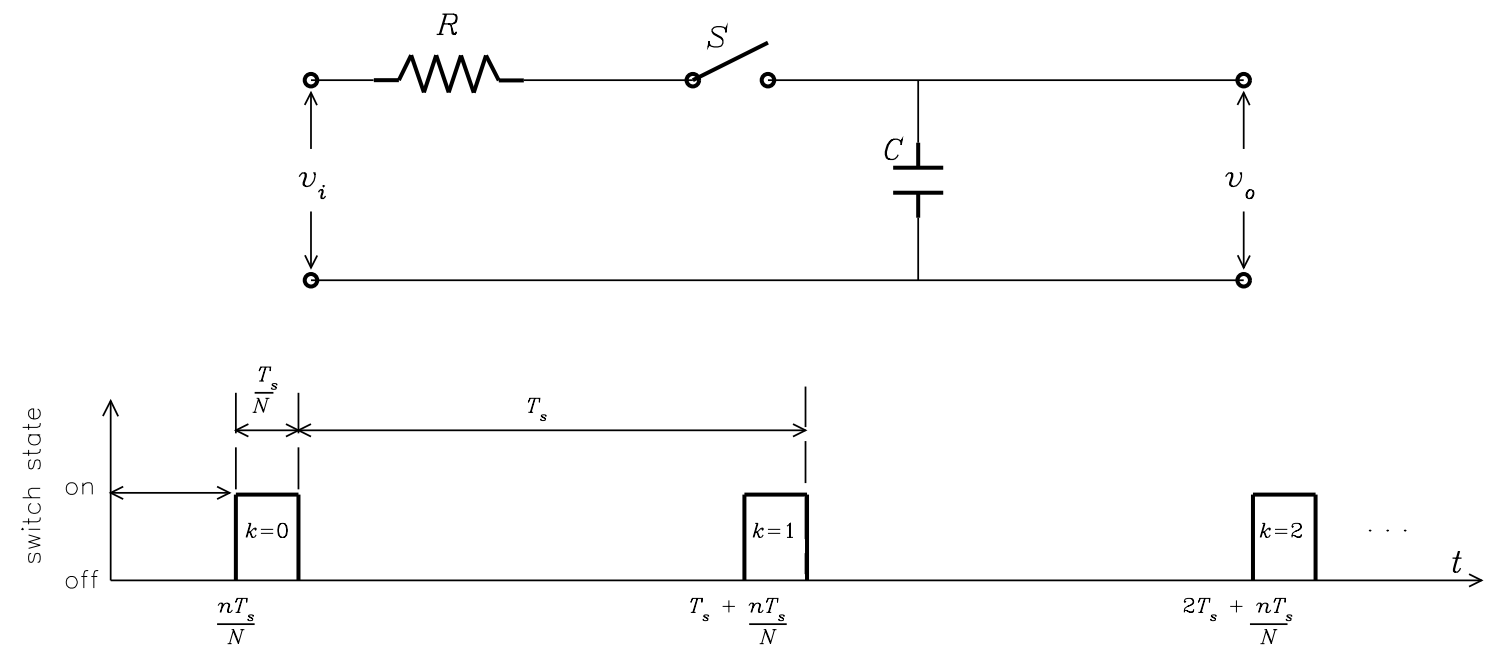

Figure 46 Looking at the $n$th $R C$ circuit, the switch is turned on every $k T_{s}+n T_{s} / N$ and stays on for $T_{s} / N$.

For large $k$, we can use the formula for the infinite geometric sum

$$
\begin{aligned}
Q(k, n) & =\frac{C \tilde{v}_{i} e^{i\left(k+\frac{n+1}{N}\right) \Omega T_{s}}}{(1+i \Omega R C)\left(1-e^{-T_{s} / N R C} e^{-i \Omega T_{s}}\right)}-\frac{C \tilde{v}_{i} e^{-T_{s} / N R C}}{(1+i \Omega R C)\left(1-e^{-T_{s} / N R C}\right)} \\
& \equiv Q(\Omega) e^{i\left(k+\frac{n+1}{N}\right) \Omega T_{s}}+\text { DC offset independent of } k \text { and } n
\end{aligned}
$$

where we have used $\sum_{p=0}^{k} e^{-(k-p) T_{s} / N R C} e^{i p \Omega T_{s}}=\sum_{p=0}^{k} e^{-p T_{s} / N R C} e^{i(k-p) \Omega T_{s}}$. (87) tells us that after a long time, for a fixed $\Omega$, the charge on the right edge of the "on" interval of the $n$th circuit is dependent on the sinusoidal term. The DC term is independent of $k$ and $n$ and is the same in each "on" interval. Since the DC term is constant, we will ignore it for the rest of the analysis.

Now, let us put in all the capacitors and make the approximation that the charge in each "on" interval is constant and is given by (87). See Figure 47. Note that in this 
approximation $Q(k, n)$ is the charge in the "on" interval numbered $(k, n+1)$ and the first "on" interval has zero charge. We do this so that our result is causal.
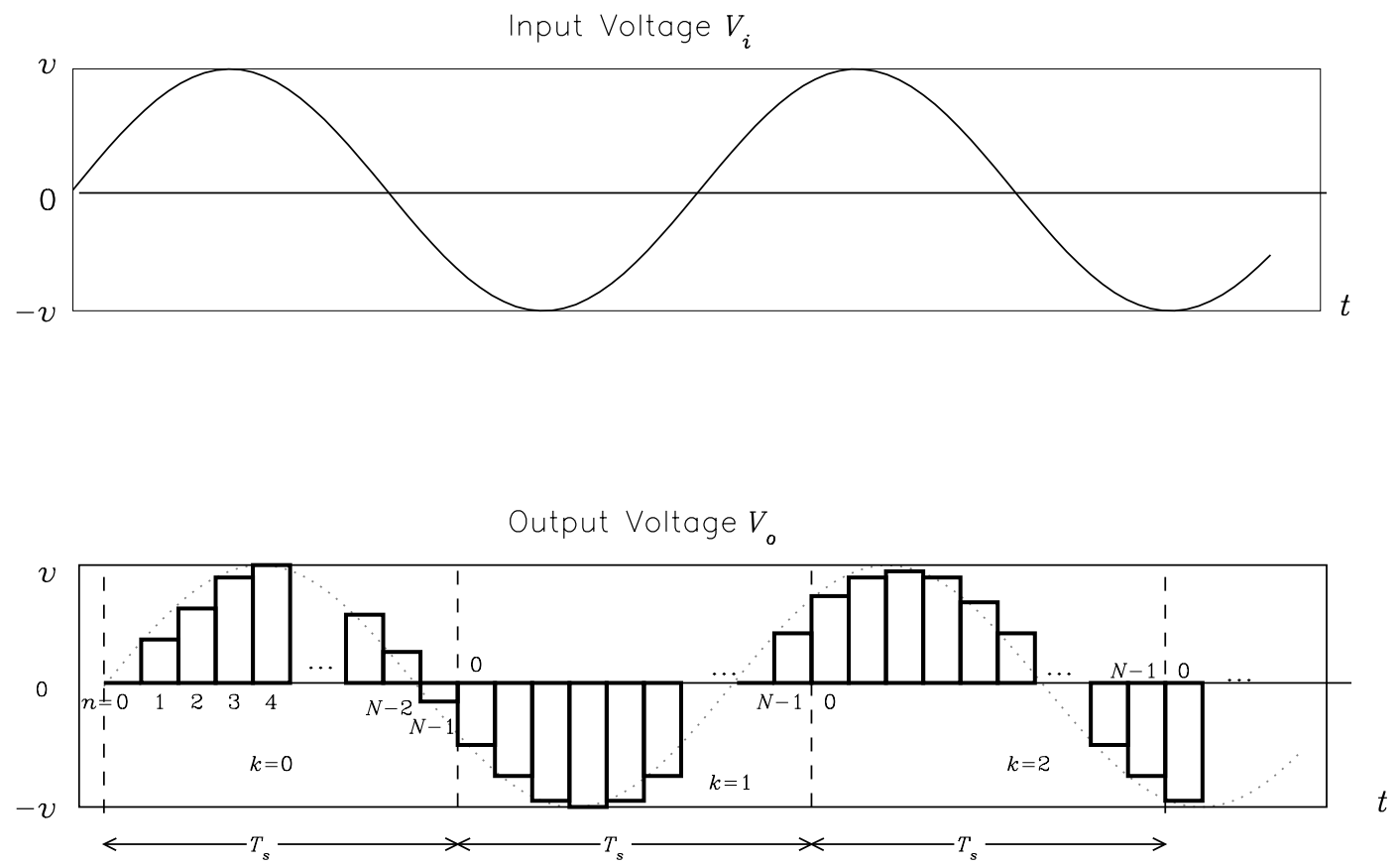

Figure 47 In this example we have a sinusoidal input $V_{i}$ and at the output using our approximation — we have boxes which also look sinusoidal.

We are going to renumber the counters $(k, n)$ in $(87)$ to $\ell$, i.e.

$$
Q(\Omega) e^{i\left(k+\frac{n+1}{N}\right) \Omega T_{s}} \rightarrow Q(\Omega) e^{i(\ell+1) \Omega T_{s} / N} \quad\left\{\begin{array}{l}
k=0, \ldots, \infty \text { and } n=0, \ldots, N-1 \\
\ell=0, \ldots, \infty
\end{array}\right.
$$

Therefore, the output $V_{O}$ is

$$
V_{o}(t)=\tilde{V}_{i}(\Omega) \sum_{\ell=0}^{\infty}\left[u\left(t-\frac{(\ell+1) T_{s}}{N}\right)-u\left(t-\frac{(\ell+2) T_{s}}{N}\right)\right] e^{i(\ell+1) \Omega T_{s} / N}
$$

where $V_{i}(\Omega)=Q(\Omega) / C$ and $u(t)$ is the step function. 
We need the component of $V_{o}(t)$ at $\Omega$ in order to calculate the frequency response of the commutating filter. The Fourier transform is

$$
\begin{aligned}
\tilde{V}_{o}(\Omega) & =\lim _{M \rightarrow \infty} \tilde{V}_{i}(\Omega) \sum_{\ell=0}^{M} e^{i(\ell+1) \Omega T_{s} / N} \times \\
& \int_{-\infty}^{\infty} d t\left[u\left(t-\frac{(\ell+1) T_{s}}{N}\right)-u\left(t-\frac{(\ell+2) T_{s}}{N}\right)\right] e^{-i \Omega t} \\
& =\lim _{M \rightarrow \infty} \frac{i \tilde{V}_{i}(\Omega)}{\Omega} \sum_{\ell=0}^{M}\left(e^{-i \Omega T_{s} / N}-1\right) \\
& =\tilde{V}_{i}(\Omega) e^{-i \Omega T_{s} / 2 N} \frac{\sin \left(\frac{\Omega T_{s}}{2 N}\right)}{\frac{\Omega}{2}} \times \lim _{M \rightarrow \infty}(M+1)
\end{aligned}
$$

The infinite sum comes from evaluating $\tilde{V}_{O}$ at $\Omega$. This should be compared to calculating the Fourier transform of $e^{i \Omega t}$ which gives us a Dirac delta function $\delta(\omega-\Omega)$. And when evaluated at $\omega=\Omega$ gives infinity.

The Fourier transform of the excitation at $\Omega$ which goes from $t=0$ to $t=(M+1) T_{s} / N$ with $M \rightarrow \infty$ is

$$
\begin{aligned}
\tilde{V}_{i}(\Omega) & =\lim _{\substack{M \rightarrow \infty \\
\omega \rightarrow \Omega}} \tilde{v}_{i} \int_{-\infty}^{\infty} d t\left[u(t)-u\left(t-\frac{(M+1) T_{s}}{N}\right)\right] e^{i(\Omega-\omega) t} \\
& =\lim _{M \rightarrow \infty} \tilde{v}_{i}(M+1) T_{s} / N
\end{aligned}
$$

which means that the frequency response is easily derived from (90) and (91) and is independent of $M$

$$
\begin{aligned}
H(\Omega)=\frac{\tilde{V}_{o}(\Omega)}{\tilde{V}_{i}(\Omega)} & =\frac{e^{-i \Omega T_{s} / 2 N}}{1+i \Omega R C} \times \frac{1}{1-e^{-T_{s} / N R C_{e}-i \Omega T_{s}}} \times \frac{\sin \left(\frac{\Omega T_{s}}{2 N}\right)}{\frac{\Omega T_{s}}{2 N}} \\
& =\text { phase shift } \times \text { resonance } \times \text { rectangular sample }
\end{aligned}
$$

Examining the terms in (92) gives us a phase shift which depends on $\Omega, R$, and $C$. The sinc term comes from the approximation that the voltage is constant in each "on" interval. The resonance term is more interesting: it is maximum when $e^{-i \Omega T_{s}}$ is completely real 

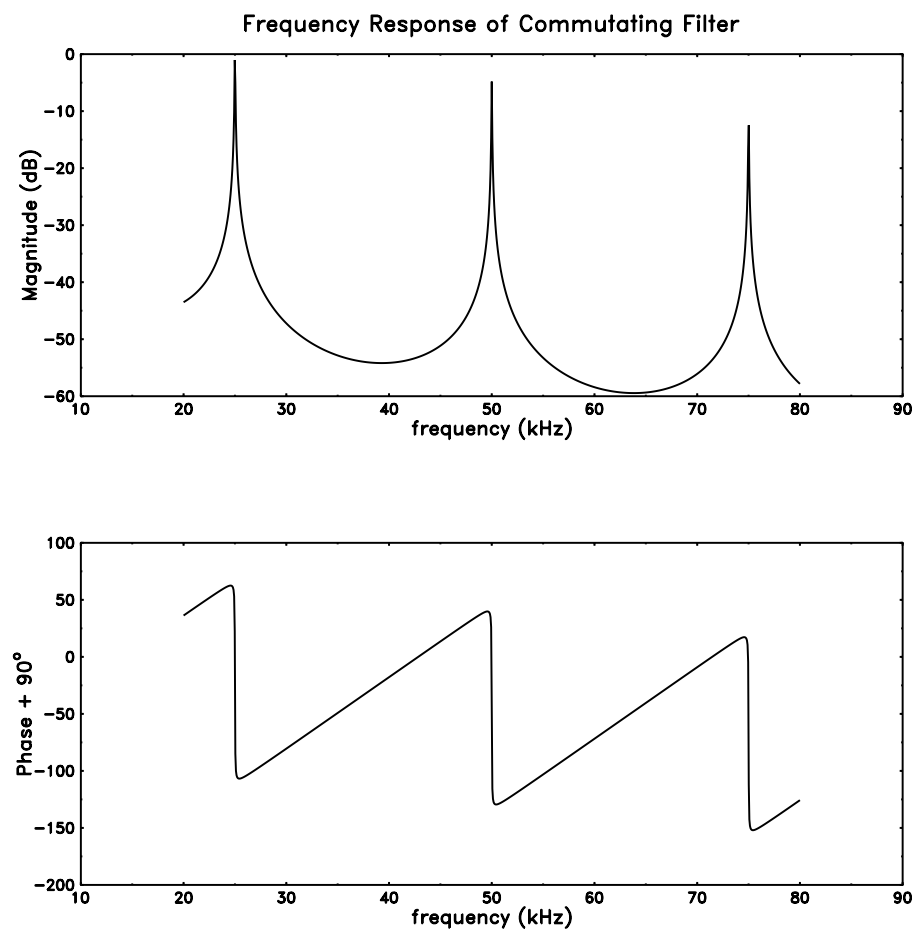

Figure 48 The frequency response $H(\Omega)$ of the commutating filter. We have added $90^{\circ}$ to the phase so that we do not have the wrap around effect at $\pm 180^{\circ}$.

and positive, this means that

$$
\left.\begin{array}{rl}
T_{s} & =N\left(\frac{2 \pi}{\omega_{s}}\right) \\
\therefore \quad \Omega T_{s} & =\Omega \times N\left(\frac{2 \pi}{\omega_{s}}\right)=2 k \pi \quad k \in \mathbb{Z} \quad
\end{array}\right\}
$$

so that the peaks of the resonance $\Omega_{\text {res }}$ are at

$$
\Omega_{\mathrm{res}}=k\left(\frac{\omega_{s}}{N}\right) \quad k \in \mathbb{Z}
$$

For example if the sampling frequency is $200 \mathrm{kHz}$ and $N=8$, then $f_{\text {res }}=25,50, \ldots \mathrm{kHz}$.

The plot of the frequency response $H(\Omega)$ is shown in Figure 48 for the special case of $N=8, R=10 \mathrm{k} \Omega, C=0.1 \mu \mathrm{F}, \omega_{s}=2 \pi \times 200 \times 10^{3} \mathrm{~s}^{-1}$. The plot of the frequency response of an actual commutating filter is shown in Figure 49. 

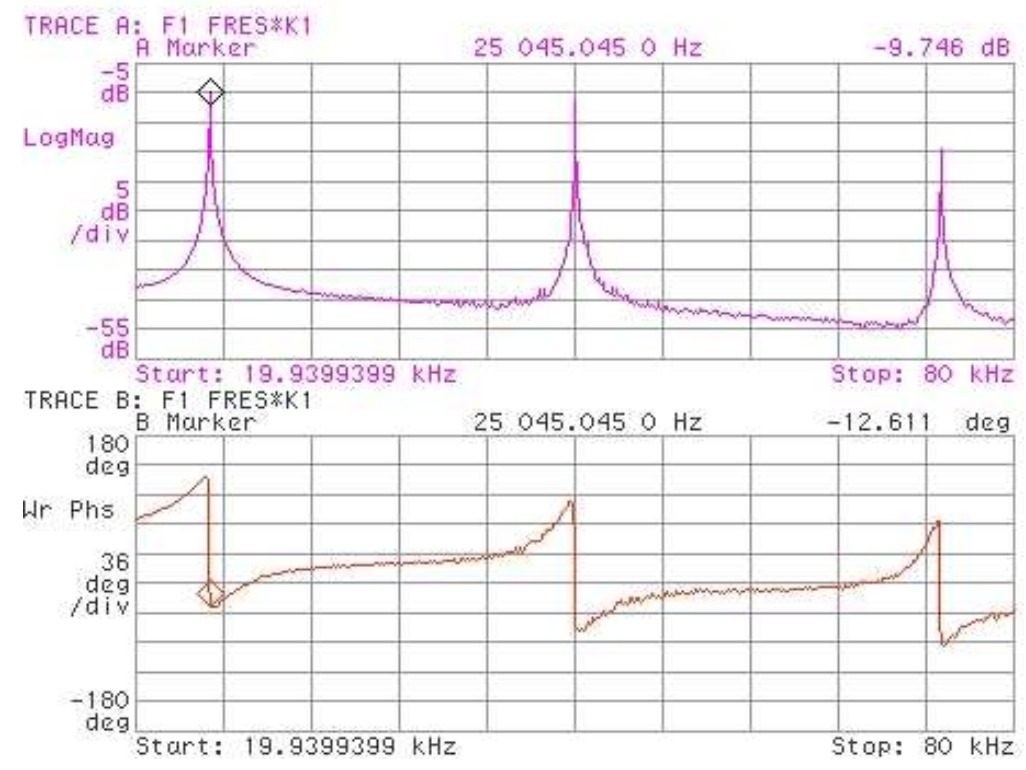

Figure 49 The frequency response of the commutating filter measured with a VSA. The phase is also shifted by $90^{\circ}$.

\section{Bandwidth}

The bandwidth of the resonances can be calculated from just the resonance term. The power at resonance is

$$
\tilde{V}_{\text {res }}^{2}=\frac{1}{1-e^{-T_{s} / N R C}}
$$

We need to solve for $\Omega$ at $V_{2}^{2} / 2$ i.e.

$$
\frac{1}{1-e^{-T_{s} / N R C} e^{-i \Omega T_{s}}} \times \text { complex conjugate }=\frac{1}{2\left(1-e^{-T_{s} / N R C}\right)}
$$

To make things simple, we will assume that $T_{s} \ll N R C$. For example for $N=8, R=$ $10 \mathrm{k} \Omega, C=0.1 \mu \mathrm{F}, \omega_{s}=2 \pi \times 200 \times 10^{3} \mathrm{~s}^{-1}$ which implies that $T_{s}=40 \mu \mathrm{s}$ and $N R C=8 \mathrm{~ms}$ which satisfies the assumption.

Inverting and expanding (96) we find that

$$
\cos \Omega T_{s}=1-\frac{1}{2}\left(\frac{T_{s}}{N R C}\right)^{2}+\mathrm{O}\left(\frac{T_{s}}{N R C}\right)^{3}
$$


where we have made the approximation that $e^{-T_{s} / N R C} \approx 1-T_{s} / N R C$. Solving for $\Omega$ from (97)

$$
\begin{aligned}
\Omega & =\frac{1}{T_{s}} \cos ^{-1}\left[1-\frac{1}{2}\left(\frac{T_{s}}{N R C}\right)^{2}\right] \\
& \approx \frac{1}{N R C}+k \Omega_{\mathrm{res}} \quad k \in \mathbb{Z} \\
& \equiv \Delta \Omega_{1 / 2}+k \Omega_{\mathrm{res}} \quad k \in \mathbb{Z}
\end{aligned}
$$

The bandwidth of each resonance is given by $2 \Delta \Omega_{1 / 2}$ and thus

$$
\Delta \Omega_{\mathrm{bw}}=\frac{2}{N R C} \Rightarrow f_{\mathrm{bw}}=\frac{\Delta \Omega_{\mathrm{bw}}}{2 \pi}=\frac{1}{\pi N R C}
$$




\section{APPENDIX II}

\begin{tabular}{c|c|c|c}
\multicolumn{5}{c}{ Table 4 . Hilbert Transformer Filter Coefficients } \\
\hline $\boldsymbol{i}$ & coefficient $[\boldsymbol{i}]$ & $\boldsymbol{i}$ & coefficient $[\boldsymbol{i}]$ \\
0 & 0.0124427 & 50 & -0.0124427 \\
1 & 0.0000000 & 49 & 0.0000000 \\
2 & 0.00900775 & 48 & -0.00900775 \\
3 & 0.0000000 & 47 & 0.0000000 \\
4 & 0.0122483 & 46 & -0.0122483 \\
5 & 0.0000000 & 45 & 0.0000000 \\
6 & 0.0162677 & 44 & -0.0162677 \\
7 & 0.0000000 & 43 & 0.0000000 \\
8 & 0.0212626 & 42 & -0.0212626 \\
9 & 0.0000000 & 41 & 0.0000000 \\
10 & 0.0275401 & 40 & -0.0275401 \\
11 & 0.0000000 & 39 & 0.0000000 \\
12 & 0.0355514 & 38 & -0.0355514 \\
13 & 0.0000000 & 37 & 0.0000000 \\
14 & 0.0461607 & 36 & -0.0461607 \\
15 & 0.0000000 & 35 & 0.0000000 \\
16 & 0.0608791 & 34 & -0.0608791 \\
17 & 0.0000000 & 33 & 0.0000000 \\
18 & 0.0831083 & 32 & -0.0831083 \\
19 & 0.0000000 & 31 & 0.0000000 \\
20 & 0.121635 & 30 & -0.121635 \\
21 & 0.0000000 & 29 & 0.0000000 \\
22 & 0.208752 & 28 & -0.208752 \\
23 & 0.0000000 & 27 & 0.0000000 \\
24 & 0.635464 & 26 & -0.635464 \\
25 & 0.0000000 & & \\
\hline \hline & & & \\
\hline
\end{tabular}




\section{REFERENCES}

[1] P. Cameron et al, Tune Feedback at RHIC, Proceedings of the Particle Accelerator Conference, 2001.

[2] P. Cameron et al, PLL Tune Measurement during RHIC 2001, Proceedings of the European Particle Accelerator Conference, 1855-1857, 2002.

[3] P. Cameron et al, RHIC Third Generation PLL Tune System, Proceedings of the Particle Accelerator Conference, 524-526, 2003.

[4] J.G. Proakis, Digital Signal Processing: Principles, Applications and Algorithms, Prentice-Hall, 1995.

[5] R.E. Best, Phase-Locked Loops Design, Simulation and Applications 4th Edition, McGraw-Hill, 1999.

[6] AD8361: LF to 2.5 GHz TruPwr Detector datasheet, Analog Devices.

[7] D. Martin et al, A Resonant Beam Detector for Tevatron Tune Monitoring, Accelerator Science and Technology Proceedings, 3, 1486-1488, 1989.

[8] D. Whitlow, Design and Operation of Automatic Gain Control Loops for Receivers in Modern Communications Systems, Analog Devices.

[9] R. Pasquinelli, Private Communication, 2004.

[10] M. Lauterbach and W. Swirnow, Using a Digital Oscilloscope for Signal Analysis including a Practical Example of PLL Characterization, LeCroy Corporation. 